\title{
REPRESENTATIVE HYDRAULIC CONDUCTIVITIES IN SATURATED GROUNDWATER FLOW
}

\author{
Xavier Sanchez-Vila, ${ }^{1}$ Alberto Guadagnini, ${ }^{2}$ and Jesus Carrera ${ }^{1}$ \\ Received 24 March 2005; revised 5 January 2006; accepted 5 May 2006; published 23 September 2006.
}

[1] Heterogeneity is the single most salient feature of hydrogeology. An enormous amount of work has been devoted during the last 30 years to addressing this issue. Our objective is to synthesize and to offer a critical appraisal of results related to the problem of finding representative hydraulic conductivities. By representative hydraulic conductivity we mean a parameter controlling the average behavior of groundwater flow within an aquifer at a given scale. Three related concepts are defined: effective hydraulic conductivity, which relates the ensemble averages of flux and head gradient; equivalent conductivity, which relates the spatial averages of flux and head gradient within a given volume of an aquifer; and interpreted conductivity, which is the one derived from interpretation of field data. Most theoretical results are related to effective conductivity, and their application to real world scenarios relies on ergodic assumptions. Fortunately, a number of results are available suggesting that conventional hydraulic test interpretations yield (interpreted) hydraulic conductivity values that can be closely linked to equivalent and/or effective hydraulic conductivities. Complex spatial distributions of geologic hydrofacies and flow conditions have a strong impact upon the existence and the actual values of representative parameters. Therefore it is not surprising that a large body of literature provides particular solutions for simplified boundary conditions and geological settings, which are, nevertheless, useful for many practical applications. Still, frequent observations of scale effects imply that efforts should be directed at characterizing well-connected stochastic random fields and at evaluating the corresponding representative hydraulic conductivities.

Citation: Sanchez-Vila, X., A. Guadagnini, and J. Carrera (2006), Representative hydraulic conductivities in saturated groundwater flow, Rev. Geophys., 44, RG3002, doi:10.1029/2005RG000169.

\section{INTRODUCTION}

\subsection{Significance of Hydraulic Conductivity to Earth Sciences}

[2] The concept of hydraulic conductivity dates back to the middle nineteenth century, when Darcy [1856] performed experiments to show that fluid displacement in porous materials is governed by a linear relationship between velocity and hydraulic gradient. Dupuit [1863] noticed the parallelism with heat conduction and, being heavily influenced by the work of Fourier [1822], expanded the work of Darcy, providing the mathematical framework that led to the generalized form of Darcy's law as it is used nowadays:

$$
\mathbf{q}(\mathbf{x})=-\mathbf{K}(\mathbf{x}) \nabla \mathbf{h}(\mathbf{x}),
$$

where $\mathbf{x}$ is a vector of space coordinates, $\mathbf{q}$ is a seepage velocity vector, $\mathrm{h}$ is hydraulic head, and $\mathbf{K}$ is the hydraulic

\footnotetext{
${ }^{1}$ Department of Geotechnical Engineering and Geosciences, Technical University of Catalonia, Barcelona, Spain.

${ }^{2}$ Dipartimento di Ingegneria Idraulica, Ambientale, Infrastrutture Viarie, Rilevamento, Politecnico di Milano, Milan, Italy.
}

conductivity tensor. This tensor can be written as $\mathbf{K}=\mathbf{k} \rho \mathrm{g} / \mu$, where $\mathbf{k}$ is the intrinsic permeability tensor, $\rho$ is fluid density, $\mathrm{g}$ is the acceleration of gravity, and $\mu$ is viscosity. That is, $\mathbf{K}$ depends on the properties of the medium through $\mathbf{k}$ (the larger and better connected the pores, the larger $\mathbf{k}$ is) and the fluid through $\rho$ and $\mu$. Hydraulic conductivity is a key parameter in several branches of Earth sciences and engineering, notably hydrogeology, soil sciences, geotechnics, and petroleum engineering. Because of the broad spectrum of disciplines some nomenclature ambiguities have arisen. In civil engineering, hydraulic conductivity is often termed permeability, which is the way geologists and petroleum engineers term intrinsic permeability. Here we will use the term hydraulic conductivity.

[3] Darcy's law is an empirical law resulting from experimental evidence. However, it can be viewed from at least two other perspectives. Historically, early developments in groundwater flow arose from potential theory because hydraulic head is the energy per unit weight of water. Under slow laminar flow conditions, water flux is linearly proportional to head loss, which is another way of stating Darcy's law. This linearity between potential loss and flux is observed in many other phenomena, starting from Fourier's 
law, where thermal conductivity relates heat flux and temperature gradient. In fact, the publication of Fourier's [1822] book led to many laws equivalent to (1) [Narasimhan, 1999]: Ohm's law, which relates electricity current to the gradient of electrical potential through electrical conductivity; Fick's law, which relates diffusive fluxes of matter to the gradient of concentration through the molecular diffusion coefficient; Hooke's law that relates mechanical stress to the gradient of displacements through the elasticity modulus, etc. All these phenomena are historically and mathematically equivalent to water flux in porous media. As we will see, the singularity of hydraulic conductivity results from its large but structured variability.

[4] Darcy's law can also be viewed as an expression of Newton's second law: momentum conservation. For a given pore geometry and velocity field the force that the solid exerts over a Newtonian fluid is proportional to the flux. In the absence of gravity this force must be balanced by a pressure drop to maintain equilibrium. Therefore flux is proportional to pressure drop, which is yet another way of stating Darcy's law. As a consequence, Darcy's law can also be viewed as the porous medium version of Navier-Stokes equations.

[5] By controlling the direction and magnitude of flow, hydraulic conductivity plays a key role in many applica-
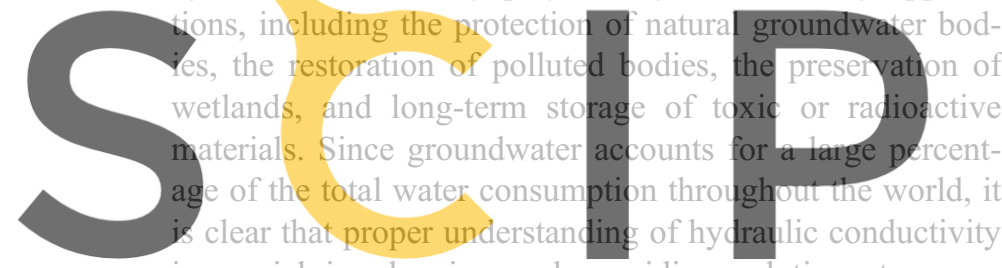

s crucial in planning and providing solutions to many environmental, economic, and societal challenges. Register for free at https//www.
1.2 . Motivation and Background

[6] To some extent the difficulties in properly understanding and identifying hydraulic conductivity can be traced to its almost intrinsic spatial variability. Over 30 years have passed since Freeze [1975] published a paper pointing to the effect of heterogeneity on groundwater flow across a column. While the paper had some limitations [Dagan, 1976; Gelhar et al., 1977], it brought to the attention of most hydrogeologists the importance of accounting for heterogeneity in hydrologic analysis. Actually, hydraulic conductivity $(\mathrm{K})$ is arguably one of the most variable parameters in the Earth sciences. Laboratory measurements of K span more than 12 orders of magnitude. Even in seemingly homogeneous aquifers, measured values of $\mathrm{K}$ may range over some orders of magnitude. This realization spurred a large amount of work on the problem of finding the effect of heterogeneity on flow and transport. Since hydrologic analyses of flow are largely based on the concept of hydraulic conductivity, an immediate question is whether the heterogeneous distribution can be substituted by a representative value, and, if so, what would such a value be. In short, one could ask two key questions: (1) Which are the actual values that can be considered representative? (2) How can one obtain them? These questions, in turn, generate some additional considerations, including whether representative values can actually be defined and how accurately can they be obtained on the basis of available data and information.

[7] The aim of this paper is precisely to address all of these questions. We will only review works dealing with representative hydraulic conductivities. Still, we must stress the large volume of literature produced in a number of disciplines with the aim of finding representative parameters in the theory of conductive media. Such disciplines would include electrical conductivity (see, e.g., Fokin [1996] for a review and Kaganova [2003]), thermal conductivity [e.g., Caldimi and Mahajan, 1999; Boomsma and Poulikakos, 2001], galvanomagnetic conductivity [e.g., Kaganova and Kaganov, 2004; Bergman and Stroud, 2000], and electronic conductivity [e.g., Du et al., 2004].

[8] Either a deterministic or a random model may be chosen to face real problems in hydrogeology. As discussed by Dagan [1997], the choice is always affected by how uncertainty is viewed. Deterministic approaches are based on viewing parameters as unique, but uncertain, local quantities at some given scale. Stochastic approaches are based on viewing reality as one among the ensemble of possible spatial distributions (realizations) of the parameter. This is motivated by recognizing that spatial variability is

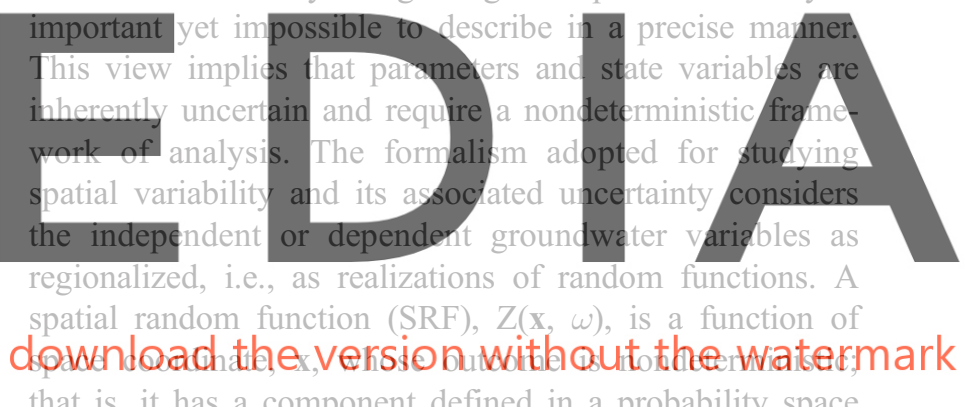

that is, it has a component defined in a probability space (this variation is represented by another "coordinate," $\omega$, which is usually suppressed to simplify notation). For a given point, $\mathbf{x}_{1}, Z\left(\mathbf{x}_{1}, \omega\right)$ is a random variable. For two given points, $\mathbf{x}_{1}$ and $\mathbf{x}_{2}, Z\left(\mathbf{x}_{1}, \omega\right)$ and $Z\left(\mathbf{x}_{2}, \omega\right)$ are two (generally) nonindependent random variables. Their dependence is usually characterized by the correlation between these two variables, which is a function of location and separation distance. On the other hand, fixing $\omega=\omega_{0}$ (i.e., providing an individual realization of the SRF) renders a single space function. The collection of all the space functions for the different $\omega$ values is called the ensemble. When spatial and ensemble averages coincide, the SRF is said to be ergodic. The topic of spatial random functions is a large one by itself and is needed for understanding much of what is written here. Fortunately, many comprehensive reviews are available, including those of Matheron [1965], Journel and Huijbregts [1978], Dagan [1989], Samper and Carrera [1990], Gelhar [1993], and Rubin [2003].

[9] In this paper we address saturated groundwater flow in both a deterministic and a stochastic framework. Under steady state the governing equation is obtained from mass balance and reads

$\nabla \cdot[\mathbf{K}(\mathbf{x}) \nabla \mathrm{h}(\mathbf{x})]=\mathrm{Q}(\mathbf{x})$ 
where Q is an external sink/source term. This equation is valid at a certain spatial scale, which is generally referred to as support volume.

[10] When the approach is stochastic, the variables that appear in (2) become random, so that we can formally write it as

$$
\nabla \cdot[\mathbf{K}(\mathbf{x}, \omega) \nabla \mathrm{h}(\mathbf{x}, \omega)]=\mathrm{Q}(\mathbf{x}) .
$$

Notice that considering the independent variable $\mathbf{K}$ as a SRF turns the dependent variable, $\mathrm{h}$, into another SRF, while Q can be either random (the notation would then be $\mathrm{Q}(\mathbf{x}, \omega)$ ) or deterministic. Equation (3) is then a stochastic partial differential equation.

\subsection{The Problem of Scales}

[11] The above definitions carry implicitly the validity of the flow equation at some local (support) scale. We will be seeking representative values of $\mathrm{K}$ at different scales. So some formalization is required regarding scales. Several attempts have been made at defining them. Dagan [1986, 1989] considered spatial variability at three different scales: pore, formation, and regional. This concept has been further elaborated by Gelhar [1993]. At the pore scale, physical variables are defined in terms of averages - ver a volume containing many inuum mechanics). In mode erogeneity at the pore scale local (or formation) mation thickness, and flow a three-dimensional nature
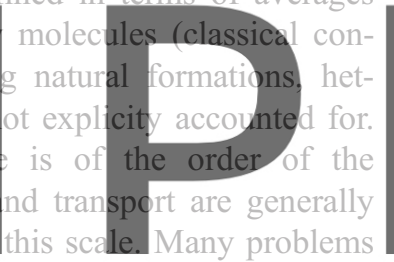

of interest in hydrogeology, such as pumping tests in the

larger than the aquifer thickness. Hydraulic parameters are averaged over depth, and flow is mainly two-dimensional. Local-scale variations can be smoothed out. This scale is of interest in applications such as management and longrange pollution.

[12] Variability of hydraulic conductivity at the local scale in sedimentary aquifers occurs over lengths (closely related to the concept of correlation distance) of the order of meters in the horizontal direction and about 1 order of magnitude smaller in the vertical direction [Gelhar, 1986]. On the other hand, characteristic lengths at the regional scale are of the order of hundreds to thousands of meters [Delhomme, 1979; Clifton and Neuman, 1982; Hoeksema and Kitanidis, 1985]. In fact, field evidence suggests that the correlation length of $\mathbf{K}(\mathbf{x})$ consistently increases with the domain size. This is in line with recent geological models based on process-imitating concepts and sequential stratigraphy [Teles et al., 2004, and references therein], and it suggests that stationarity of $\mathbf{K}(\mathbf{x})$ is not a real property of the medium but an artifact of the scale of observation. This prompted Neuman [1990, 1994] to model K as a process that is distributed over a continuous hierarchy of scales, forming a self-similar random variable with homogeneous increments.
[13] Normally, we are not interested in hydraulic heads or fluxes at individual volumes that coincide exactly with the support volume at which equation (3) was obtained. Thus we need to specify the equations that are valid at different (in our case larger) scales. At this stage we should ask ourselves some fundamental questions: (1) Is the formal structure of the equation maintained after a change in the scale of the problem? (2) If not, what is the proper representation of flow processes at a predefined scale? (3) If so, what are the different parameters values to be used at each scale? (4) Is there a way to bridge parameter values across different scales? Much of what follows in this paper is devoted to answering these questions. The traditional approach in hydrogeology, which might be questionable in some cases (as we will explore later), consists of applying to aquifers similar laws to those derived at the local scale. The issue is then to find the representative parameters to be used at that particular scale. The main question is, How representative are representative parameters? The answer is that ideally a representative parameter should yield an average behavior of the aquifer at a given scale. Depending on the averaging procedure, starting from Darcy's law we can obtain effective, equivalent, or interpreted parameters, defined as follows.

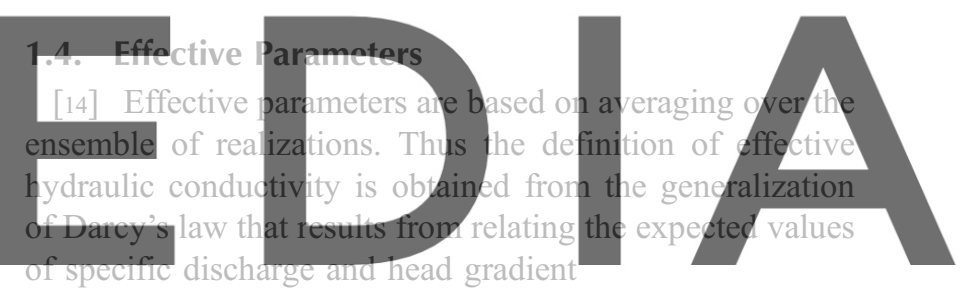

\section{download the version without the watermark}

where angle brackets indicate ensemble averaging in the probability space of hydraulic conductivity, that is, averaging all the possible head and specific discharge fields that could be obtained with the ensemble of hydraulic conductivity fields. If the value that relates both expecta-

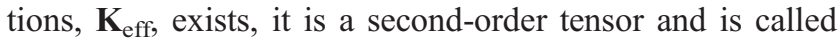
the effective hydraulic conductivity. The tensorial nature of $\mathbf{K}_{\text {eff }}$ may be a consequence of statistical anisotropy, boundary conditions, or domain geometry, as will be analyzed in sections 2.2 and 2.3 .

\subsection{Pseudoeffective or Apparent Parameters}

[15] The name effective is used only when the value appearing in (4) is constant throughout the domain. In this case the effective parameter can be considered as a characteristic property of the medium. The presence of boundaries or sink/sources would cause $\mathbf{K}_{\text {eff }}$ in (4) to be variable in space (actually, the most important variations would arise near the boundaries or the sources). Then even though (4) is still a proper definition, the resulting parameter is a function of space and cannot (in principle) be termed effective. In such cases some authors introduced the term pseudoeffective parameters [e.g., Sanchez-Vila, 1997]. Dagan [2001] coined the term "apparent conductivity" for this $\mathbf{K}_{\text {eff }}$ being a function of location, which has then been used in a 
number of publications [e.g., Riva et al., 2001; Tartakovsky et al., 2002; Guadagnini et al., 2003]. Still, we prefer to use the term "pseudoeffective" to emphasize that average quantities appearing in the definition of this parameter are computed in the probability space. The only difference with the concept of effective parameters is that a pseudoeffective value is not a characteristic of the medium but changes with location and is influenced by the presence of a boundary or by the nonstationarity of the medium (we will address this topic in sections 2.3, 2.6, and 2.8). We will use this terminology throughout the paper.

\subsection{Equivalent Parameters (or Block-Averaged Parameters)}

[16] A different approach to the problem relies on averaging in physical space. The resulting representative parameter is termed equivalent. Alternative terminologies that are often employed are block-averaged or volume-averaged parameters. Several authors refer to these as upscaled parameters, since these are usually representative of some average behavior observed over blocks larger than the support scale. Along these lines an equivalent hydraulic conductivity, $\mathrm{K}_{\mathrm{b}}$, can be defined by means of an averaged version of (1).

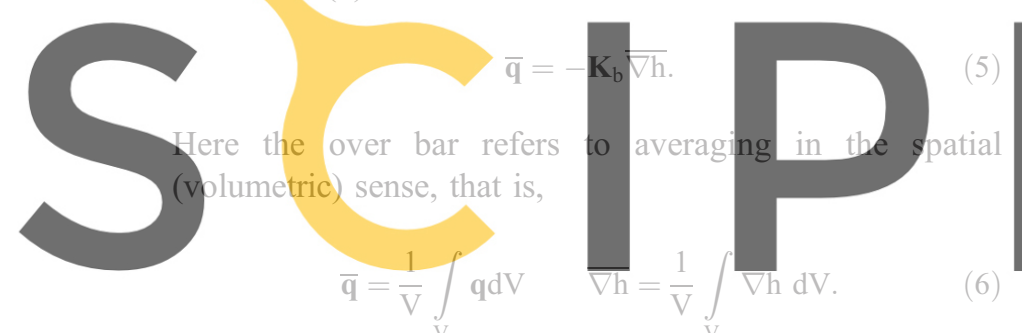

Register for free at https//www.scipedia.com to Even if $\mathrm{K}$ is isotropic at the local scale, upscaling may lead to a tensorial $\mathrm{K}_{\mathrm{b}}$. Notice that an expression equivalent to (5) would be

$$
\overline{\mathrm{K} \nabla \mathrm{h}}=\mathbf{K}_{\mathrm{b}} \overline{\nabla \mathrm{h}} .
$$

A discussion about conditions of existence and properties of such parameters will be presented in sections 3 and 4 .

[17] When the averaging volume is very large, with a representative size that is several times larger than the integral scale of the underlying (random) heterogeneous parameter, the averaging process comprises all scales of heterogeneity. In such a case, ergodicity would imply that equivalent and effective (or pseudoeffective) parameters be identical. A comprehensive discussion about conditions under which subsurface can be characterized by a unique integral scale within a predefined observation window is presented by Neuman and Di Federico [2003].

\subsection{Interpreted Parameters (or Estimated Parameters)}

[18] Interpreted parameters are again defined in physical space, as the ones obtained from the interpretation of field data, although this is often restricted to the interpretation of hydraulic tests. Thus they can also be termed estimated parameters. This causes the definition to be quite loose, as it involves interpretation issues. In general, tests are analyzed with some underlying simplifying hypotheses that are not completely met in reality. A clear example is the assumption of medium homogeneity, while reality is heterogeneous. When applying standard interpretation methods to a hydraulic test, one obtains a set of parameter values that are somehow "representative" of the global behavior of the aquifer at the scale of the test. The key point here is that the values obtained depend on the interpretation method and therefore do not have direct physical meaning. An example would be the estimation of transmissivity from a hydraulic test. Field hydrogeologists would interpret the test using a classical method (Theis or Cooper-Jacob) to obtain a value that they would later use in their models or calculations. Since interpreted parameters are the ones used in practice, the salient question here is whether interpreted parameters bear any relationship to effective, pseudoeffective, or equivalent parameters.

\subsection{Structure of the Paper}

[19] The objective of this paper is to review literature results regarding representative (i.e., effective, pseudoeffective, equivalent, and interpreted) hydraulic conductivities. Unfortunately, these concepts (including the existence and

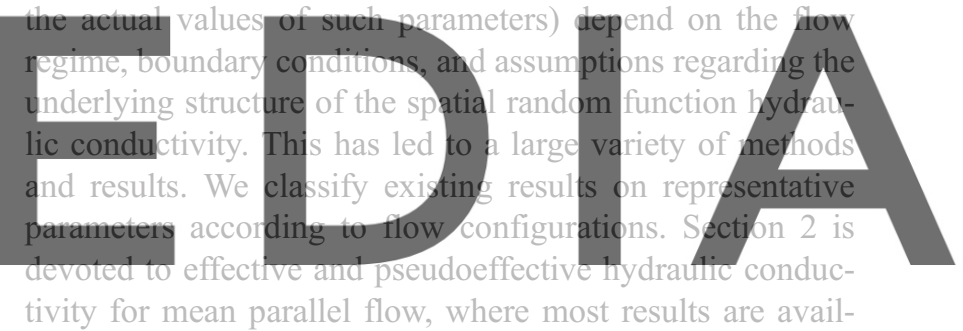

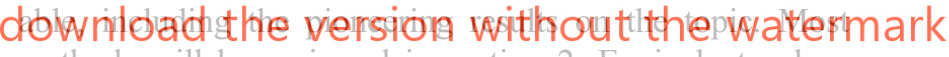 methods will be reviewed in section 2. Equivalent values} derived under the same flow conditions are presented in section 3. Section 4 is devoted to convergent flow, where, apart from effective, pseudoeffective, and equivalent parameters, interpreted values become relevant. A discussion on issues arising from scale effects is presented in section 4. Section 5 is devoted to other flow configurations. The paper ends with some discussion about relationships between classical and modern approaches and major results. A set of key conclusions and an appraisal of remaining challenges are then proposed.

\section{MEAN PARALLEL FLOW: EFFECTIVE AND PSEUDOEFFECTIVE PARAMETERS}

[20] This section is devoted to compiling the most important methodologies and relevant results available in the literature regarding effective and pseudoeffective hydraulic conductivity for mean parallel flow. Section 2 is structured over increasing levels of difficulty: It starts with isotropic, unbounded, and stationary media under steady state conditions and progresses to consider anisotropic media, bounded domains, and transient flow. The remainder of section 2 is devoted to different types of nonstationary media. Various reviews covering part of the topics 
addressed here are already available in the literature. The most recent ones are those of Dagan [2001], Rubin [2003], and Neuman and Di Federico [2003].

\subsection{Isotropic Stationary Media, Steady State Flow, and Unbounded Domains}

[21] As will become clear in the following, steady state parallel flow in stationary media is one of the particular situations where effective hydraulic conductivity exists.

\subsubsection{Bounds of the Principal Values of Effective Hydraulic Conductivity}

[22] The evaluation of $\mathbf{K}_{\mathrm{eff}}$ has been the subject of several studies since the 1960s. Matheron [1967] obtained the upper and lower bounds for $\mathrm{K}_{\mathrm{eff}}$ in any given direction by using energy dissipation considerations. He found that $\mathrm{K}_{\mathrm{eff}}$ was bounded by the arithmetic $\left(\mathrm{K}_{\mathrm{A}}\right)$ and the harmonic mean $\left(\mathrm{K}_{\mathrm{H}}\right)$ of the point values of conductivity. Mathematically, the principal values of the $\mathbf{K}_{\text {eff }}$ tensor are bounded by

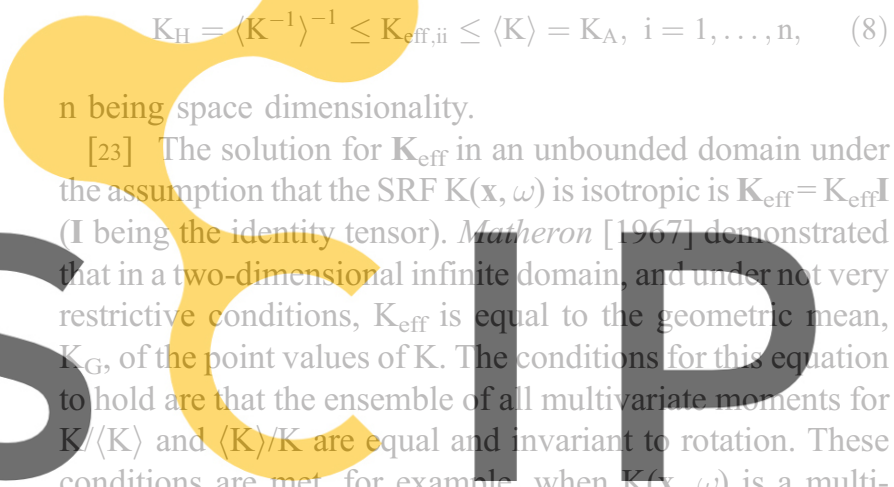

conditions are met, for example, when $\mathbf{K}(\mathbf{x}, \omega)$ is a multi

lognormal stationary random function with isotropic corre-

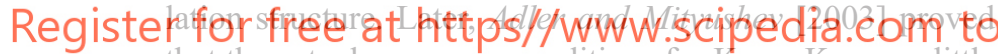
that the actual necessary conditions for $\mathrm{K}_{\mathrm{eff}}=\mathrm{K}_{\mathrm{G}}$ are a little less restrictive than those imposed by Matheron.

\subsubsection{Perturbative Methods}

2.1.2.1. Perturbations: General Ideas and Main Results

[24] Most of the results regarding effective parameters in heterogeneous media have been obtained using perturbation methods. This general methodology is widely applied in many other fields in science. It allows deriving analytical results or simplifying the numerical burden associated with Monte Carlo methods. The essence of small perturbations techniques is to expand the dependent variable in an asymptotic sequence and to derive different partial differential equations (PDEs) for each of the terms in the expansion. By solving them, we obtain the solution for the dependent variable (heads in the case of saturated groundwater flow) as a sequence. Closure and convergence analysis then become critical.

[25] The starting point consists of expanding the independent variable about its expected value, which is, in general, a function of $\mathbf{x}$. In hydrogeological problems, while some authors use as an independent variable $\mathrm{K}(\mathbf{x})$, most prefer to use $\mathrm{Y}(\mathbf{x})=\ln \mathrm{K}(\mathbf{x})$. In the latter case we can use Reynolds' decomposition to write

$$
\mathrm{Y}(\mathbf{x})=\langle\mathrm{Y}(\mathbf{x})\rangle+\mathrm{Y}^{\prime}(\mathbf{x}),
$$

where $\mathrm{Y}^{\prime}(\mathbf{x})$ is a zero-mean perturbation of the variable around its expected value, $\langle\mathrm{Y}(\mathbf{x})\rangle$. Exponentiating each side of equation (9) and noticing that $\mathrm{K}_{\mathrm{G}}(\mathbf{x})=\exp [\langle\mathrm{Y}(\mathbf{x})\rangle]$, an alternative expression for $\mathrm{K}$ reads: $\mathrm{K}(\mathbf{x})=\mathrm{K}_{\mathrm{G}}(\mathbf{x}) \exp \left[\mathrm{Y}^{\prime}(\mathbf{x})\right]$. In the special case of stationary media, $\mathrm{K}_{\mathrm{G}}$ is not a function of location.

[26] The next step consists in formally expanding $h(\mathbf{x})$ [Dagan, 1989]:

$$
\mathrm{h}(\mathbf{x})=\mathrm{h}^{(0)}(\mathbf{x})+\mathrm{h}^{(1)}(\mathbf{x})+\mathrm{h}^{(2)}(\mathbf{x})+\ldots
$$

Strictly speaking, an expansion of this kind, widely used in the stochastic literature, does not constitute an asymptotic sequence. This is so since random quantities defined on infinite probabilistic support, such as $\mathrm{h}^{(\mathrm{i})}(\mathbf{x})$, can take arbitrarily large values. The crucial points here are that the method relies on computing only statistical moments of the random variables involved, and moments are amenable to asymptotic expansions. Incorporating (9) and (10) into Darcy's law, and expanding $\exp \left(\mathrm{Y}^{\prime}(\mathbf{x})\right)$ into a Mac Laurin series, yields

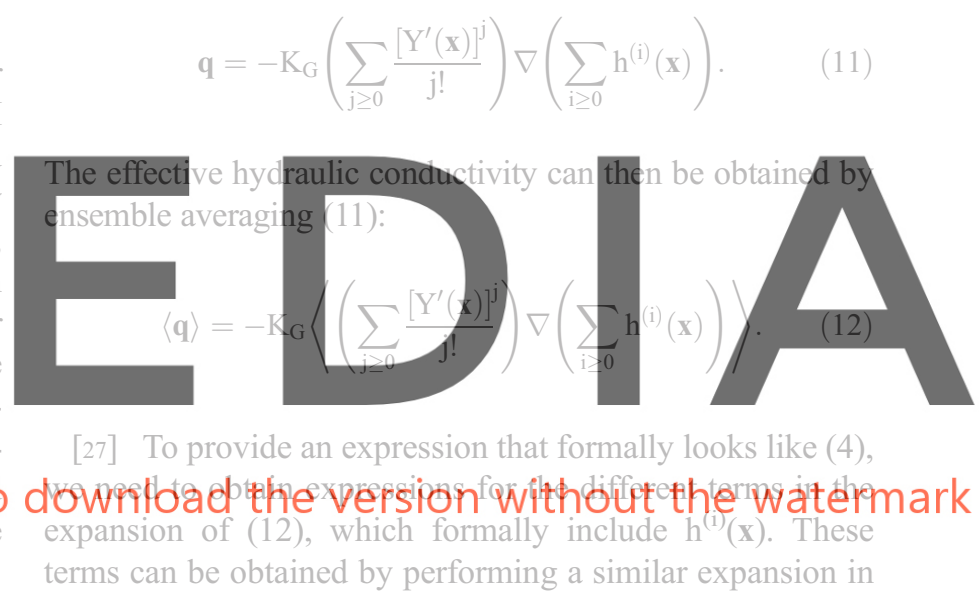

the groundwater flow equation (3), considering no external sources (even though they could be incorporated). Under conditions of stationarity in $\mathrm{Y}$ (i.e., $\nabla\langle\mathrm{Y}(\mathbf{x})\rangle=0$ ) we can write the steady state flow equation as the following set of sequential equations:

$$
\begin{gathered}
\nabla^{2} \mathrm{~h}^{(0)}(\mathbf{x})=0 \\
\nabla^{2} \mathrm{~h}^{(\mathrm{i})}(\mathbf{x})+\nabla \mathrm{Y}^{\prime}(\mathbf{x}) \nabla \mathrm{h}^{(\mathrm{i}-1)}(\mathbf{x})=0 \quad \mathrm{i} \geq 1 .
\end{gathered}
$$

[28] From (13), and noticing that the domain is infinite, $\mathrm{h}^{(0)}(\mathbf{x})$ is a deterministic function. Moreover, for mean uniform flow conditions, $\mathrm{h}^{(0)}(\mathbf{x}) \equiv\langle\mathrm{h}(\mathbf{x})\rangle$, arising from the fact that $\left\langle\mathrm{h}^{(\mathrm{j})}(\mathbf{x})\right\rangle=0$ for $\mathrm{j}>0$ [e.g., Dagan, 1989]. On the contrary, from (14) and as $\mathrm{Y}^{\prime}$ is an SRF, all the $\mathrm{h}^{(\mathrm{i})}(\mathbf{x}), \mathrm{i}=1$, $2, \ldots$ terms are the solution of the different stochastic PDEs and therefore are random as well. In (12), there is a double infinite series of ensemble averages of products of $\mathrm{Y}^{\mathrm{j}}$ and $\nabla h^{(i)}(\mathbf{x})$. Each term is assigned an order equal to $(\mathrm{i}+\mathrm{j})$. The terminology "small perturbations" in itself means that the terms of increasing order are of lesser significance. Using the "small perturbations" argument, we can truncate the 
expansion of (12). By retaining only terms up to second order in the expansion we arrive at

$$
\langle\mathbf{q}\rangle=-\mathrm{K}_{\mathrm{G}}\left[\left(1+\frac{\sigma_{\mathrm{Y}}^{2}}{2}\right) \nabla\langle\mathrm{h}(\mathbf{x})\rangle+\left\langle\mathrm{Y}^{\prime} \nabla \mathrm{h}^{(1)}(\mathbf{x})\right\rangle\right] .
$$

Here $\sigma_{\mathrm{Y}}^{2}$ is the variance of the natural logarithm, $\mathrm{Y}$, of hydraulic conductivity. An expression similar to (15) but formally incorporating all higher-order terms (before truncation) was already presented by Gelhar and Axness [1983]. When $\sigma_{\mathrm{Y}}^{2}$ is small, the small perturbation methodology applied to (12) converges to the exact solution, and truncation after a few terms, such as in (15), provides acceptable to very good results.

[29] The last step in the methodology is to write the last term on the right-hand side of (15) in terms of $\nabla\langle h(\mathbf{x})\rangle$. As we will show later in this section, this is generally not possible. Whenever it is possible, we actually arrive at an expression formally equal to (4), so that a low-order approximation for $\mathbf{K}_{\text {eff }}$ is readily obtained.

[30] The first applications of the methodology for the derivation of effective parameters were performed by Shvidler [1962] and subsequently by Matheron [1967]. These authors already concluded that
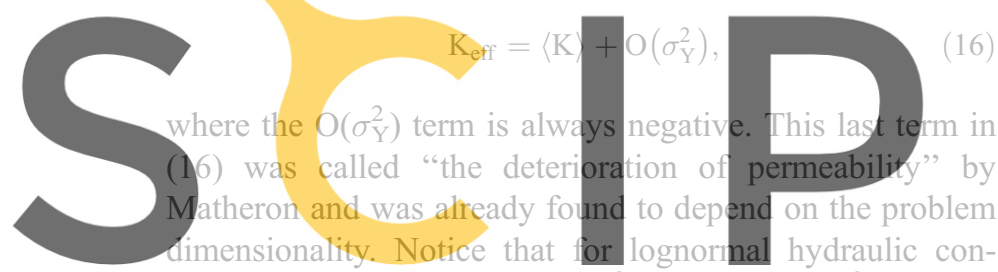

ductivity fields $\langle K\rangle=K_{G} \exp \left(\sigma_{Y}^{2} / 2\right) \simeq K_{G}\left(1+\sigma_{Y}^{2} / 2\right)$. Thus

Register there is a direct relation between (15) and (16) pointing out $\left.\nabla \mathrm{h}^{(1)}(\mathbf{x})\right\rangle$ term must be negative.

[31] The first compact expression for (15) for mean uniform flow conditions and stationary isotropic conductivity fields was presented by Gutjahr et al. [1978] as

$$
\left\langle\mathrm{Y}^{\prime} \nabla \mathrm{h}^{(1)}(\mathbf{x})\right\rangle=-\frac{\sigma_{\mathrm{Y}}^{2}}{\mathrm{n}} \nabla\langle\mathrm{h}(\mathbf{x})\rangle, \quad \nabla\langle\mathrm{h}(\mathbf{x})\rangle=\mathrm{constant},
$$

$\mathrm{n}$ being the number of space dimensions. Direct comparison of (15) and (4) allowed Gutjahr et al. to obtain the following expression for $\mathrm{K}_{\mathrm{eff}}$

$$
\mathrm{K}_{\mathrm{eff}}=\mathrm{K}_{\mathrm{G}}\left[1+\left(\frac{1}{2}-\frac{1}{\mathrm{n}}\right) \sigma_{\mathrm{Y}}^{2}\right] .
$$

[32] From (18), Gelhar and Axness [1983] conjectured an extrapolation valid for large $\sigma_{\mathrm{Y}}^{2}$ by suggesting that it is possible to consider (18) as the sum of the first two terms in the expansion of an exponential (this is known in hydrogeology as Matheron's conjecture [Matheron, 1967]), so that the equation becomes

$$
\mathrm{K}_{\mathrm{eff}}=\mathrm{K}_{\mathrm{G}} \exp \left[\left(\frac{1}{2}-\frac{1}{\mathrm{n}}\right) \mathrm{s}_{\mathrm{Y}}^{2}\right] .
$$

[33] This avoids the problem of having negative $\mathrm{K}_{\text {eff }}$ values in one dimension for large $\sigma_{\mathrm{Y}}^{2}$ values, as would be the case in (18).

[34] Assuming that the univariate distribution of $\mathrm{K}\left(\mathbf{x}_{0}\right)$ is lognormal, alternative equations to (19) are given as

$$
\begin{aligned}
& K_{\text {eff }}=K_{\mathrm{A}}{ }^{\frac{n-1}{n}} K_{H^{\frac{1}{n}}} \\
& K_{\text {eff }}=\left\langle K^{\frac{n-2}{n}}\right\rangle^{\frac{n}{n-2}} .
\end{aligned}
$$

Equation (20a) was already conjectured by Matheron [1967], even though it was not rigorously proved. Actually, Matheron arrives at a different analytical expression:

$$
\mathrm{K}_{\mathrm{eff}}=\frac{\mathrm{n}-1}{\mathrm{n}} \mathrm{K}_{\mathrm{A}}+\frac{1}{\mathrm{n}} \mathrm{K}_{\mathrm{H}}
$$

which is not correct. He found that (21) constituted a very low order approximation and conjectured (20a) as a potential exact solution to the problem. Equations (19) and (20a) are rigorously valid for $\mathrm{n}=1$, 2. Dagan [1993] derived, using small perturbations, the terms up to order $\sigma_{Y}^{4}$ for the expression of $\mathrm{K}_{\mathrm{eff}}$ and found these to be in agreement with the expansion of (19). Later, De Wit

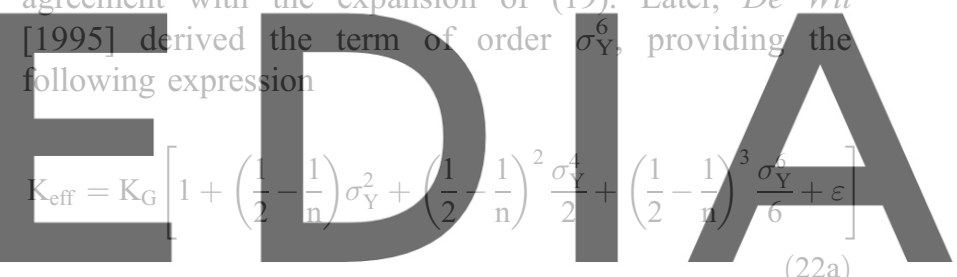

\section{download the version without the watermark}

The quantity $\varepsilon$ in (22b) vanishes for $n=1,2$. This is not the case for three-dimensional flows, where it is found numerically that $\varepsilon$ is approximately equal to $-0.0014 \sigma_{\mathrm{Y}}^{6}$ for a Gaussian log conductivity field. De Wit's solution is also valid for nonsymmetric log conductivity configurations, and then the value of $\beta$ is obtained as a ninedimensional integral. Figure 1 illustrates these results depicting the normalized effective conductivity for threedimensional mean uniform flows as obtained by means of the exponential conjecture (19) (solid curve) together with the sixth-order (in $\sigma_{\mathrm{Y}}$ ) approximation (22a) with $\beta=\sigma_{\mathrm{Y}}^{6} /\left(3 \mathrm{n}^{2}\right)$ (dash-dotted curve) and $\varepsilon=-0.000467 \sigma_{\mathrm{Y}}^{6}$ or $\varepsilon=-0.0014 \sigma_{\mathrm{Y}}^{6}$ for $\log$ conductivity field characterized by an exponential (dashed curve) or Gaussian (dotted curve) covariance function, respectively. Similar methodology and results for the Gaussian case were presented at about the same time by Abramovich and Indelman [1995].

\subsubsection{Small Perturbations Combined With Green's Functions}

[35] Several methods can be used to derive (17). Because of their applicability to more complex problems we present the main steps of some of these methodologies when 


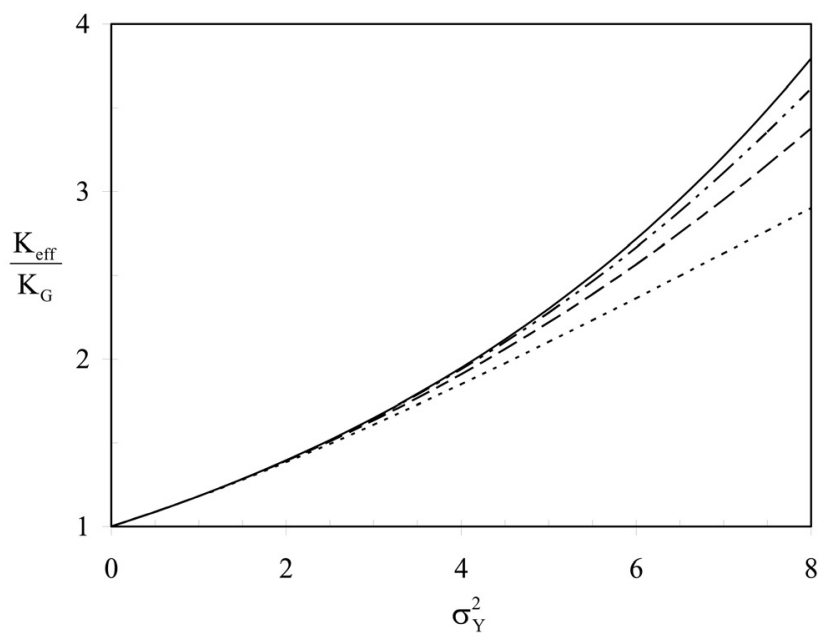

Figure 1. Normalized effective conductivity for threedimensional mean uniform flows as obtained by means of the exponential conjecture (19) (solid curve). For comparison, the dash-dotted curve is the sixth-order (in $\sigma_{\mathrm{Y}}$ ) approximation (22a) with $\beta=\sigma_{\mathrm{Y}}^{6} /\left(3 \mathrm{n}^{2}\right)$, and the dashed and dotted curves are (22a) with $\varepsilon=-0.000467 \sigma_{\mathrm{Y}}^{6}$ or $\varepsilon=$ $-0.0014 \sigma_{\mathrm{Y}}^{6}$, representing a log conductivity field characterized by an exponential or Gaussian covariance function, respectively. Reprinted with permission from De Wit [1995].

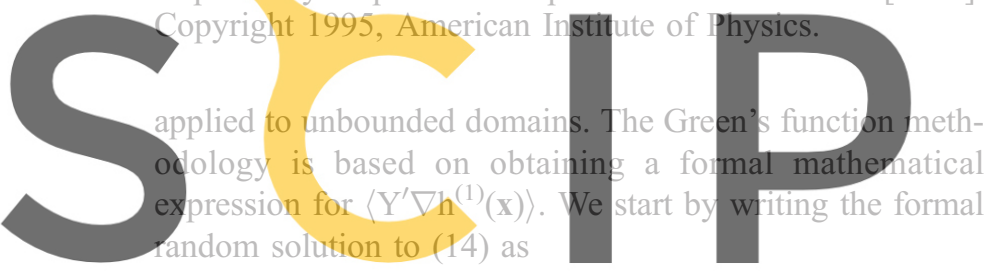

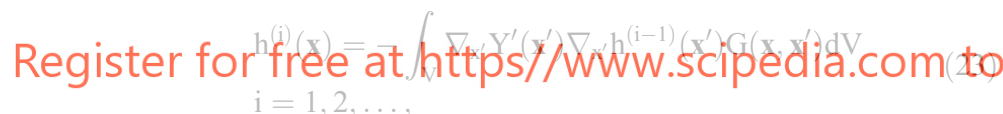

where $V$ is the domain and $G\left(\mathbf{x}, \mathbf{x}^{\prime}\right)$ is the deterministic Green's function for the Laplace equation in V, i.e., the solution to

$$
\nabla^{2} \mathrm{G}\left(\mathbf{x}, \mathbf{x}^{\prime}\right)=-\delta\left(\left|\mathbf{x}-\mathbf{x}^{\prime}\right|\right)
$$

in $\mathrm{V}$, subject to homogeneous boundary conditions. Some boundary integrals also appear in the general solution in a bounded domain. From (24), and taking advantage of the fact that $\nabla \mathrm{h}^{(0)}(\mathbf{x})=\nabla\langle\mathrm{h}(\mathbf{x})\rangle=$ constant, we can write

$$
\left\langle\mathrm{Y}^{\prime} \nabla \mathrm{h}^{(1)}(\mathbf{x})\right\rangle=\left[-\int_{\mathrm{V}}\left\langle\mathrm{Y}^{\prime}(\mathbf{x}) \nabla_{\mathbf{x}^{\prime}} \mathrm{Y}^{\prime}\left(\mathbf{x}^{\prime}\right)\right\rangle \nabla \mathrm{G}\left(\mathbf{x}, \mathbf{x}^{\prime}\right) \mathrm{dV}\right] \nabla\langle\mathrm{h}\rangle .
$$

Now the integral appearing in (25) is a deterministic quantity and can be evaluated analytically or numerically. Evaluating (25) in unbounded domains would lead to (17).

[36] A very similar approach, also based on Green's function, was taken initially by Shvidler [1962] and Matheron [1967] in the study of effective parameters under different flow configurations and was applied to other problems by Dagan [1982, 1987]. The methodology was explained in detail by Dagan [1989].

\subsubsection{Small Perturbations Combined With Covariance Spectra}

[37] An alternative to the use of Green's functions is based on the spectral representation theorem. Under the condition that $\mathrm{K}(\mathbf{x}, \omega)$ is a stationary random function, both $\mathrm{Y}^{\prime}(\mathbf{x}, \omega)$ and $h^{(1)}(\mathbf{x}, \omega)$ can be represented through a Fourier-Stieltjes integral [Gelhar, 1986]. Then, the first stochastic PDE in (14) becomes a linear equation with constant coefficients in the spectral space. It is then possible to write the spectral density of hydraulic heads from the spectral density of $\log$ conductivities. This approach has been successfully used in works by Bakr et al. [1978], Mizell et al. [1982], and Gelhar and Axness [1983] among many others. The methodology was explained in detail by Gelhar [1993].

[38] The spectral representation theorem states that any stationary SRF (in general, a stationary random process) can be written in terms of a stochastic process, Z, with orthogonal increments, as follows:

$$
\mathrm{Y}^{\prime}(\mathbf{x})=\int_{-\infty}^{+\infty} \exp (\mathrm{i} \mathbf{k} \cdot \mathbf{x}) \mathrm{d} Z_{Y}(\mathbf{k})
$$

Here $\mathbf{k}=\left(\mathrm{k}_{1}, \mathrm{k}_{2}, \mathrm{k}_{3}\right)$ is the wave vector in spectral space. By using the same type of representation for $h^{(1)}(\mathbf{x})$ and from EDIA

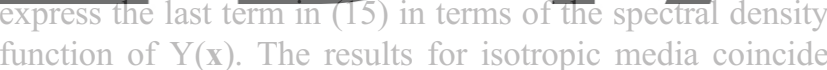

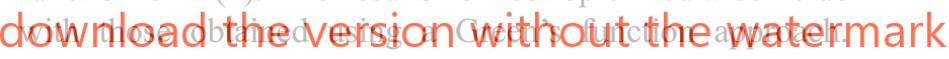
Applications of the methodology to bounded domains lead to a truncated, and thus approximated, spectral representation of the random processes involved.

\subsubsection{Residual Flux Theory Approach}

[39] Neuman and coworkers [Neuman and Orr, 1993; Neuman et al., 1996; Tartakovsky and Neuman, 1998a, 1998b; Guadagnini and Neuman, 1999a, 1999b; Riva et al., 2001; Tartakovsky et al., 2002; Guadagnini et al., 2003] analyzed effective and pseudoeffective hydraulic conductivity by means of moment equations of transient and steady state groundwater flow. Under steady state the predictor of the flux, $\langle\mathbf{q}\rangle$, is written as

$$
\langle\mathbf{q}\rangle=-\langle\mathrm{K}\rangle \nabla\langle\mathrm{h}\rangle+\mathbf{r} ; \quad \mathbf{r}=-\left\langle\mathrm{K}^{\prime} \nabla \mathrm{h}^{\prime}\right\rangle,
$$

where it can be easily recognized that $\langle\mathbf{q}\rangle$ is expressed in terms of a Darcian component, which is governed by the hydraulic conductivity predictor, $\langle\mathrm{K}\rangle$, and a (generally) nonDarcian one, which is termed residual flux, $\mathbf{r}$. It has been shown by Neuman and Orr [1993] and Neuman et al. [1996] that for a bounded domain, $\Omega$, with impermeable Neumann boundaries, the residual flux is given exactly by the compact explicit expression

$$
\mathbf{r}(\mathbf{x})=\int_{\Omega} \mathbf{a}(\mathbf{y}, \mathbf{x}) \nabla_{\mathrm{y}} \mathrm{h}_{\mathrm{c}}(\mathbf{y}) \mathrm{d} \mathbf{y}
$$


Here $h_{c}$ is the solution of the following deterministic flow problem

$$
\nabla \cdot\left[\langle\mathrm{K}(\mathbf{x})\rangle \nabla \mathrm{h}_{\mathrm{c}}(\mathbf{x})\right]+\langle\mathrm{f}(\mathbf{x})\rangle=0,
$$

where $\langle f(\mathbf{x})\rangle$ is the mean source term and the boundary conditions are provided in terms of (ensemble) mean quantities. The kernel

$$
\mathbf{a}(\mathbf{y}, \mathbf{x})=\left\langle\mathrm{K}^{\prime}(\mathbf{x}) \mathrm{K}^{\prime}(\mathbf{y}) \nabla_{\mathrm{x}} \nabla_{\mathrm{y}}^{\mathrm{T}} \mathrm{G}_{\mathrm{r}}(\mathbf{y}, \mathbf{x})\right\rangle
$$

is a second-rank positive semidefinite symmetric tensor, $\mathrm{G}_{\mathrm{r}}(\mathbf{y}, \mathbf{x})$ being the random Green's function associated with the groundwater flow problem, i.e., the solution of the (random) groundwater flow equation for the case where the source function is replaced by the Dirac delta function $\delta(\mathrm{x}-\mathrm{y})$, subject to homogeneous boundary conditions [Greenberg, 1971]. In the general case of prescribed nonzero (random) flux along Neumann boundaries, (29) has to be supplemented with a series of boundary integrals. As an alternative the residual flux can be expressed by means of an implícit expression:

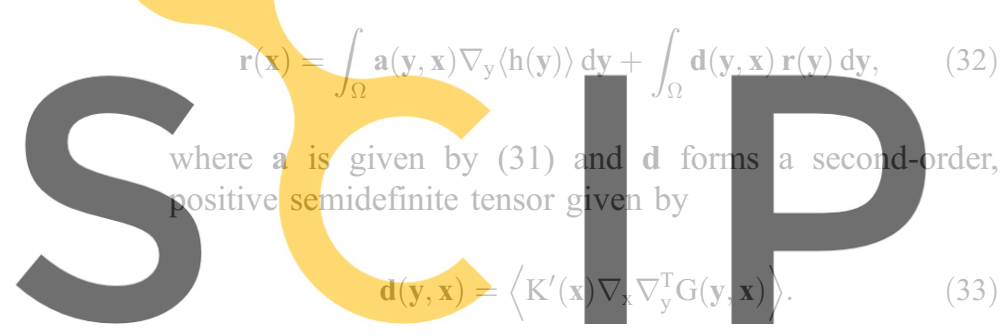

It is precisely the integrodifferential nature of the residual

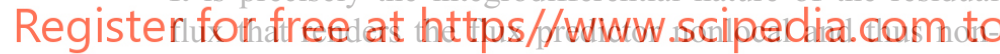
Darcian, so that a (pseudo)effective conductivity does not exist with the exception of a few particular cases. This is a very important point to emphasize, since the methodologies directly based on perturbations of random quantities do not allow recognizing general conditions of existence of effective conductivities. A discussion about generally nonuniform flow configurations will be presented in sections 4 and 5. In the special case of flow in an infinite domain with (statistically) homogeneous hydraulic conductivity under a uniform mean hydraulic gradient $\nabla \mathrm{h}_{\mathrm{c}}=\nabla\langle\mathrm{h}\rangle=$ const, the second integral in (32) must vanish, and therefore the residual flux can be localized exactly, i.e., $\mathbf{r}(\mathbf{x})=-\mathrm{K}_{\mathrm{C}}(\mathbf{x}) \nabla\langle\mathrm{h}(\mathbf{x})\rangle$.

[40] Owing to symmetry considerations, applicable only to infinite domains, $\mathrm{K}_{\mathrm{C}}(\mathbf{x})$ is constant throughout the domain, and an effective conductivity value can be defined as

$$
\mathbf{K}_{\text {eff }}=\langle\mathrm{K}\rangle \mathbf{I}-\mathbf{K}_{\mathrm{C}}, \quad \mathbf{K}_{\mathrm{C}}=\mathbf{K}_{\mathrm{C}}(\mathbf{x})=\int_{\Omega} \mathbf{a}(\mathbf{y}, \mathbf{x}) \mathrm{d} \mathbf{y} .
$$

The salient feature of this expression is that the evaluation of the integral term in (34) requires a closure model. Closure can be obtained by a large eddy diffusivity approximation [e.g., Kraichnan, 1987], leading to

$$
\mathbf{a}(\mathbf{y}, \mathbf{x}) \approx\left\langle\mathrm{K}^{\prime}(\mathbf{x}) \mathrm{K}^{\prime}(\mathbf{y})\right\rangle \nabla_{\mathrm{x}} \nabla_{\mathrm{y}}^{\mathrm{T}} \mathrm{G}(\mathbf{y}, \mathbf{x}),
$$

where $\mathrm{G}$ is a deterministic Green's function defined as the solution of the deterministic groundwater flow equation, with $\mathrm{K}=\langle\mathrm{K}\rangle$. Alternatively, one can use perturbation expansion techniques. By doing so, the equations recovered for infinite domain provide the same solutions as the methodologies presented so far. We emphasize that a key theoretical advantage of the residual flux-based approach is that the variables expanded are deterministic moments rather than random quantities. Expansion of moments is performed in series of a parameter representing a measure of the standard deviation of log-hydraulic conductivity, the smallness of which can be assessed. Key advantages of this methodology, as opposed to the others, are that (1) it does not require restrictions about the probability density function (pdf) of $\mathrm{K}$ and (2) it can incorporate conditioning on different types of information. An important annlication is the possibility of addressing bounded domains, as we will see in section 2.3. 2.1.2.5. Other Perturbative Techniques

[41] Another method also based on perturbations is stochastic diagrammatic analysis. The basic idea is to perform a perturbation expansion similar to the one defined by (9) and (10). The difference lies in the way the resulting equations are treated. The resulting terms involving the integral of $n$-point correlations are presented in a concise

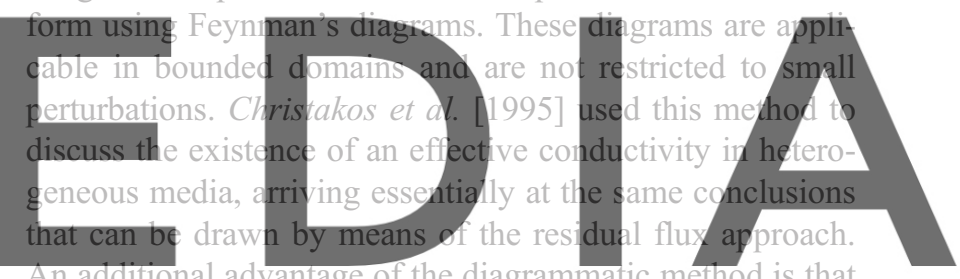
An additional advantage of the diagrammatic method is that it allows obtaining results for partial summations at any downdardn the vernsion fwith outptomeliwatermark insight to the solution in a number of cases. These partial summations cannot provide the full solution to any given order, and therefore the method is limited when applied to obtaining higher-order results in closed form.

[42] Stepanyants and Teodorovich [2003] used a different perturbative approach based on the decomposition of seepage velocity into a potential plus a solenoidal function. Then they expanded the product $\mathrm{K}^{-1}(\mathbf{x}) \mathbf{q}(\mathbf{x})$ in terms of perturbations. Using a diagrammatic method, they can compute the low-order terms of the effective conductivity, providing the following result:

$$
\mathrm{K}_{\mathrm{eff}}=\mathrm{K}_{\mathrm{G}} \exp \left(-\sigma_{\mathrm{Y}}^{2} / 2\right)\left[1-\frac{\mathrm{n}-1}{\mathrm{n}} \sigma_{\mathrm{Y}}^{2}+\frac{1}{2}\left(\frac{\mathrm{n}-1}{\mathrm{n}}\right)^{2} \sigma_{\mathrm{Y}}^{4}\right]^{-1} .
$$

This result cannot be directly applied to large $\sigma_{\mathrm{Y}}^{2}$ values, since these would provide a decreasing $\mathrm{K}_{\text {eff }}$ value for increasing degree of heterogeneity in three dimensions. In addition to this it does not provide the exact solution $\mathrm{K}_{\mathrm{eff}}=$ $\mathrm{K}_{\mathrm{G}}$ in two dimensions. On the other hand, when Matheron's conjecture is applied to (36), the final results are in agreement with (19).

[43] Another perturbative technique was initially used by Noetinger [1994]. His approach consisted of expanding in a 
geometrical series the inverse of a linear integral operator that involves a Green's series. Noetinger then writes the effective conductivity as the sum of an infinite number of terms:

$$
\mathrm{K}_{\mathrm{eff}}=\sum_{\mathrm{N}=0}^{\infty} \mathrm{K}_{\mathrm{N}}
$$

where each $\mathrm{K}_{\mathrm{N}}$ term includes high-order correlation functions such as $\left\langle\exp \left(\mathrm{Y}^{\prime}(\mathbf{r}) \mathrm{Y}^{\prime}\left(\mathbf{r}_{1}\right) \ldots \mathrm{Y}^{\prime}\left(\mathbf{r}_{\mathrm{N}}\right)\right\rangle\right.$. For lognormal conductivity media, Noetinger [1994] summed up all the terms that do not include correlations between different points. This is equivalent to finding the exact expression for $\mathrm{K}_{\text {eff }}$ for vanishing correlation length. The final expression corresponds exactly to (19), thus revealing that (19) is an exact result for negligible correlation length. Unfortunately, this methodology provides no additional insight with respect to other methodologies when applied to correlated conductivity fields.

\subsubsection{Nonperturbative Methods}

2.1.3.1. Self-Consistent Approach

[44] The self-consistent approach, also termed embedding matrix approach [Dagan, 1981], is based on the following idea: We start with a homogenized medium with a hydraulic

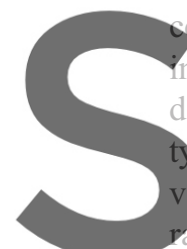

onductivity value equal to

clusion is incorporated int

dimension of the inclusion de

ypically an $n$-dimensional sp

value assigned to the inclusion, $K_{\text {in }}$

andom from a predefined pdf of $\mathrm{K}, \mathrm{p}(\mathrm{K})$. For each

particular value of $\mathrm{K}_{\text {inclusion, }}$ there is a different head distribution solution. The $\mathrm{K}_{\text {eff }}$ value is defined as that of a

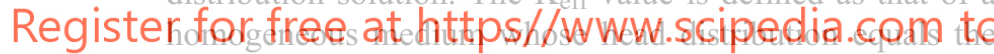

average of the inclusion head distributions. It is clear from the definition that the solution must always be obtained through an iterative process. In an isotropic medium, Dagan [1981] obtained the following solution for $\mathrm{K}_{\mathrm{eff}}$ :

$$
\mathrm{K}_{\mathrm{eff}}=\frac{1}{\mathrm{n}}\left[\int_{0}^{\infty} \frac{\mathrm{p}(\mathrm{K})}{\mathrm{K}+(\mathrm{n}-1) \mathrm{K}_{\mathrm{eff}}} \mathrm{dK}\right]^{-1} .
$$

This methodology is not limited to small variances of the $\log$ conductivity field. Moreover, if $\mathrm{p}(\mathrm{k})$ is assumed to be lognormal, it provides the exact solution both for the one$\left(\mathrm{K}_{\text {eff }}=\mathrm{K}_{\mathrm{H}}\right)$ and the two-dimensional $\left(\mathrm{K}_{\mathrm{eff}}=\mathrm{K}_{\mathrm{G}}\right)$ cases [Dagan, 1993]. However, equation (38) leads to an underestimation of $\mathrm{K}_{\mathrm{eff}}$ in three dimensions. The selfconsistent approach has been widely used to derive effective parameters in anisotropic media and bimodal conductivity fields, as will be presented in sections 2.2 and 2.7.

\subsubsection{Method of Moments}

[45] The method of moments [Kitanidis, 1990] takes advantage of the formalism of periodic media. Kitanidis assumed that the conductivity field is periodic in the threedimensional space and that the period in all three directions is known. Ergodicity can then be invoked by setting the periodic distance to infinity. The equivalence of ensemble parameters to those obtained by large periodization volume averaging has been demonstrated rigorously by Owhadi [2003]. The method consists of averaging out in real space (not in the ensemble) the effects of gradually varying flow (steady state being the limit for infinite time). Under these conditions, Kitanidis [1990] obtains that the effective conductivity is given by a volume integral over one single period. The volume integral involves functions that satisfy a Poisson-type PDE with periodic boundary conditions. By considering a volume which tends to infinity, he recovers an expression for $\mathrm{K}_{\mathrm{eff}}$ which coincides with (18). This method involves a rigorous mathematical formalism, and though it has not been widely used in the hydrogeological literature, it is commonly applied in other branches of physics.

\subsubsection{Variational Method}

[46] The variational method consists of writing the actual expression for effective conductivity in Fourier space in order to eliminate the nonlocalities that arise from the solution to the flow equation using Green's functions. Auxiliary correlation functions are obtained through variational principles in an optimization process. Using this method, Hristopulos and Christakos [1997] provided an expression for effective hydraulic conductivity in an $n$ dimensional isotropic unbounded domain,

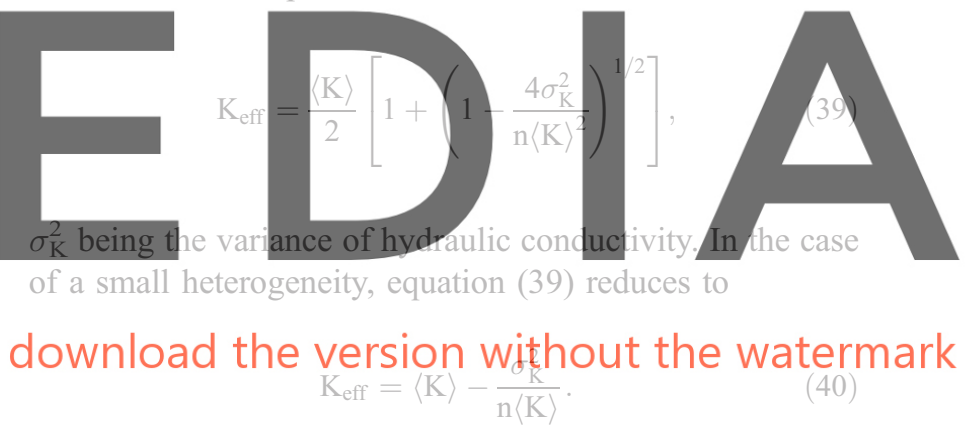

According to Bulgadaev [2003], equation (40) was already obtained by Landau and Lifshitz [1960] in the field of electrodynamics. The solutions provided in (40) are subject to serious restrictions. For instance, in the case of lognormal hydraulic conductivity these reduce to

$$
\begin{gathered}
\mathrm{K}_{\text {eff }}=\frac{\mathrm{K}_{\mathrm{G}}}{2} \exp \left(\sigma_{\mathrm{Y}}^{2} / 2\right)\left\{1+\sqrt{1-\frac{4}{\mathrm{n}}\left[\exp \left(\sigma_{\mathrm{Y}}^{2}\right)-1\right]}\right\} \\
\mathrm{K}_{\mathrm{eff}}=\mathrm{K}_{\mathrm{G}} \exp \left(\sigma_{\mathrm{Y}}^{2} / 2\right)\left\{1-\frac{1}{\mathrm{n}}\left[\exp \left(\sigma_{\mathrm{Y}}^{2}\right)-1\right]\right\} .
\end{gathered}
$$

Then, for two-dimensional flow, values of $\sigma_{\mathrm{Y}}^{2}$ larger than 0.4 would lead to imaginary values for $\mathrm{K}_{\text {eff }}$ in (41a), while $\sigma_{\mathrm{Y}}^{2}>1.1$ leads to negative values in (41b). Similar restrictions apply in three dimensions. All these results contradict the claim of Hristopulos and Christakos [1997] that the solution is not restricted to moderate heterogeneity.

\subsubsection{Renormalization Methods}

[47] Noetinger [1994, 2000] used a renormalization group method to develop the $\mathrm{K}_{\text {eff }}$ value for a lognormal 
hydraulic conductivity field. The approach consists of defining a cutoff value in Fourier space, $\Lambda$, and then finding an analytical relationship between $\mathrm{K}_{\text {eff }}(\Lambda+\mathrm{d} \Lambda)$ and $\mathrm{K}_{\text {eff }}(\Lambda)$. By considering the limit of $\Lambda$ which tends to zero, Noetinger found out that (19) is an exact result, thus criticizing the results of De Wit [1995] obtained by a perturbations approach. Since De Wit's correction is very small for the usual range of $\sigma_{\mathrm{Y}}^{2}$ values found in practical applications, this discrepancy between the two methods is very difficult to assess and not completely significant.

\subsubsection{Other Methods}

[48] Madden [1976] modeled two-dimensional heterogeneous media as a random network and concluded that the geometric mean can be used to predict conductivity of heterogeneous mixtures of materials. His result is in line with later findings that the geometric mean of locally random hydraulic conductivities can be used, under certain flow conditions, as representative of the overall conductivity of the medium.

[49] An approach in principle similar to that of Noetinger [1994] was presented by Teodorovich [1997, 2000]. Again, the idea is to expand the inverse of an operator. While the approach of Noetinger [1994] was in terms of perturbations, Teodorovich [1997, 2000] wrote it in terms of a Feynman

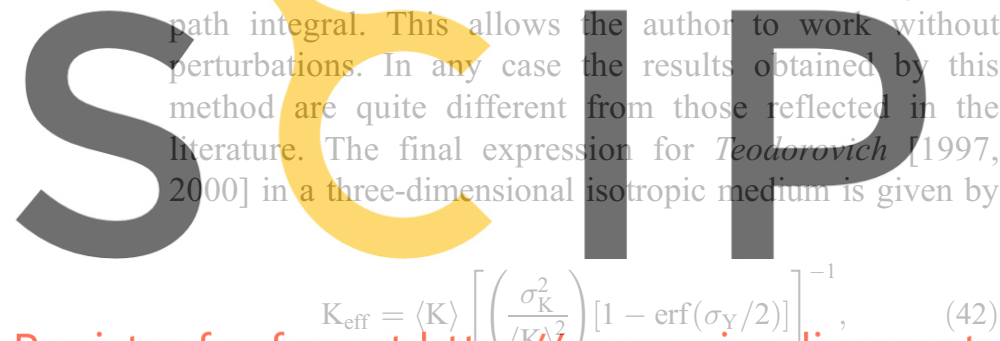

Register for free at httpsy)/www.scipedia.com to

where $\operatorname{erf}($ ) stands for error function. This solution is not in agreement with solutions presented in section 2.1 (and validated numerically), thus questioning the applicability of the method to the problem of finding effective conductivities.

\subsection{Anisotropic Media}

[50] The limiting case for an anisotropic medium is that of a perfectly stratified aquifer. When a head gradient is imposed parallel to stratification, the total flow equals that of a homogeneous medium with hydraulic conductivity $\mathrm{K}_{\mathrm{A}}$ (arithmetic mean of point $\mathrm{K}$ values). Contrariwise, if flow is perpendicular to stratification, the homogenous counterpart for hydraulic conductivity would be $\mathrm{K}_{\mathrm{H}}$. These results can be traced back at least to Terzaghi and Peck [1948]. Invoking ergodic arguments, we can state that the effective hydraulic conductivities for flow parallel or perpendicular to stratification are $\langle\mathrm{K}\rangle$ and $\left\langle\mathrm{K}^{-1}\right\rangle^{-1}$, respectively.

[51] We return now to fields where heterogeneity is not deterministic. Let us consider a heterogeneous domain where $\mathrm{K}(\mathbf{x}, \omega)$ is taken as locally isotropic but statistically anisotropic, meaning that a covariance structure exists and is directionally dependent. Let $\lambda_{1}, \lambda_{2}$, and $\lambda_{3}$ be the correlation scales in the principal directions of correlation.
If the global coordinate system $\left(\mathrm{x}_{1}, \mathrm{x}_{2}, \mathrm{x}_{3}\right)$ is taken to coincide with these principal directions, the effective conductivity expressed in these coordinates will exist and will be a diagonal tensor.

[52] To our knowledge, Gelhar and Axness [1983] were the first to address the problem of effective conductivity in a two-dimensional anisotropic infinite domain under mean uniform flow conditions (actually they did it as the limiting case $\lambda_{3} \rightarrow \infty$ in a three-dimensional problem). They found that $\mathbf{K}_{\text {eff }}$ is a diagonal tensor whose principal components are given by

$$
\mathrm{K}_{\mathrm{eff}, \mathrm{i}}=\mathrm{K}_{\mathrm{G}}\left[1+\sigma_{\mathrm{Y}}^{2}\left(\frac{1}{2}-\frac{\lambda_{\mathrm{j}}}{\lambda_{1}+\lambda_{2}}\right)\right], \quad \mathrm{i}, \mathrm{j}=1,2 \text { and } \mathrm{i} \neq \mathrm{j} .
$$

It is more convenient to write the solution to the effective conductivity for the two-dimensional case in terms of the anisotropy ratio of the correlation structure. If we define the anisotropy ratio e $=\lambda_{2} / \lambda_{1}(\leq 1)$ and we further consider (43) as the first terms of an exponential expansion, we can write
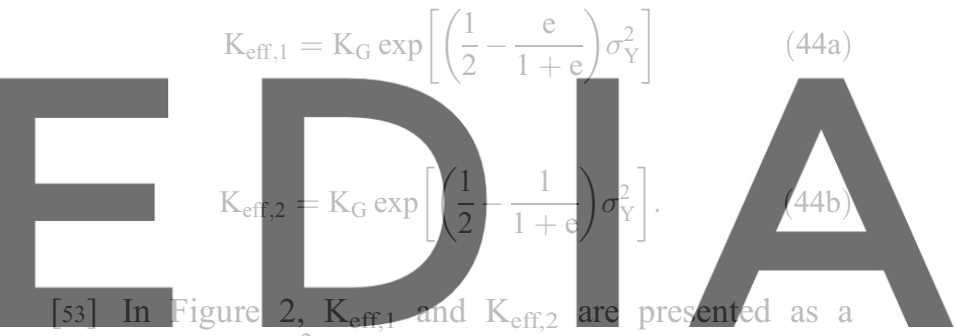

function of e and $\sigma_{\mathrm{Y}}^{2}$ based on (44). The asymptotic limits are wel, known. For e $=1$.we get $\mathrm{K}_{\text {are }}=\mathrm{K}_{\mathrm{fr}}=\mathrm{K}_{\mathrm{G}}$ thus downhod the version without the watermark

$\mathrm{e} \rightarrow 0$, which is equivalent to a layered system and allows recovering $\mathrm{K}_{\mathrm{eff}, \mathrm{1}}=\langle\mathrm{K}\rangle$ and $\mathrm{K}_{\mathrm{efr}, 2}=\left\langle\mathrm{K}^{-1}\right\rangle^{-1}$. Other interesting results that can be extracted from (44a) are that for any given value of the anisotropic ratio, e, we obtain

$$
\sqrt{\mathrm{K}_{\mathrm{eff}, 1} \cdot \mathrm{K}_{\mathrm{eff}, 2}}=\mathrm{K}_{\mathrm{G}}
$$

$$
\frac{\mathrm{K}_{\mathrm{eff}, 1}}{\mathrm{~K}_{\mathrm{eff}, 2}}=\exp \left(\frac{1-\mathrm{e}}{\mathrm{e}+1} \sigma_{\mathrm{Y}}^{2}\right)
$$

[54] The last expression shows that the nonlinear relationship between the anisotropy ratios of the effective hydraulic conductivity and that of the $\log$ conductivity correlation scales [Sanchez-Vila and Carrera, 1997]. From (45), and if one has information about e (coming from geostatistical analysis of data or geological analogues) and $\sigma_{\mathrm{Y}}^{2}$ and $\mathrm{K}_{\mathrm{G}}$ (coming from laboratory- or field-based data), it would be possible (in principle) to evaluate the individual directional effective parameters and assess the principle directions of the effective conductivity tensor.

[55] Since Gelhar and Axness [1983] several authors have considered the case of anisotropic correlation structures in three dimensions. The closed-form results were 


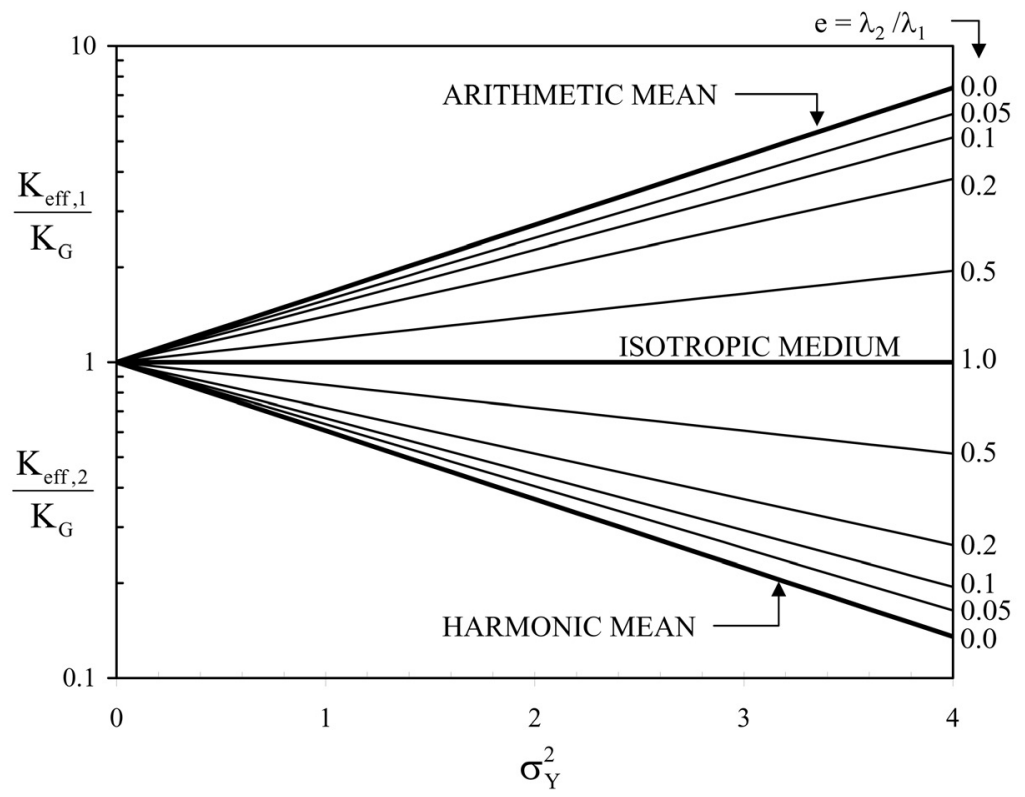

Figure 2. Dependence of horizontal and vertical effective conductivities, $\mathrm{K}_{\mathrm{eff}, 1}(44 \mathrm{a})$ and $\mathrm{K}_{\mathrm{eff}, 2}(44 \mathrm{~b})$, respectively, on the anisotropy ratio, e, and log conductivity variance, $\sigma_{\mathrm{Y}}^{2}$.

presented by Dagan [1989] for the case of an axisymmetric covariance function, with $\lambda_{1}=\lambda_{2}$ and $\mathrm{e}=\lambda_{3} / \lambda_{1}$. The corresponding result, relying on a perturbation expansion truncated to second order in $\sigma_{\mathrm{Y}}$, is

$$
\begin{aligned}
& \mathrm{K}_{\mathrm{eff}, 1}=\mathrm{K}_{\mathrm{eff}, 2}=\mathrm{K}_{\mathrm{G}}\left(1+\frac{\mathrm{c}}{2} \sigma_{\mathrm{Y}}^{2}\right) \\
& \mathrm{K}_{\mathrm{eff}, 3}=\mathrm{K}_{\mathrm{G}}\left[1+\left(\frac{1}{2}-\chi\right) \sigma_{\mathrm{Y}}^{2}\right],
\end{aligned}
$$

with

$$
\chi=\frac{1}{1-\mathrm{e}^{2}}\left(1-\frac{\mathrm{e}}{\sqrt{1-\mathrm{e}^{2}}} \tan ^{-1} \sqrt{\frac{1}{\mathrm{e}^{2}}-1}\right) \quad \text { for } \mathrm{e}<1 .
$$

Expression (47) was provided by Dagan [1989] with a different notation. Using again the argument that the terms in (46) are the first terms in an exponential expansion, we arrive at the following formulae:

$$
\begin{aligned}
& \mathrm{K}_{\mathrm{eff}, 1}=\mathrm{K}_{\mathrm{eff}, 2}=\mathrm{K}_{\mathrm{G}} \exp \left(\frac{\chi}{2} \sigma_{\mathrm{Y}}^{2}\right) \\
& \mathrm{K}_{\mathrm{eff}, 3}=\mathrm{K}_{\mathrm{G}} \exp \left[\left(\frac{1}{2}-\chi\right) \sigma_{\mathrm{Y}}^{2}\right]
\end{aligned}
$$

so that

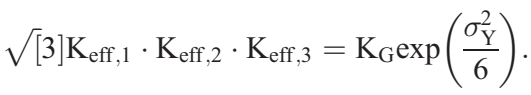

Notice that (49) does not depend on e. In the particular case of isotropy $(\mathrm{e} \rightarrow 1), \chi \rightarrow 1 / 3$ and

$$
\mathrm{K}_{\mathrm{eff}, 1}=\mathrm{K}_{\mathrm{eff}, 2}=\mathrm{K}_{\mathrm{eff}, 3}=\mathrm{K}_{\mathrm{G}} \exp \left(\frac{\sigma_{\mathrm{Y}}^{2}}{6}\right)
$$

while for $\mathrm{e} \rightarrow 0, \chi=1$ and $\mathrm{K}_{\mathrm{eff}, 1}=\mathrm{K}_{\mathrm{eff}, 2}=\mathrm{K}_{\mathrm{A}}$ and $\mathrm{K}_{\mathrm{eff}, 3}=$ $\mathrm{K}_{\mathrm{H}}$. The result is illustrated in Figure 3, depicting (48a) and (48b) as a function of $\sigma_{\mathrm{Y}}^{2}$ and e.

[56] The exponential expressions (48a) and (48b) were challenged by Indelman and Abramovich [1994a]. They first rewrote (46) in a more compact way:

$$
\mathrm{K}_{\mathrm{eff}, \mathrm{i}}=\mathrm{K}_{\mathrm{G}}\left[1+\left(\frac{1}{2}-\alpha_{\mathrm{i}}\right) \sigma_{\mathrm{Y}}^{2}\right] \quad \mathrm{i}=1,2,3,
$$

with $\alpha_{1}=\alpha_{2}=(1-\chi) / 2$ and $\alpha_{3}=\chi$. Then they found the fourth-order (in $\sigma_{\mathrm{Y}}$ ) term and wrote their final expression as

$$
\mathrm{K}_{\mathrm{eff}, i}=\mathrm{K}_{\mathrm{G}}\left\{1+\left(\frac{1}{2}-\alpha_{i}\right) \sigma_{\mathrm{Y}}^{2}+\frac{1}{2}\left[\left(\frac{1}{2}-\alpha_{i}\right)^{2}+\gamma_{i}\right] \sigma_{\mathrm{Y}}^{4}\right\} .
$$

Therefore the fourth-order term in the exponential expansion of (45a) and (45b) does not coincide with the term in (52), except for the particular cases where $\gamma_{\mathrm{i}}=0$. The $\gamma_{\mathrm{i}}$ terms were given by Indelman and Abramovich [1994a] and De Wit [1995] as a sixth-order integral in Fourier space (fourth-order in two dimensions), which they computed numerically. The $\gamma_{\mathrm{i}}$ terms vanish for $\mathrm{e}=0$ or $\mathrm{e}=1$ and reach a maximum value for $\mathrm{e} \approx 0.21$. The actual value also depends on the type of variogram selected (being highest for the Gaussian variogram). Indelman and Abramovich [1994a] and De Wit [1995] show that in two dimensions, 


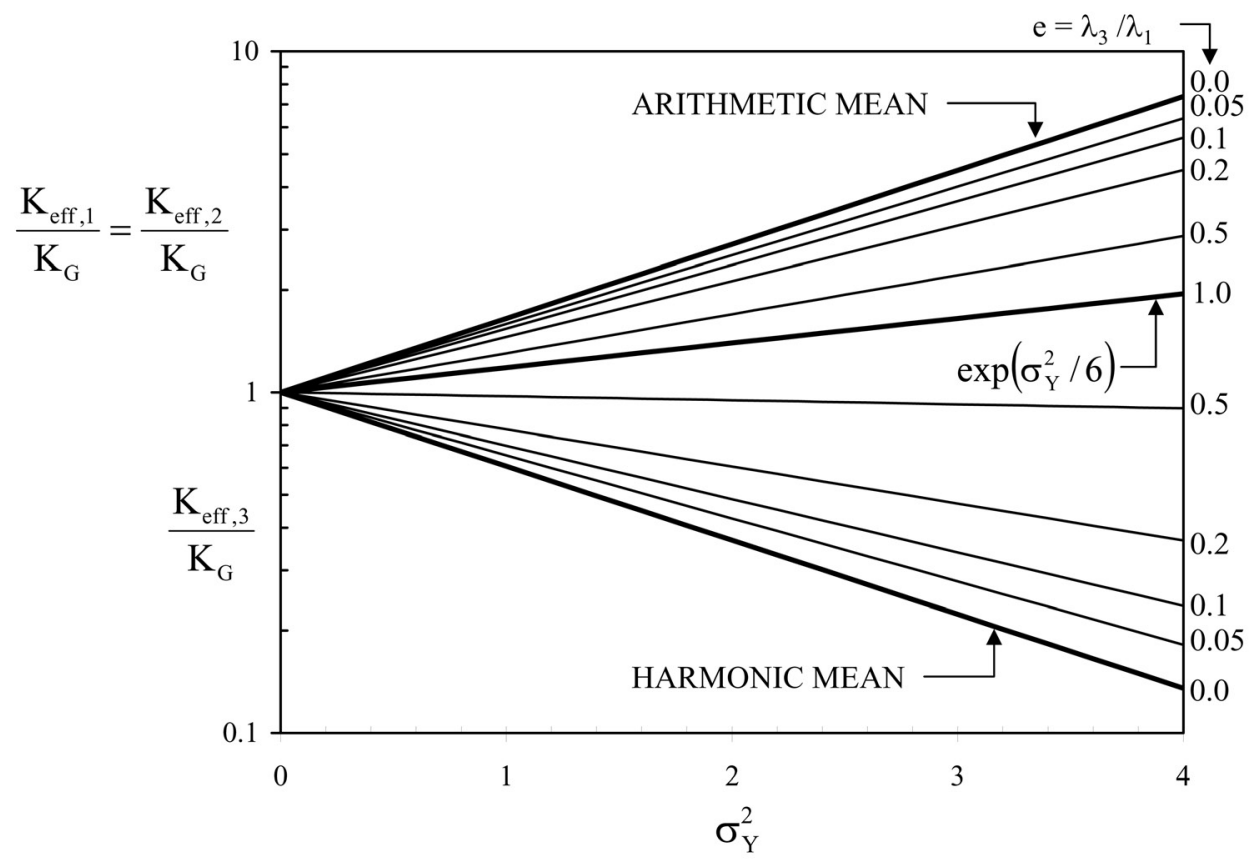

Figure 3. Dependence of normalized effective conductivities $\mathrm{K}_{\mathrm{eff}, 1} / \mathrm{K}_{\mathrm{G}}=\mathrm{K}_{\mathrm{eff}, 2} / \mathrm{K}_{\mathrm{G}}$ and $\mathrm{K}_{\mathrm{eff}, 3} / \mathrm{K}_{\mathrm{G}}$ (equations (48a) and (48b)) on $\sigma_{\mathrm{Y}}^{2}$ and e.

equation (44) is consistent with the fourth-order expansion. Later Spector and Indelman [1998] provided a simplified mathematical analysis to obtain the $\gamma_{i}$ terms in two dimensions by means of a simple integral. In particular, denoting $\gamma=-\gamma_{1}=\gamma_{2}$, the function $\gamma=\gamma(\mathrm{e})$ is provided in Figure 4. These are key results since they allow recognizing that $\mathrm{K}_{\mathrm{eff}}$ in anisotropic media depends not only on the statistical parameters of log conductivity but also on the actual shape of its correlation function.

[57] A simplified approach based on empirical arguments was suggested by Ababou [1990]. His work is based on an argument by Ababou and Wood [1990], who extended the concept of power averaging for equivalent parameters (see section 3) to ensemble power averaging. They presented a generalized solution for effective values in terms of a $p$ norm in an isotropic medium:

$$
\mathrm{K}_{\mathrm{eff}}=\left[\langle\mathrm{K}\rangle^{\mathrm{p}}\right]^{1 / \mathrm{p}}
$$

[58] When the univariate distribution of $\mathrm{K}$ is lognormal, the $\mathrm{K}_{\text {eff }}$ value defined by (53) can be expressed in terms of more commonly used averages quantities

$$
\mathrm{K}_{\mathrm{eff}}=\mathrm{K}_{\mathrm{G}} \exp \left(\mathrm{p} \sigma_{\mathrm{Y}}^{2} / 2\right)=\mathrm{K}_{\mathrm{G}}\left(\mathrm{K}_{\mathrm{G}} / \mathrm{K}_{\mathrm{H}}\right)^{\mathrm{p}}=\mathrm{K}_{\mathrm{G}}\left(\mathrm{K}_{\mathrm{A}} / \mathrm{K}_{\mathrm{G}}\right)^{\mathrm{p}} .
$$

[59] From the definition of p norm (54), Ababou [1990] conjectured an empirical value for the directional effective conductivities in anisotropic media, which is given by

$$
\mathrm{K}_{\mathrm{eff}, \mathrm{i}}=\left[\left\langle\mathrm{K}^{\mathrm{p}_{\mathrm{i}}}\right\rangle\right]^{1 / \mathrm{p}_{\mathrm{i}}} ; \quad \mathrm{p}_{\mathrm{i}}=1-\frac{2}{\mathrm{n}} \frac{\lambda_{\mathrm{H}}}{\lambda_{\mathrm{i}}},
$$

where $\lambda_{\mathrm{i}}$ is the correlation scale in the $\mathrm{i}$ direction and $\lambda_{\mathrm{H}}$ is the harmonic average of the directional correlation scales; that is,

$$
\lambda_{\mathrm{H}}=\left(\frac{1}{\mathrm{n}} \sum_{\mathrm{i}=1}^{\mathrm{n}} \lambda_{\mathrm{i}}^{-1}\right)^{-1}
$$

In the isotropic case, $\mathrm{p}=1-(2 / \mathrm{n})$, and (55) coincides with (19) in one, two, and three dimensions.

[60] In the case of two-dimensional anisotropy, (55) coincides with (44). In three dimensions the self-consistent approach in anisotropic media was used first by Poley [1988] and then by Dagan [1989] by incorporating inclu-

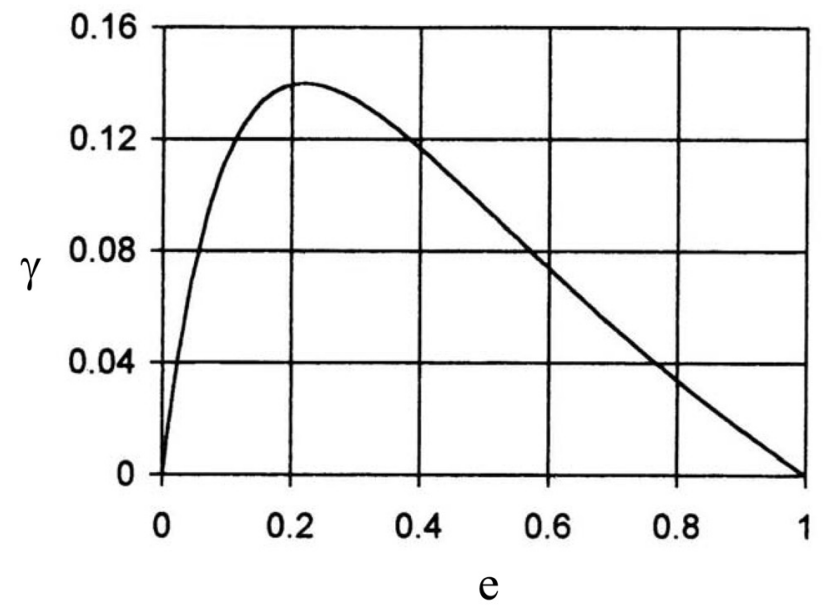

Figure 4. Function gamma as a function of e in two dimensions [from Spector and Indelman, 1998]. 


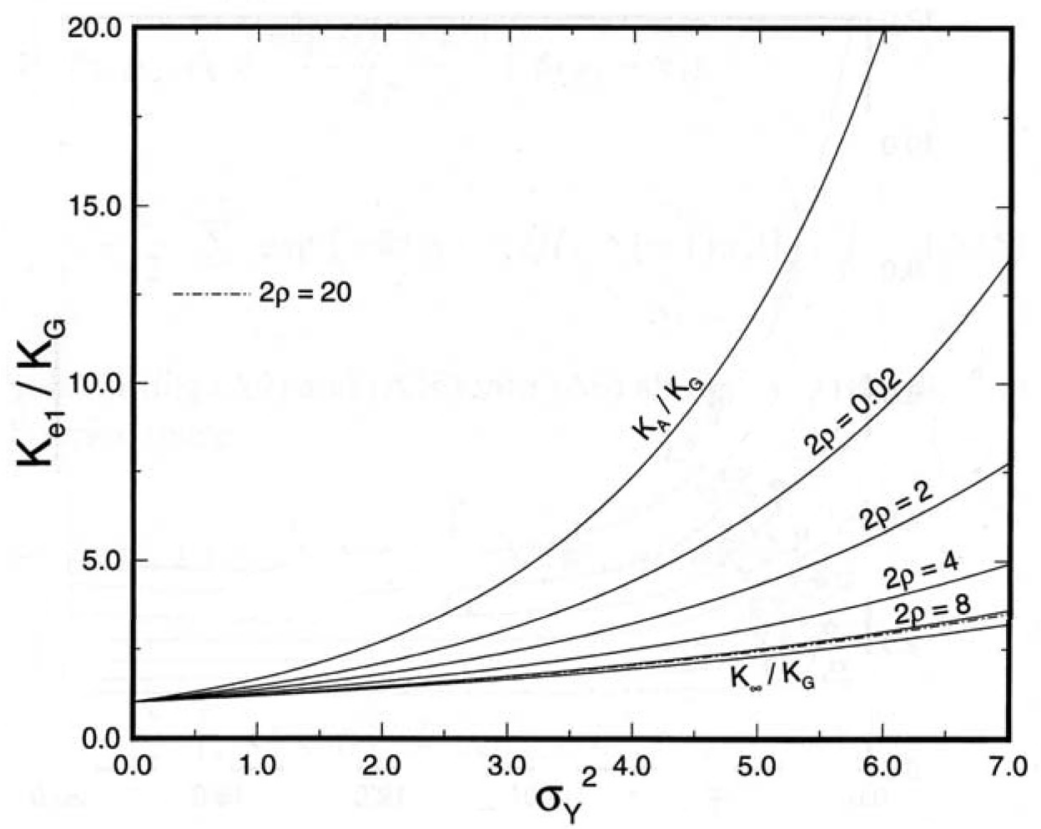

Figure 5. Generalized normalized directional effective conductivity versus $\sigma_{\mathrm{Y}}{ }^{2}$ as a function of the domain size in an isotropic three-dimensional domain. In the perpendicular direction the domain is unbounded. The term $2 \rho$ is the ratio between the total domain size and the integral distance of hydraulic conductivity [from Paleologos et al., 1996].

sions of ellipsoidal shape in the homogenized medium. Dagan [1989] provided closed-form solutions for the axisymmetric case:

$$
\begin{gathered}
\mathrm{K}_{\mathrm{eff}, 1}=\mathrm{K}_{\mathrm{eff}, 2}=\frac{1}{2}\left[\int_{0}^{\infty} \frac{\mathrm{p}(\mathrm{K})}{\left(\mathrm{K}-\mathrm{K}_{\mathrm{eff}, 1}\right) \chi\left(\mathrm{e}^{*}\right)+2 \mathrm{~K}_{\mathrm{eff}, 1}} \mathrm{dK}\right]^{-1} \\
\mathrm{~K}_{\mathrm{eff}, 3}=\left[\int_{0}^{\infty} \frac{\mathrm{p}(\mathrm{K})}{\mathrm{K}+\chi\left(\mathrm{e}^{*}\right)\left(\mathrm{K}_{\mathrm{eff}, 3}-\mathrm{K}\right)} \mathrm{dK}\right]^{-1}
\end{gathered}
$$

where the expression for $\chi$ is exactly that of (47), with $\mathrm{e}$ replaced by $\mathrm{e}^{*}=\mathrm{e}\left(\mathrm{K}_{\mathrm{eff}, 1} / \mathrm{K}_{\mathrm{eff}, 3}\right)^{1 / 2}$. This result is restricted to $\mathrm{e}<1$. The same type of approach was used by Fokker [2001] for obtaining solutions for some anisotropic two- and three-dimensional settings.

[61] So far we have considered fields where $\mathrm{K}$ was locally isotropic. Anisotropy can be caused not only by the spatial structure of heterogeneity but also by the local permeability (e.g., orientation of the grain fabric [van den Berg and de Vries, 2003]). In this case both types of anisotropy have to be superimposed. This is relevant when we compare data obtained from the field to those from samples that are taken to the laboratory and disturbed or repacked. Under these circumstances, microscale anisotropy is removed and is therefore not sampled in the laboratory tests. Since local anisotropy could be relevant for (generally reactive) solute transport process, this topic should be further explored in future research.

\subsection{Bounded Domains}

[62] Neuman and Orr [1993] analyzed the existence and properties of $\mathrm{K}_{\mathrm{eff}}$ in bounded domains, with different boundary conditions. One of the most relevant points is that in this case it is impossible to write a single value for $\mathrm{K}_{\text {eff }}$ that satisfies (4) at any given point in the domain. This will also be the case in nonuniform flow conditions, as we will see in section 4.

[63] The work of Neuman and Orr [1993] was expanded by Paleologos [1994] and Paleologos et al. [1996] for the particular case of a box-shaped domain where flow is driven by two opposing constant head faces, while the remaining boundaries are impervious. Paleologos et al. write the resulting expression as

$$
\mathrm{K}_{\mathrm{eff}}=\mathrm{K}_{\mathrm{G}} \exp \left[\left(\frac{1}{2}-\mathrm{D}\right) \sigma_{\mathrm{Y}}^{2}\right],
$$

where this expression applies for the mean flow direction. The actual expressions for D in isotropic and anisotropic domains is given by Paleologos et al. [1996]. In the general anisotropic case, D is a location-dependent quantity expressed as a triple integral that incorporates the three directional correlation scales of $\mathrm{Y}$ and the size of the domain. Figure 5 provides the effective hydraulic conductivity at the midpoint in the box (the point located farthest away from the boundaries) as a function of a normalized boundary distance. It is clear that when the 
domain is very large compared to the integral distance, the effective conductivity equals that of the unbounded domain (equation (19) or equations (48a) and (48b)). The result would tend toward the arithmetic mean for a negligible size of the domain.

[64] Equation (58) was used by Danquigny et al. [2004] to calculate the equivalent hydraulic conductivity of a heterogeneous medium reconstructed within a sand box. They used ergodic arguments and compared their obtained equivalent parameter with the effective value provided by (58). Danquigny et al. found a very good agreement, thus providing an empirical result to somehow validate the expression of Paleologos et al. [1996]. The results of Paleologos et al. [1996] are based on the simplification that when the domain size changes, the first two statistical moments of the $\mathrm{K}$ field remain constant.

[65] Tartakovsky et al. [2002] analyzed the existence of pseudoeffective parameters and their tensorial properties in a two-dimensional rectangular domain. The log conductivity field is Gaussian and exhibits an isotropic exponential correlation. Flow is driven by a given head difference between two constant head boundaries, with impervious lateral boundaries. The authors started from the work of Neuman and Orr [1993] and Guadagnini and Neuman [1999a] and, under the assumption that mean head gradient and the residual flux vary slowly in space, approximated the latter as

$$
\mathbf{r}(\mathbf{x}) \approx \mathbf{k}(\mathbf{x}) \nabla\langle\mathrm{h}(\mathbf{x})\rangle,
$$

where the tensor $\mathbf{k}$ is defined as

$$
\begin{gathered}
\mathbf{k}(\mathbf{x})=[\mathbf{I}-\mathbf{B}(\mathbf{x})]^{-1} \mathbf{A}(\mathbf{x}) \\
\mathbf{A}(\mathbf{x})=\int_{\Omega} \mathbf{a}(\mathbf{y}, \mathbf{x}) \mathrm{d} \mathbf{y}, \quad \mathbf{B}(\mathbf{x})=\int_{\Omega} \mathbf{d}(\mathbf{y}, \mathbf{x}) \mathrm{d} \mathbf{y} .
\end{gathered}
$$

These definitions lead to the following expression for the pseudoeffective conductivity:

$$
\mathbf{K}_{\text {eff }}(\mathbf{x})=\langle\mathbf{K}\rangle \mathbf{I}-\mathbf{k}(\mathbf{x}) .
$$

Tartakovsky et al. then evaluated the tensor (62) by means of a perturbation-based closure approximation. Their analytical solution evidences that the off-diagonal terms are much smaller than the diagonal ones in all cases analyzed.

[66] An additional effect of boundaries is caused by the possibility of considering random boundary conditions. Oliver and Christakos [1996] found that assuming random boundary conditions, the effective hydraulic conductivity changes near the boundary and that this effect dissipates at a distance between 2 and 4 times the integral scale depending on the covariance function. Coupling this result with Figure 5, we can argue that the actual results of Paleologos et al. [1996] are also applicable to random boundary conditions whenever the term $2 \rho$ (in Figure 5) is larger than 4.
[67] A very special case of three-dimensional bounded domains is that of an aquifer that is infinite in the horizontal directions, but not in the vertical, because of the presence of impervious boundaries (top and/or bottom of the aquifer). The first analysis was performed by Dagan [1989]. He studied the effect of one impermeable boundary. The approach is that of perturbations by using the method of images to obtain the corresponding Green's function. His main conclusion is that the presence of an impervious boundary has a very small effect on $\mathrm{K}_{\text {eff }}$ except at points located very close to the boundary.

[68] In some practical applications the variable of interest is the total flow rate integrated along the vertical. In this case it is common to introduce a parameter which is termed transmissivity, T, which can also be modeled as an SRF. In this context, Dykaar and Kitanidis [1993] evaluated two possible (intuitive) estimators: (1) $\mathrm{K}_{\text {eff }}$ times the aquifer thickness and (2) an effective transmissivity within a threedimensional domain given by the geometric mean of the vertically integrated $\mathrm{K}$ values. They found that both quantities overestimate the $T_{\text {eff }}$ value obtained by numerical simulations. Tartakovsky et al. [2000] found the actual expression for $T_{\text {eff }}$ in terms of the statistical moments of $\log$ conductivity, the vertical aquifer thickness, B, and the vertical correlation scale, $\lambda_{3}$, of $\log$ conductivity:

$$
\begin{gathered}
\mathrm{T}_{\text {eff }}=\mathrm{BK}_{\mathrm{G}}\left(1+\frac{1-\beta}{2} \sigma_{\mathrm{Y}}^{2}\right) \\
\beta=\lambda_{3}^{2}\left[\sqrt{\pi} \lambda_{3}^{-1} \operatorname{erf}\left(\lambda_{3}^{-1}\right)+\exp \left(-\lambda_{3}^{-2}\right)-1\right] .
\end{gathered}
$$

Here $\beta\left(\lambda_{3}\right)$ is a monotonic increasing function, ranging between $\beta=0$ for $\lambda_{3} \rightarrow 0$ and $\beta=1$ for $\lambda_{3} \rightarrow \infty$, leading to the limiting values $\mathrm{T}_{\text {eff }}=\mathrm{BK}_{\mathrm{A}}$ and $\mathrm{T}_{\text {eff }}=\mathrm{BK}_{\mathrm{G}}$ for $\lambda_{3} \rightarrow 0$ and $\lambda_{3} \rightarrow \infty$, respectively. The result is depicted in Figure 6, which shows the dependence of $\mathrm{T}_{\mathrm{eff}}$ on $\sigma_{\mathrm{Y}}^{2}$ and $\lambda_{3}$.

\subsection{Numerical Solutions: Monte Carlo Approach}

[69] The Monte Carlo method is conceptually simple and needs no particular assumptions. This method has been widely used in the literature of effective parameters since it allows assessing the accuracy of low-order approximations of the kind we explored in sections 2.1, 2.2, and 2.3 and analyzing general complex scenarios that are not amenable to direct analytical solutions. In the Monte Carlo method one starts by assuming that the multivariate probability distribution of the input variable, $\mathbf{K}(\mathbf{x}, \omega)$, is fully known. Equally likely hydraulic conductivity fields (termed realizations) are synthetically generated. The flow equation with appropriate boundary conditions is then solved for each realization. Finally, statistics of model outputs are computed. Specifically, it is possible to obtain numerically both $\langle\mathbf{q}\rangle$ and $\nabla\langle\mathrm{h}\rangle$, so that an effective or pseudoeffective hydraulic conductivity can be computed. On the other hand, a key theoretical disadvantage of the Monte Carlo method is that it does not allow us to rigorously prove the existence of such parameters. 


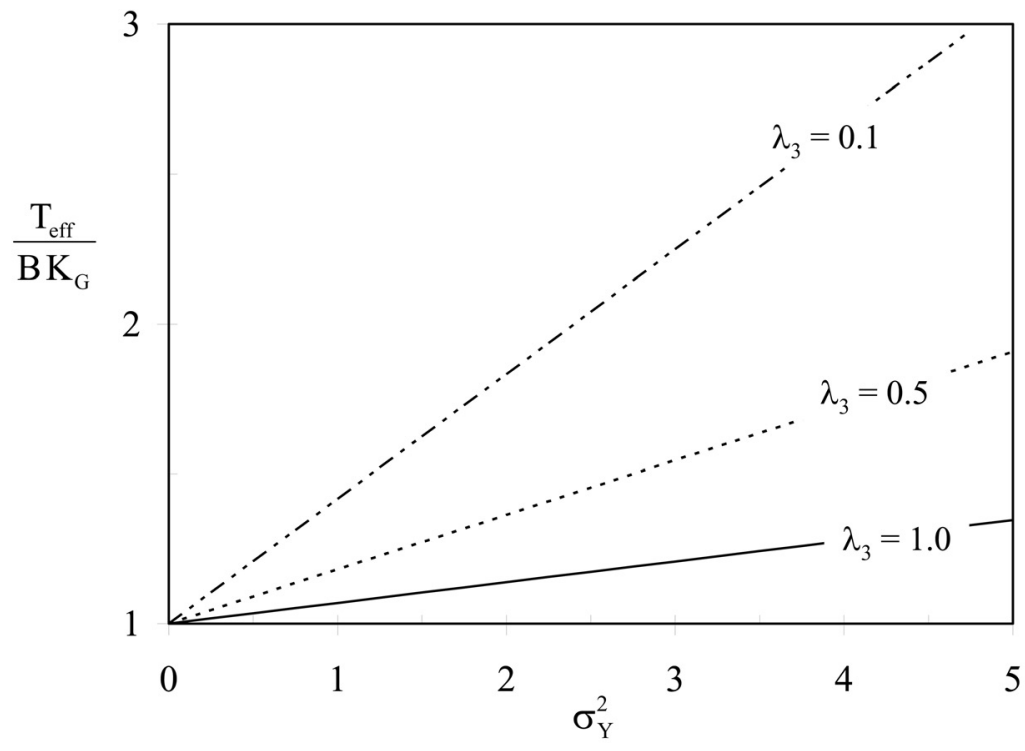

Figure 6. Dependence of $\mathrm{T}_{\mathrm{eff}} /\left(\mathrm{BK}_{\mathrm{G}}\right)$ (equation (63)) on $\sigma_{\mathrm{Y}}^{2}$ and $\lambda_{3}$.

[70] One of the critical points in a Monte Carlo analysis is the generation of a large number of conditional realizations of the input random field given the multivariate pdf of the SRF. Several methods are now available in the geostatistical literature. Without being exhaustive some of the existing generation methods are the following: the turning bands [Mantoglou and Wilson, 1982]; the nearest-neighbor method [Smith and Freeze, 1979]; the matrix-decomposition method [Clifton and Neuman, 1982]; the sequential simulation, both for multi-Gaussian [Gómez-Hernández, 1991] and indicator fields [Gómez-Hernández and Srivastava, 1990]; the probability field simulation [Froidevaux, 1993]; the truncated Gaussian method [Matheron et al., 1987]; Boolean-type methods [e.g., Jussel et al., 1994a] as well as other structure-imitating methods [e.g., Koltermann and Gorelick, 1996; Deutsch and Tran, 2002]; simulated annealing [Deutsch and Journel, 1992; Deutsch and Cockerham, 1994]; and process-imitating methods [see Koltermann and Gorelick, 1996 for a review], as well as other genetictype methods [e.g., Webb and Anderson, 1996; Teles et al., 2004].

[71] Freeze [1975] first applied the Monte Carlo method in the context of one-dimensional flow to find the ensemble head distribution, therefore being able to check numerically the result for effective hydraulic conductivity. The first simulations in two and three dimensions specifically used to analyze effective conductivity values were carried out by Smith and Freeze [1979] and Desbarats [1992], respectively. It is important to stress that numerical simulations are always carried out in finite domains. Despite that, in many cases the results are analyzed as if corresponding to infinite domains. This simplification holds whenever the domain is very large with respect to the corresponding directional integral scales of $\mathrm{Y}$ or $\mathrm{K}$.

[72] There are many interesting applications of the Monte Carlo approach to compute effective conductivity values.
Follin [1992] was able to confirm numerically the validity of $\mathrm{K}_{\mathrm{eff}}=\mathrm{K}_{\mathrm{G}}$ in a two-dimensional infinite domain up to at least $\sigma_{\mathrm{Y}}^{2}=16$. Dykaar and Kitanidis [1992] were able to confirm the three-dimensional expression for unbounded domains given in (19) up to $\sigma_{\mathrm{Y}}^{2}=6$.

[73] In uncorrelated media, Shrestha and Loganathan [1994] confirmed the results of Freeze [1975] for onedimensional steady state saturated flow. In anisotropic media, Naff et al. [1998] tested the validity of (46a). They found quite a good agreement between theory and the numerical solution for $\sigma_{\mathrm{Y}}^{2}<1$. They also found that the term in $\sigma_{\mathrm{Y}}^{4}$ was practically irrelevant, so that an approximation up to the order of $\sigma_{\mathrm{Y}}^{2}$ was sufficiently accurate for the range of variances tested. Revelli and Ridolfi [2000] applied the Monte Carlo method to find effective values in unconfined aquifers and found that for increasing $\sigma_{\mathrm{Y}}^{2}$ values, there is a very slight increase in the $\mathrm{K}_{\text {eff }}$ value when the nonlinearity associated with unconfinement is incorporated into the numerical model. The Monte Carlo approach has also been used to compute numerically effective conductivities in bimodal fields [e.g., McCarthy, 1990].

\subsection{Transient Flow}

[74] The initial works on transient flow were carried out by Alonso and Krizek [1974] and Freeze [1975] in onedimensional flow to study the particular problem of consolidation. In those works, hydraulic conductivity was considered random but with a negligible correlation distance. Dagan [1982] was the first author to incorporate nonnegligible correlation distances in the analysis of transient flow. He analyzed both the cases of transient flow with average head gradients slowly varying in time and periodic flow. In the former case, and from a perturbations expansion approach, an effective hydraulic conductivity $\mathrm{K}_{\text {eff }}(\mathrm{t})$ can be defined as a function of time, $\mathrm{t}$. For short times, $\mathrm{K}_{\text {eff }}$ is equal to the arithmetic mean, $\mathrm{K}_{\mathrm{A}}$, and relaxes gradually with time 
to the steady state value, $\mathrm{K}_{\text {eff,ss }}$. The actual solution for an infinite domain can be written as

$$
\begin{gathered}
\frac{\mathrm{K}_{\text {eff }}(\mathrm{t})-\mathrm{K}_{\text {eff }, \text { ss }}}{\mathrm{K}_{\mathrm{A}}-\mathrm{K}_{\text {eff }, \mathrm{ss}}}=\mathrm{b}(\mathrm{t}) \\
\mathrm{b}(\mathrm{t})=\frac{1}{\sigma_{\mathrm{Y}}^{2}} \int \mathrm{C}_{\mathrm{Y}}\left(\mathbf{x}-\mathbf{x}^{\prime}\right) \mathrm{G}\left(\mathbf{x}, \mathbf{x}^{\prime}, \mathrm{t}\right) \mathrm{d} \mathbf{x}^{\prime} .
\end{gathered}
$$

Function $b(t)$ incorporates the covariance function of $Y, C_{Y}$ and the transient Green's function. Dagan [1982] provided the actual closed-form expression for (66) in the case of an exponential covariance function:

$$
b(t)=\left\{\begin{array}{cc}
\exp \left(t^{*}\right) \operatorname{erfc}\left(\sqrt{t^{*}}\right) & \text { in one dimension } \\
1-\sqrt{\pi t^{*}} \exp \left(t^{*}\right) \operatorname{erfc}\left(\sqrt{t^{*}}\right) & \text { in two dimensions } \\
-2 \sqrt{t^{*} / p}+\left(1+2 t^{*}\right) \exp \left(t^{*}\right) \operatorname{erfc}\left(\sqrt{t^{*}}\right) & \text { in three dimensions }
\end{array}\right.
$$

$$
\mathrm{t}^{*}=\frac{\mathrm{K}_{\mathrm{G}} \mathrm{t}}{\langle\mathrm{S}\rangle \mathrm{I}_{\mathrm{Y}}^{2}},
$$

where $I_{Y}$ is the integral scale of $Y$ and $S$ is storativity, which is also modeled as a correlated SRF. The relaxation time, defined as the time where $\mathrm{K}_{\text {eff }}(\mathrm{t})$ approaches its asymptotic value, equivalent to that of the steady state case for the same boundary conditions, is much larger for one-dimensional flow than for two- and three-dimensional flows. Another important point is that the correlation between $\log$ conductivity and storativity does not influence the value of $\mathrm{K}_{\text {eff }}$ at the leading order. Further discussion about the relaxation time values for these problems is given by Dagan [1989]. El-Kadi and Brutsaert [1985] conducted numerical Monte Carlo simulations of unsteady gravity drainage from a large unconfined aquifer to analyze the applicability of effective parameters under general nonuniform flow conditions in heterogeneous aquifers. Their main result is to stress the importance of properly accounting for boundary conditions in the evaluation of transient effective parameters.

[75] Indelman [1996] derived expressions for the transient effective conductivity in the Fourier-Laplace space for the case of unbounded domains of statistically homogeneous $\log$ conductivity and showed that the effective conductivity is a local property of the medium in the Fourier-Laplace space. This locality is lost when the governing equation is written in physical space, so that a pseudoeffective conductivity does not exist in general. This result calls into question the general applicability of (65).

[76] Tartakovsky and Neuman [1998a] use the residual flux theory to obtain an approximation to second order (in $\sigma_{\mathrm{Y}}$ ) for a transient effective conductivity that is nonlocal in time but local in space:

$$
\begin{gathered}
\langle\mathbf{q}(\mathbf{x}, \mathrm{t})\rangle=-\mathbf{K}_{\mathrm{G}}\left(1+\frac{\sigma_{\mathrm{Y}}^{2}}{2}\right) \nabla\langle\mathrm{h}(\mathrm{t})\rangle+\int_{0}^{\mathrm{t}} \kappa^{*}(\mathbf{x}, \mathrm{t}-\tau) \nabla\langle\mathrm{h}(\tau)\rangle \mathrm{d} \tau \\
\kappa^{*}(\mathbf{x}, \mathrm{t}-\tau)=\mathrm{K}_{\mathrm{G}}^{2} \int_{\mathrm{V}} \mathrm{C}_{\mathrm{Y}}\left(\mathbf{x}-\mathbf{x}^{\prime}\right) \nabla_{\mathbf{x}} \nabla_{\mathbf{x}^{\prime}}^{\mathrm{t}} \mathrm{G}\left(\mathbf{x}^{\prime}, \mathbf{x}, \mathrm{t}-\tau\right) \mathrm{d} \mathbf{x}^{\prime} .
\end{gathered}
$$

To arrive at these expressions, the authors first localized the exact average equations under the assumption that mean flow and residual flux vary slowly in space and time and then used perturbations. As in the work of Indelman [1996], Tartakovsky and Neuman found that the transient effective conductivity is local in Fourier-Laplace space. The solution of Tartakovsky and Neuman [1998b] for infinite domain and gradually varying flow coincides with that of Dagan [1982]. One of the advantages of their methodology is that it can be used for bounded domains.

[77] One must not confuse transient effective conductivity values caused by the transient effects of head gradients with the variability in the steady state values with time, $\mathrm{K}_{\text {eff,ss }}(\mathrm{t})$, because of other causes such as variation of the pdf of $\mathrm{K}$ with time. One such example is provided by Whitaker and Smart [1997] where effective conductivity is associated with enhanced dissolution of carbonates and therefore correlated with rainfall and evolving in time.

\subsection{Nonstationary Conductivity Fields}

[78] There are several possibilities for nonstationarity in the SRF function, $\mathbf{K}(\mathbf{x}, \omega)$. In this section we will address three different topics. Nonstationarity may arise because of the presence of space variations in mean conductivity. It might also arise from a variogram displaying no sill (unbounded variance). Finally, we will also address the case where second-order moments are not sufficient to fully characterize the random $\mathrm{K}$ field, i.e., when $\mathrm{K}$ processes are not multi-Gaussian. We do not analyze here the case of spatially variable variance and autocovariance functions, which may emerge from conditioning the (log) conductivity field to point measurements of $\mathrm{K}$. This topic will be discussed in section 5. Nonstationarity usually generates nonuniform mean flow conditions, so we cannot generally use the results presented so far.

\subsubsection{Mean Conductivity Variable in Space}

[79] This is another case where it is impossible to define effective values, as, logically, they must depend on position. Therefore we would talk here about pseudoeffective hydraulic conductivities which can be interpreted as parameters derived by a localization of the exact (or approximated) mean flow equations. Gelhar [1993] analyzed the case of trending media by means of perturbation and spectral analysis. For a three-dimensional isotropic case, with an exponential correlation function and in the case of a trend (in mean conductivity) only in the direction of the mean gradient, he found the following compact expression:

$$
\mathrm{K}_{\mathrm{eff}}(\mathrm{x})=\mathrm{K}_{\mathrm{G}}\left[1+\frac{\sigma_{\mathrm{Y}}^{2}}{6}+\frac{\sigma_{\mathrm{Y}}^{2}(\mathrm{~A} \lambda)^{2}}{5}\right]
$$

valid for $\mathrm{A} \lambda \ll 1$. Here $\lambda$ is the (isotropic) correlation scale of $\log$ conductivity, and A is a trend parameter, which is considered to be small but finite and is interpreted as proportional to a typical scale of nonuniformity in the mean hydraulic gradient. The author then shows that use of the spectral density function of log conductivity proposed by 
Mizell et al. [1982] leads to the following expression in the case of two-dimensional media:

$$
\mathrm{K}_{\mathrm{eff}}(\mathrm{x})=\mathrm{K}_{\mathrm{g}}\left[1+\frac{3}{8}\left(\frac{\mathrm{A}}{\alpha}\right)^{2} \sigma_{\mathrm{Y}}^{2}\right], \quad \frac{\mathrm{A}}{\alpha} \ll 1, \quad \alpha=\frac{1.045}{\lambda_{\mathrm{e}}},
$$

where $\lambda_{\mathrm{e}}$ is the separation distance at which correlation decreases to $\exp (-1)$. Equations (70) and (71) allow one to observe that a trend in hydraulic conductivity will generally produce an increase in the pseudoeffective conductivity along the direction of the trend.

[80] Probably the first analysis of pseudoeffective hydraulic conductivity in the presence of a trending mean hydraulic conductivity was performed by Loaiciga et al. [1993]. They carry out a perturbations expansion to first order in $\sigma_{\mathrm{Y}}$ using the spectral method. With our notation their expression for $\mathrm{K}_{\mathrm{eff}}$ in one-dimensional space can be expressed as

$$
\mathrm{K}_{\mathrm{eff}}(\mathrm{x})=\exp (\mathrm{m}(\mathrm{x}))\left[1-\frac{\sigma_{\mathrm{Y}}^{2}}{\beta \lambda+1}\right]
$$

where $\mathrm{m}$ is the (deterministic) trend of $\langle\mathrm{Y}\rangle$, which is a function of $\mathrm{x}$, and $\beta=\mathrm{dm} / \mathrm{dx}$ (assuming $\mathrm{m}$ is a continuous and differentiable function). In the equation it is specifically noted that $\mathrm{K}_{\text {eff }}$ is a function of location. Owing to the firstorder approximation this result does not provide the proper solution, $\mathrm{K}_{\mathrm{eff}}=\mathrm{K}_{\mathrm{G}}$, for $\mathrm{m}=0$. Loaiciga et al. [1993] provided an expression in integral form for $\mathrm{K}_{\text {eff }}$ in three dimensions. The main result is that $\mathrm{K}_{\text {eff }}$ is necessarily anisotropic. Later, Loaiciga et al. [1994, 1996] completed the work by providing solutions for two and three dimensions and found a solution of the type

$$
\mathrm{K}_{\mathrm{eff}}(\mathbf{x})=\exp [\mathrm{m}(\mathbf{x})]\left[1-\sigma_{\mathrm{Y}}^{2} \mathrm{~F}(\beta, \mathrm{I}, \theta)\right]
$$

where $\theta$ is the angle between hydraulic gradient and the direction of the trend.

[81] A second-order (in $\sigma_{\mathrm{Y}}$ ) perturbation expansion was used by Rubin and Seong [1994] to find effective hydraulic conductivity when the mean hydraulic gradient is either parallel or perpendicular to the trend in mean log conductivity. They wrote their results in a compact expression

$$
\mathrm{K}_{\mathrm{eff}}(\mathbf{x})=\mathrm{K}_{\mathrm{G}}(\mathbf{x})\left(1+\sigma_{\mathrm{Y}}^{2}\left(\frac{1}{2}-\mathrm{A}\right)\right),
$$

where the actual expression for $\mathrm{A}$ is an integral in the spectral space that depends on whether the direction of the trend is parallel to the mean hydraulic gradient. In this case their expression reduces to

$$
\mathrm{A}=\frac{1}{2 \pi} \int_{-\infty}^{+\infty} \hat{\rho}_{\mathrm{Y}}(\mathbf{k}) \frac{\mathrm{k}_{1}^{4}+\mathrm{k}_{1}^{2} \mathrm{k}_{2}^{2}+\beta^{2} \mathrm{k}_{1}^{4}}{\mathrm{k}^{4}+\beta^{2} \mathrm{k}_{1}^{2}} \mathrm{~d} \mathbf{k}
$$

where $\mathbf{k}$ is a coordinate vector of components $\left(\mathrm{k}_{1}, \mathrm{k}_{2}\right)$ in Fourier space and $\hat{\rho}_{\mathrm{Y}}(\mathbf{k})$ is the Fourier transform of the correlation function of the log conductivity. Alternatively, when they are perpendicular,

$$
\mathrm{A}=\frac{1}{2 \pi} \int_{-\infty}^{+\infty} \hat{\rho}_{\mathrm{Y}}(\mathbf{k}) \frac{\mathrm{k}_{1}^{4}+\mathrm{k}_{1}^{2} \mathrm{k}_{2}^{2}}{\mathrm{k}^{4}+\beta^{2} \mathrm{k}_{2}^{2}} \mathrm{~d} \mathbf{k}
$$

This result is depicted in Figure 7, which shows (74) for both cases (trend normal or parallel to the mean hydraulic head gradient).

[82] Their work was extended by Indelman and Rubin [1995, 1996], who provided a solution for any given angle between trend and mean gradient in the case of a linear trend in the mean $\log$ conductivity, i.e., $\mathrm{K}(\mathbf{x})=\mathrm{K}_{\mathrm{G}}(\mathbf{x}=0)$ $\exp \left(\boldsymbol{\lambda} \cdot \mathbf{x}+\mathrm{Y}^{\prime}(\mathbf{x})\right)$, which allows recovering the following consistent second-order (in $\sigma_{\mathrm{Y}}$ ) expansion:

$$
\begin{aligned}
& \mathrm{K}_{\mathrm{eff}, \mathrm{ij}}(\mathbf{x})=\mathrm{K}_{\mathrm{G}}(\mathbf{x})\left(\delta_{\mathrm{ij}}+\sigma_{\mathrm{Y}}^{2}\left(\frac{1}{2} \delta_{\mathrm{ij}}-\alpha_{\mathrm{ij}}\right)\right), \quad(\mathrm{i}, \mathrm{j}=1,2) \\
& \alpha_{\mathrm{ij}}=\frac{1}{(2 \pi)^{\mathrm{D}}} \int_{-\infty}^{+\infty} \hat{\rho}_{\mathrm{Y}}(\mathbf{k}) \frac{\left(\mathrm{k}_{\mathrm{i}}+i \gamma_{\mathrm{i}}\right) \mathrm{k}_{\mathrm{j}}}{\mathrm{k}^{2}-2 i \chi \cdot \mathbf{k}} \mathrm{dk}, \quad \gamma=\frac{\boldsymbol{\lambda} \cdot \mathbf{J}}{\mathrm{J}^{2}} \mathbf{J}, \\
& \chi=1 / 2 \boldsymbol{\lambda}-\gamma .
\end{aligned}
$$

Here $\mathrm{D}$ is the number of space dimensions and $i$ and $\mathbf{J}$ are the imaginary unit and the mean head gradient, respectively.

[83] A different methodology for obtaining pseudoeffective parameters in a trending medium was proposed by $\mathrm{Li}$ and McLaughlin [1995]. The methodology is an extension of the spectral method to express a nonstationary stochastic process as an integral in Fourier space in terms of random Fourier increments. One advantage is that it allows incorporating any type of trend in mean $\mathrm{K}$ (not necessarily linear or exponential) and also boundaries. $\mathrm{Li}$ and McLaughlin applied the method to the case of infinite domain, exponential trend, and mean gradient parallel to the trend and recovered results which are similar to those of Indelman and Rubin [1995].

\subsubsection{Nonstationary Variogram}

[84] Here we consider the effect of nonstationary behavior described in terms of power law semivariogram or, in other words, by conceptualizing the (log) hydraulic conductivity as a self-affine fractal. We would rather not focus either on the motivations leading to a multifractal representation of nature or on the underlying theory. An excellent review of these topics has been recently provided by Neuman and Di Federico [2003]. Our aim is to present the implication of this conceptual framework on the existence and actual expressions for the effective and pseudoeffective hydraulic conductivity.

[85] Neuman [1994] analyzed the effect of the nonstationary structure of a random (log) permeability field on the effective conductivity. He started from the premise that the spatial heterogeneity of many geologic media can be described by treating $\log$ conductivity as a multiscale random field defined over a continuum, so that such a field can be modeled by a power law semivariogram. Following 


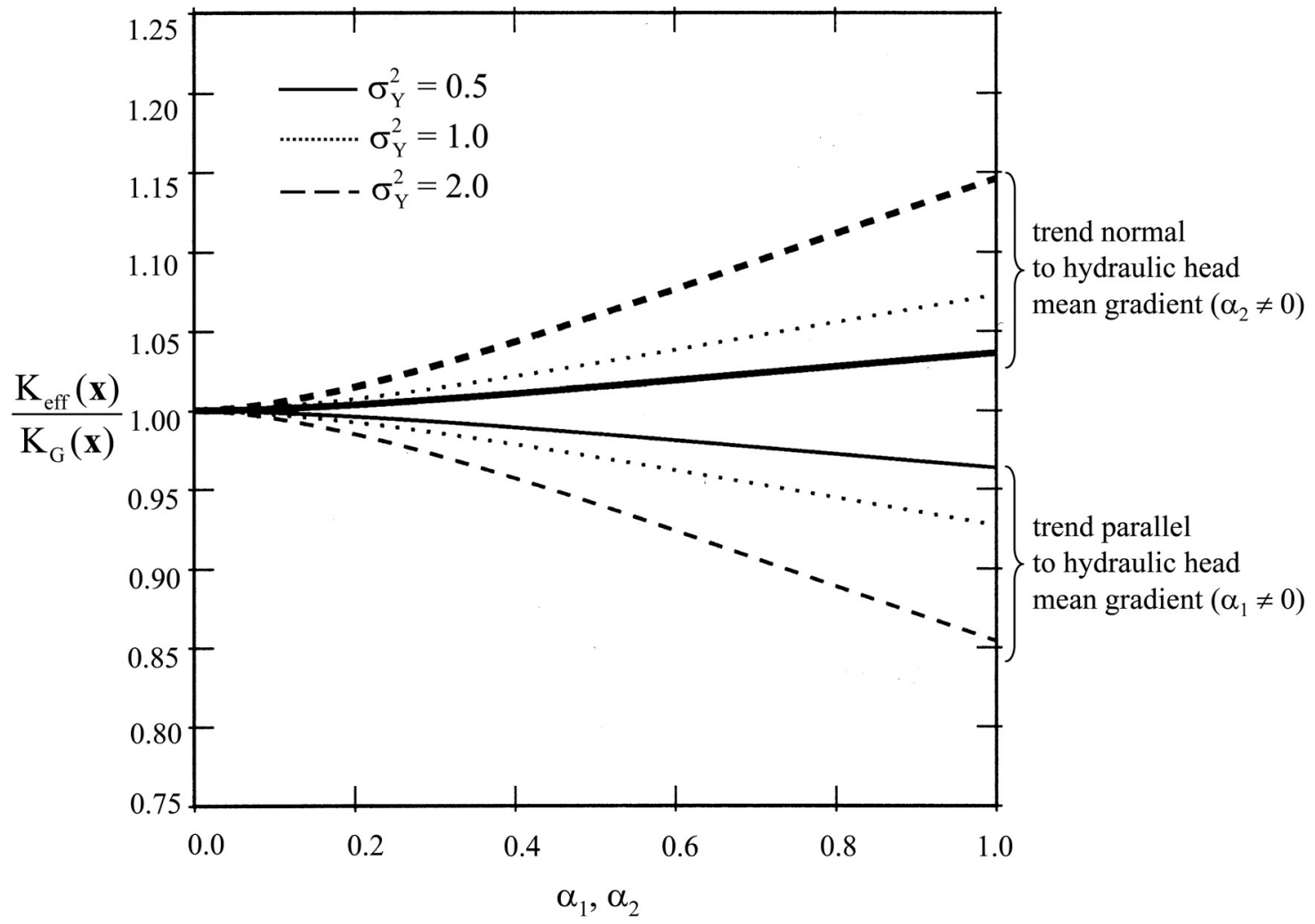

Figure 7. Normalized pseudoeffective hydraulic conductivity (equation (74)) for a trending medium. The expected value of log conductivity, $\langle\mathrm{Y}(\mathbf{x})\rangle$, depends on the space coordinate, $\mathbf{x}$, as $\langle\mathrm{Y}(\mathbf{x})\rangle=\mathrm{m}_{0}+\boldsymbol{\alpha}$ $\mathbf{x}$, where $\mathrm{m}_{0}$ and vector $\boldsymbol{\alpha}=\alpha_{\mathrm{i}}(\mathrm{i}=1,2, \ldots, \mathrm{n}, \mathrm{n}$ being space dimensionality) are constants. Space coordinate and $\alpha$ are normalized with respect to the log conductivity integral scale. Adapted from Rubin and Seong [1994].

Neuman [1990], he then noted that a log conductivity field characterized by a semivariogram $\gamma(\mathrm{s})=\mathrm{A}_{0} \mathrm{~s}^{2 \mathrm{H}}$ (where $\mathrm{s}$ is distance, $\mathrm{A}_{0}$ is a constant, and $\mathrm{H}$ is the Hurst coefficient) can be conceptualized as an infinite hierarchy of mutually uncorrelated statistically homogeneous random fields with exponential semivariograms (for $\mathrm{H} \leq 1 / 2$ ). He then investigated theoretically the implications of such power law behavior on the expression of the effective hydraulic conductivity of a medium sample of finite size, L, under mean uniform flow conditions,

$$
\mathrm{K}_{\mathrm{eff}}(\mathrm{L})=\mathrm{K}_{\mathrm{G}} \exp \left[\mathrm{A}_{0} \mathrm{~L}^{2 \mathrm{H}}\left(\frac{1}{2}-\frac{\beta}{\mathrm{n}}\right)\right],
$$

where $0 \leq \beta \leq \mathrm{n}$, somehow describing the degree of medium anisotropy. In essence, Neuman [1994] derived (78) as an extension of the results for stationary anisotropic random fields just by replacing the variance in those expressions by $\mathrm{A}_{0} \mathrm{~L}^{2 \mathrm{H}}$. From (78) it is clear that in isotropic fields $(\beta \approx 1)$ the effective conductivity should decrease with $\mathrm{L}$ in one dimension, does not depend on $\mathrm{L}$ in two dimensions, and increases with $\mathrm{L}$ in three dimensions.

[86] Beckie et al. [1994] studied the interaction between subgrid and resolved scales when solving Darcy's flow regimes. They concluded that a nonlocal form of Darcy's law is a more appropriate descriptor of the medium behavior when hydraulic conductivity is characterized by many scales of variability. In this case they demonstrated numerically that errors derived by the use of a local form of Darcy's law with an effective parameter are most relevant at the smallest resolved scales of the model.

[87] Di Federico et al. [1999] adopted the conceptual framework of Neuman [1994] and developed an expression for the pseudoeffective (the authors refer to it as "effective") conductivity of a box-shaped porous medium. The $\log$ conductivity field is modeled as a statistically anisotropic fractional Brownian motion [e.g., Mandelbrot and Van Ness, 1968]. The box is oriented parallel to the principal coordinates of statistical anisotropy, and flow is driven by two constant head boundaries between lateral noflow boundaries. Starting from (61), they derived the following second-order expression

$$
\mathrm{K}_{\text {eff }}\left(\mathbf{x} ; \mathrm{H}, \lambda_{\mathrm{l}}\right)=\mathrm{K}_{\mathrm{G}}\left[1+\sigma^{2}\left(\mathrm{H}, \lambda_{\mathrm{l}}\right)\left(\frac{1}{2}-\mathrm{D}_{\mathrm{st}}(\mathbf{x} ; \mathrm{H})\right)\right]
$$

where $\lambda_{1}$ is a low-frequency cutoff, $\sigma^{2}\left(\mathrm{H}, \lambda_{l}\right)=A \lambda_{l}^{2 \mathrm{H}} / 2 \mathrm{H}$, and the function $\mathrm{D}_{\text {st }}$ is given by equation (51) of $D i$ Federico et al. [1999]. A generalization of (79) to higher order in $\sigma^{2}$ is

$$
\mathrm{K}_{\mathrm{eff}}\left(\mathbf{x} ; \mathrm{H}, \lambda_{\mathrm{l}}\right)=\mathrm{K}_{\mathrm{G}} \exp \left\{\sigma^{2}\left(\mathrm{H}, \lambda_{\mathrm{l}}\right)\left[\frac{1}{2}-\mathrm{D}_{\mathrm{st}}(\mathbf{x} ; \mathrm{H})\right]\right\} .
$$


The result of Di Federico et al. [1999] offers a quite unique perspective. First, it applies to a continuum of scales. Then, it implies that the natural logarithm of $\mathrm{K}_{\text {eff }}, \mathrm{Y}_{\text {eff }}$, is given by the sum of two components, respectively accounting for unresolved fluctuations at scales larger and smaller than those of a grid block represented by their investigated domain. This suggests that (in principle) an effective property can be interpreted as a random quantity, and Di Federico et al. complemented (80) with expressions for the variance-covariance of effective values. Neuman and $D i$ Federico [2003] then showed evidence that (1) averaging (80) over the box yields lower and upper effective conductivities independent of $\mathbf{x}$ and (2) the box-scale effective conductivity for a superimposed multiscale hierarchy of modes increases with the box size.

\subsubsection{Non-multi-Gaussian Fields}

[88] Most of the results presented so far are based on statistical distributions of the conductivity field, which are an extension of the normal or lognormal univariate distribution to the multivariate one (multi-Gaussian or multilogGaussian). The main advantage of this type of model is that the first-order (mean) and the second-order (covariance function) moments are enough to fully characterize the spatial random function K. Journel and Deutsch [1993] state that the multi-Gaussian model is a maximum entropy model, i.e., a model that minimizes unwarranted structural properties and maximizes spatial disorder. For this reason, high conductivity values (or low conductivity ones) are least connected using a multi-Gaussian model.

[89] As shown, for example, by Koltermann and Gorelick [1996] or Teles et al. [2004], real geological structures are seldom multi-Gaussian but rather present some connected paths of high or low conductivity values that are relevant to groundwater flow and solute transport. Sanchez-Vila et al. [1996] showed the importance of connectivity of high K values in groundwater flow, while Gómez-Hernández and Wen [1998] showed the impact of the multivariate distribution choice on traveltime estimations. Connectivity issues are also important in environmental applications such as leachate evolution from landfill sites or seawater intrusion [Nobes, 1996].

[90] Most of the studies in effective conductivity for nonmulti-Gaussian random fields have been carried out numerically. Initial work was presented by Silliman and Wright [1988], who focused on the possibility of having continuous paths of high hydraulic conductivity values and studied how this could affect groundwater flow in the subsurface. Another relevant work is that of Jussel et al. [1994b]. They created a numerical simulation using a Boolean approach to simulate a highly heterogeneous gravel deposit mimicking field-based observations. They obtained an effective conductivity that was higher than the value that would correspond to a multi-Gaussian field and associated this result with numerical errors and not with the non-multi-Gaussian nature of the field.

[91] As mentioned above, one of the effects of multiGaussianity is that extreme values are poorly connected. Sanchez-Vila et al. [1996] evaluated the effective hydraulic conductivity of two-dimensional domains where this connectivity between high $\mathrm{K}$ values was artificially enhanced. They found a consistent deviation from (19), so that in all cases analyzed $\mathrm{K}_{\mathrm{eff}}>\mathrm{K}_{\mathrm{G}}$ in all directions. Similar results were obtained by Zinn and Harvey [2003]. This result does not contradict (19), as the latter equation was obtained under some restrictive hypotheses upon the spatial laws characterizing $\mathrm{K}(\mathbf{x}, \omega)$ and $\mathrm{K}^{-1}(\mathbf{x}, \omega)$. These restrictions are not necessarily met in non-multi-Gaussian log $\mathrm{K}$ fields.

[92] Pickup et al. [1994] and Pickup and Hern [2002] studied, again numerically, effective hydraulic conductivity in a two-dimensional model of cross-bedded sandstones derived from pictures coming from outcrops. They found different values for the $\mathbf{K}_{\text {eff }}$ tensor depending upon whether laminae display a low or a high angle and whether spacing between laminae is wide or not. They found that close spacing led to lower $\mathrm{K}_{\mathrm{eff}, \mathrm{ij}}$ values and that the off-diagonal term in the tensor was comparable to the smallest principal $\mathrm{K}_{\mathrm{eff}, \mathrm{j}}$ value.

[93] A way to overcome the problem of finding analytical effective values in non-multi-Gaussian fields was carried out by Poeter and Townsend [1994]. They studied a twodimensional section of a braided fluvial aquifer with enhanced connectivity. They started by finding the paths with the highest percent of high hydraulic conductivity hydrofacies and obtained an analytical approximation of the effective conductivity by computing the harmonic average of the conductivities along those paths.

\subsection{Bimodal Fields}

[94] A bimodal distribution models the medium as if it were composed of two different populations, with proportions $\mathrm{p}_{1}$ and $\mathrm{p}_{2}=1-\mathrm{p}_{1}$, respectively. This would be the case whenever we can find low-conductivity inclusions in an otherwise transmissive field (silt-clay deposits in a sandy aquifer) or the opposite (sand lenses embedded in clayeysilty deposits). Within each population, $\mathrm{K}$ can be either homogeneous or heterogeneous. The initial studies on this tropic were performed by Desbarats [1987] in sand-shale aquifers and using a numerical approach. His key finding was that the effective hydraulic conductivity is a function of the shale volume fraction, the spatial covariance structure, and the dimensionality of the flow system. In the case of isotropic hydraulic conductivity his results coincide with those obtained by Dagan [1979] using a self-consistent approach.

[95] Numerical approaches to the problem can be subdivided respective to the actual goal. One approach is to find rule-of-thumb solutions for effective conductivity based on numerical results [McCarthy, 1991]. For example, Desbarats and Srivastava [1991] found that geometric averaging worked very well in their particular problem. Another possibility is to export results based on small perturbations. This was the approach taken by Rubin [1995], who provided a closed-form solution for the isotropic case,

$$
\begin{aligned}
\mathrm{K}_{\mathrm{eff}}= & \exp \left(\mathrm{m}_{1} \mathrm{p}_{1}+\mathrm{m}_{2} \mathrm{p}_{2}\right) \\
& \times\left(1+\frac{\mathrm{p}_{1} \sigma_{1}^{2}+\mathrm{p}_{2} \sigma_{2}^{2}+\mathrm{p}_{1} \mathrm{p}_{2}\left(\mathrm{~m}_{1}-\mathrm{m}_{2}\right)^{2}}{6}\right),
\end{aligned}
$$


where $\mathrm{m}_{\mathrm{i}}$ and $\sigma_{\mathrm{i}}^{2}$ are the mean and variance of the $\log$ conductivity subset $i(i=1,2)$, respectively. This result was questioned by Rubin because in a bimodal medium the global $\sigma_{\mathrm{Y}}^{2}$ value can be quite high. By means of comparison with numerical simulation performed by Desbarats [1990], Rubin [1995] found that the result obtained by a selfconsistent approach is much closer to the numerical solution than (81). Assuming no variability within the facies, i.e., $\sigma_{1}^{2}=$ $\sigma_{2}^{2}=0$, the self-consistent approach leads to the following solution

$$
\mathrm{K}_{\mathrm{eff}}=\frac{1}{3}\left(\frac{\mathrm{p}_{1}}{\mathrm{~K}_{1}+\mathrm{K}_{\mathrm{eff}}}+\frac{\mathrm{p}_{2}}{\mathrm{~K}_{2}+\mathrm{K}_{\mathrm{eff}}}\right) .
$$

[96] Analytical solutions based again on a self-consistent approach for the particular case of ellipsoidal inclusions in an otherwise homogeneous medium were presented by Pozdniakov and Tsang [2004]. They provided a suite of analytical solutions depending on the dimensionality of the problem and whether the orientation of the ellipsoids is fixed or random. Simple cases lead to closed-form solutions. As an example, for parallel flow conditions and inclusions (facies 1) elongated and highly conductive, the effective conductivity in the direction of the inclusions is given as the solution of

$$
-\widehat{\mathrm{K}}_{\text {eff }}+\varepsilon\left[-\mathrm{p}_{2} \widehat{\mathrm{K}}_{1}-\mathrm{p}_{1}\right] \sqrt{\widehat{\mathrm{K}}_{\mathrm{eff}}}+\frac{\varepsilon \widehat{\mathrm{K}}_{1}}{\sqrt{\widehat{\mathrm{K}}_{\text {eff }}}}+1+\mathrm{p}_{1}\left(\widehat{\mathrm{K}}_{1}-1\right)=0,
$$

where $\widehat{\mathrm{K}}_{\text {eff }}=\mathrm{K}_{\text {eff }} / \mathrm{K}_{2}, \widehat{\mathrm{K}}_{1}=\mathrm{K}_{1} / \mathrm{K}_{2}$, and $\varepsilon$ is the ellipsoid aspect ratio $(\leq 1)$. The value in the perpendicular direction is $\mathrm{K}_{\text {eff }}=\mathrm{K}_{2}$, provided that $\widehat{\mathrm{K}}_{1}$ is very large.

[97] Jankovic et al. [2003] used a self-consistent approach in a medium composed of a large number of spherical inclusions of different $\mathrm{K}$ values drawn from a lognormal distribution embedded in a matrix of conductivity $\mathrm{K}_{0}$. The volume of the inclusions is fixed. This field can be considered as a bimodal distribution where one of the two populations (the matrix) has a Dirac delta pdf. In this medium, $\mathrm{K}_{\mathrm{eff}}$ is obtained as the solution of the following equation:

$$
\int_{0}^{\infty} \frac{K_{\text {eff }}-K}{K_{\text {eff }}+K / 2} p(K) d K=0,
$$

which is actually equivalent to (38).

[98] Another possibility is to derive empirical rules for the $\mathrm{K}_{\text {eff }}$ values based on extension of the solution for a single inclusion and then test them numerically [Knudby, 2004]. For high permeable inclusions, Knudby arrived at a compact expression,

$$
\mathrm{K}_{\text {eff }}^{-1}=\left(\mathrm{K}_{\mathrm{A}}^{-1}-\mathrm{K}_{\mathrm{H}}^{-1}\right) \frac{\mathrm{p}_{1}-\mathrm{p}_{1}^{-1}\left(1-\mathrm{L}_{\mathrm{n}}\right)^{2}}{\mathrm{p}_{1}-\mathrm{p}_{1}^{-1}}+\mathrm{K}_{\mathrm{H}}^{-1},
$$

where $L_{n}$ is an average normalized distance between the inclusions in the direction of mean flow. Equation (85) was assumed by Knudby [2004] to be an extension of the formula presented by Bumgardner [1990] for a single inclusion.

\subsection{Media Composed by Multiple Materials}

[99] Although one can find several examples of hydrogeologic models based on the description of geological features and several approaches have emerged to deal with highly heterogeneous natural formations composed of multiple geological facies (for reviews see Koltermann and Gorelick [1996] and Winter et al. [2003, and references therein]), there are only a few applications of these concepts for determining effective or pseudoeffective properties in such media.

[100] As discussed by Winter et al. [2003], there are different conceptual models which can be used to represent media with multiple materials. Composite media models, which are typically nonstationary, can be used when a sufficient amount of data is available. In essence, a composite medium model conceptualizes the porous formation as a doubly stochastic process where randomness is due to both (1) the random geometry of blocks of different materials (or facies) and (2) the distribution of properties such as hydraulic conductivity within each facies.

[101] One group of composite media models corresponds to homogeneous media with randomly located spherical inclusions. Here the geometry of facies is unknown while properties of materials are deterministic. Early studies of effective properties in such media were carried out by Batchelor [1974]. Later Begg and coworkers [e.g., Begg and King, 1985; Begg et al., 1985] analyzed the effect of inclusions of an arbitrary shape.

[102] Shvidler [1986, 1988] treated a composite medium as a dual continuum, thus modeling the system by continuum superimposition of the different phases, and derived formal expressions for effective conductivity of a random composite [Shvidler, 1986] and a periodic medium [Shvidler, 1988]. The particular case of steady state and transient saturated flow in a confined aquifer where the hydraulic conductivity is arranged into a set of doubly periodic "black" and "white" rectangles, corresponding to two completely different materials, was studied analytically by Kacimov et al. [1999]. They calculated the effective conductivity of the medium and showed it to be an anisotropic tensor.

[103] Bertin et al. [2000] studied the case of inclusions of low permeability placed in a high-permeability matrix by means of a double-porosity model. They decompose the domain into two subdomains and show analytically that flow through the inclusions can be decomposed into two different regimes corresponding to source flow (not contributing to effective conductivity) and translation flow (contributing to $\mathrm{K}_{\mathrm{eff}}$ ). Then, temporal variations in the flow regime translate differently into the two subdomains, leading to variations in $\mathrm{K}_{\text {eff }}(\mathrm{t})$. Bertin et al. defined different relaxation times for each subdomain.

[104] Tartakovsky and Guadagnini [2004] used random domain decomposition [Winter and Tartakovsky, 2000] to 
derive and analyze the pseudoeffective conductivity of geologic media composed of several materials with uncertain geometries and conductivities. Tartakovsky and Guadagnini provided an analytical solution for $\mathrm{K}_{\mathrm{eff}}$ for the special case of one-dimensional steady state flow in a bounded domain in the absence of sources. The porous medium is composed of two materials that are separated by a (randomly located) contact point; hydraulic conductivity within each material forms a stationary random process. Upon considering the location, $\beta$, of the point of contact between the two materials as a truncated Gaussian variable with mean $\langle\beta\rangle$ and standard deviation $\sigma_{\beta}$ the pseudoeffective conductivity of the random composite medium becomes

$$
\mathrm{K}_{\mathrm{eff}}{ }^{-1}(\mathrm{x})=\frac{\operatorname{erf}(\mathrm{u})-\operatorname{erf}\left(\mathrm{u}_{0}\right)}{\operatorname{erf}\left(\mathrm{u}_{1}\right)-\operatorname{erf}\left(\mathrm{u}_{0}\right)}\left[\frac{1}{\mathrm{~K}_{\mathrm{h} 1}}-\frac{1}{\mathrm{~K}_{\mathrm{h} 2}}\right]+\frac{1}{\mathrm{~K}_{\mathrm{h} 1}},
$$

where

$$
u=\left(\frac{x-\langle\beta\rangle}{\sqrt{2} \sigma_{\beta}}\right), \quad u_{0}=-\frac{\langle\beta\rangle}{\sqrt{2} \sigma_{\beta}}, \quad u_{1}=\frac{1-\langle\beta\rangle}{\sqrt{2} \sigma_{\beta}},
$$

and $\mathrm{K}_{\mathrm{hi}}=\mathrm{K}_{\mathrm{Gi}} \exp \left(-\sigma_{\mathrm{Y}_{\mathrm{i}}}{ }^{2} / 2\right)$, where $\mathrm{i}=1,2$, are the harmonic means of $\mathrm{K}_{\mathrm{i}}(\mathrm{x})$. These expressions reveal that $\mathrm{K}_{\text {eff }}(\mathrm{x})=\mathrm{K}_{\mathrm{hi}}$, when $\mathrm{x}$ is deep within material $\mathrm{i}$, in accordance with the classical results of Dagan [1989]. If the geometry is deterministic $\left(\sigma_{\beta}=0\right), \mathrm{K}_{\text {eff }}(x)$ becomes a step function.

[105] A different approach has been undertaken by $L u$ and Zhang [2002], who described a medium composed of a multiplicity of materials by means of a multimodal spatial variability according to which a number of mutually exclusive materials (or categories) fill space. The spatial distribution of categories, each of which represents a geologic facies, has its own architecture, described by indicator random variables. Their main result is that a bimodal log conductivity field may be well approximated by an equivalent unimodal field when one of the two materials is dominant. Although Lu and Zhang did not explicitly recognize it, it can then be argued that in this case one can employ classical solutions for effective parameters in twodimensional unimodal formations. Contrariwise, when the bimodal distribution has two more or less equally important modes, it cannot be adequately represented by an equivalent unimodal distribution. We also note that Neuman [2003] has demonstrated that the multiscale model of Di Federico and Neuman [1997] and Di Federico et al. [1999] is consistent with the type of multimodal representation of $\mathrm{Lu}$ and Zhang [2002].

\section{MEAN PARALLEL FLOW: EQUIVALENT PARAMETERS}

[106] One of the questions arising in groundwater flow problems is how to treat heterogeneity in aquifer models. For this purpose it is important to find equivalent parameters, i.e., parameters capable of reproducing some average behavior for any volume of the discretized system at a certain scale. A representative scale of this volume does not necessarily need to coincide with that at which we have obtained our measurements (data support). Equivalent parameters are associated with a particular geometry and boundary conditions and are defined as spatial averages computed within a single realization of the (otherwise random) process. The problem of finding equivalent parameters is termed upscaling [Wen and Gómez-Hernández, 1996], because it involves increasing the size of the domain over which the parameters are defined. A review of upscaling methods is given by Cushman et al. [2002] and Farmer [2002]. The latter classified upscaling methods in terms of the relationship between length scales of heterogeneity and upscaling grid. Here we focus less on the methods and present only the results related to equivalent parameters, some of which were already included in the review by Renard and de Marsily [1997].

\subsection{Block Averaging}

[107] The literature in equivalent (block) parameters is quite recent. Rubin and Gómez-Hernández [1990] provided the pioneering paper on the topic of block conductivity in two-dimensional flow and square numerical blocks. They defined block conductivity (in this section we use $\mathrm{K}_{\mathrm{b}}$ or $\mathrm{K}_{\mathrm{eq}}$ interchangeably to denote equivalent hydraulic conductivity) as the ratio between the average local flow and the average gradient in a given block, both of which are computed along the direction of the expected head gradient value. The mathematical definition of $\mathbf{K}_{\mathrm{b}}$, which is assumed to have tensorial properties, is

$$
\overline{\mathbf{q}}=-\mathbf{K}_{\mathrm{b}} \overline{\nabla h} .
$$

The above equation has already been presented here as (5). The over bar indicates averaging in the spatial sense, as defined in (6).

[108] Gómez-Hernández [1991] developed a methodology to obtain all the components of the tensor in (5) for a two-dimensional case; this methodology consists of two steps: (1) computing the flow for different boundary conditions generating variations in the mean flow direction and (2) using least squares to find the optimal values of $\mathrm{K}_{\mathrm{b}, \mathrm{xx}}$, $\mathrm{K}_{\mathrm{b}, \mathrm{y} y}$, and $\mathrm{K}_{\mathrm{b}, \mathrm{xy}}$. An alternative approach is to project average flow vector and head gradient over a given direction d. By multiplying both sides of (5) by d, one can define the directional equivalent conductivity $\mathrm{K}_{\mathrm{b}}$ as the scalar value that satisfies

$$
\mathrm{K}_{\mathrm{b}}=-\frac{\int_{\mathrm{V}} \mathbf{q} \cdot \mathbf{d} \mathrm{dV}}{\int_{\mathrm{V}} \nabla \mathrm{h} \cdot \mathbf{d d V}} .
$$

[109] In general, we would have to rely on numerical solutions to obtain $\mathrm{K}_{\mathrm{b}}$. As an example, Figure 8 depicts a sketch of the method. If we were able to solve the flow problem for a very refined mesh (Figure $8 \mathrm{a}$ ), then a block could be isolated (Figure 8b) where we would know the heads and flows along the boundaries. The $\mathrm{K}_{\mathrm{b}}$ value in a given direction, let us say the $x$ direction, would be obtained 


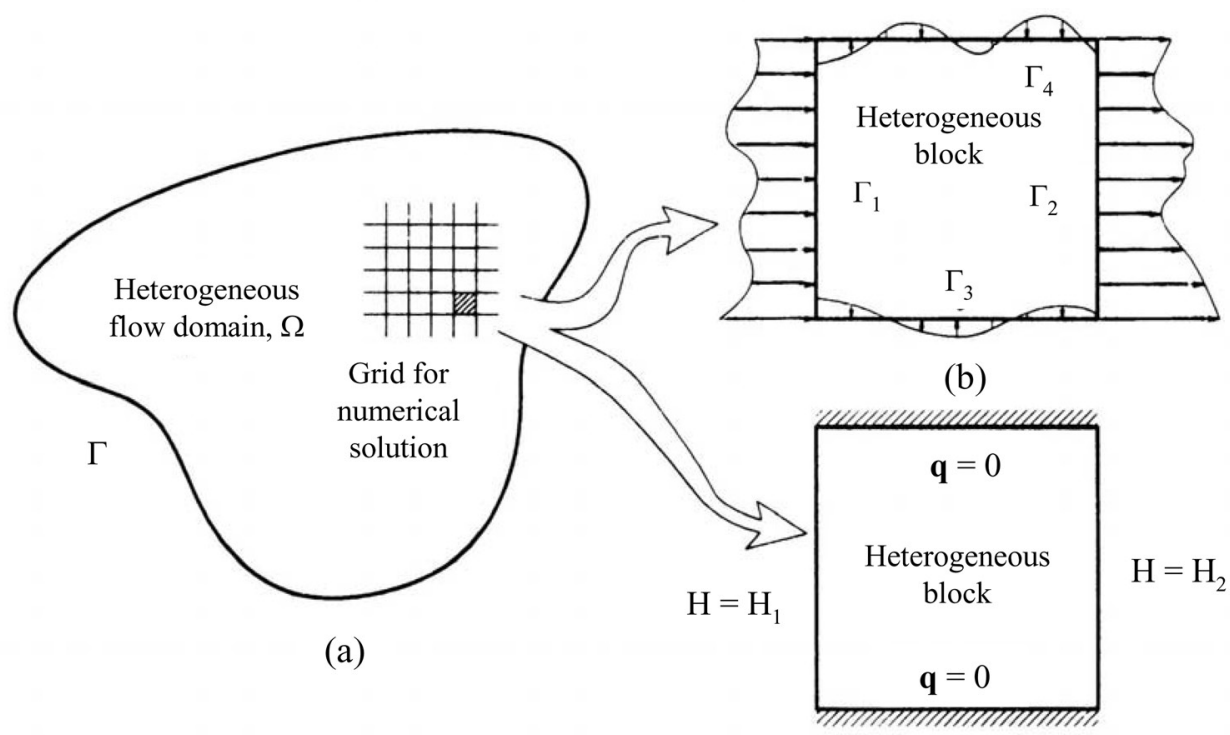

(c)

Figure 8. Derivation of block conductivities: (a) refined mesh, (b) isolated block, and (c) simple boundary conditions. The approaches of Rubin and Gómez-Hernández [1990] and of Indelman and Dagan [1993b] are based on studying the block in Figure 8b under the same conditions of the regional flow domain (Figure 8a). The Darcian approach is based on applying the boundary condition indicated in Figure 8c.

by setting $\mathbf{d}=(1,0)$ in $(88)$. The two quantities involved in (88) would then be [Sanchez-Vila et al., 1995]

$$
\begin{gathered}
\int_{\mathrm{V}} \mathbf{q} \cdot \mathbf{d} \mathrm{dV}=\frac{\mathrm{Q}_{1}+\mathrm{Q}_{2}}{2} \mathrm{~L}_{\mathrm{x}}+\frac{\mathrm{Q}_{3}-\mathrm{Q}_{4}}{2}\left(\mathrm{x}_{\mathrm{G} 3}-\mathrm{x}_{\mathrm{G} 4}\right)+\left(\mathrm{Q}_{3}+\mathrm{Q}_{4}\right) \\
\cdot\left(\frac{\mathrm{x}_{\mathrm{G} 3}+\mathrm{x}_{\mathrm{G} 4}}{2}-\frac{\mathrm{x}_{1}+\mathrm{x}_{2}}{2}\right) \\
\int_{\mathrm{V}} \nabla \mathrm{h} \cdot \mathbf{d} \mathrm{dV}=\int_{\Gamma} \mathrm{h} \mathbf{d} \cdot \mathbf{n} \mathrm{d} \Gamma-\int_{\mathrm{V}} \mathrm{h} \nabla \cdot \mathbf{d d V}=\left(\overline{\mathrm{H}}_{2}-\overline{\mathrm{H}}_{1}\right) \mathrm{L}_{\mathrm{y}},
\end{gathered}
$$

where $\mathrm{Q}_{\mathrm{i}}$ is the flow rate across the $i$ th boundary and $x_{\mathrm{Gi}}$ is the center of gravity of the fluxes at the boundary. In mildly heterogeneous aquifers, and also when the blocks are very large compared to the integral scale, the first term in the right-hand side of (89) is the dominant one. Under this situation the actual solution can be obtained by using simpler boundary conditions, such as those specified in Figure $8 \mathrm{c}$, which guarantee $\mathrm{Q}_{3}=\mathrm{Q}_{4}=0$. We stress the point that these are the boundary conditions imposed in most of the numerical studies of equivalent conductivities in the literature, regardless of this limitation.

[110] By using a perturbation approach, Rubin and Gómez-Hernández [1990] derived a general expression for $\mathrm{K}_{\mathrm{b}}(\mathbf{x})$ from (88),

$$
\mathrm{K}_{\mathrm{b}}(\mathbf{x})=\mathrm{K}_{\mathrm{G}}\left[1+\overline{\mathrm{Y}^{\prime}(\mathbf{x})}+\frac{1}{2} \overline{\mathrm{Y}^{\prime 2}(\mathbf{x})}+\frac{1}{\left\langle\mathrm{~J}_{1}\right\rangle}\left(\overline{\mathrm{Y}^{\prime}(\mathbf{x}) \mathrm{j}_{1}^{\prime}(\mathbf{x})}-\overline{\mathrm{Y}^{\prime}(\mathbf{x})} \overline{\mathrm{j}_{1}^{\prime}(\mathbf{x})}\right)\right],
$$

where $\left\langle\mathrm{J}_{1}\right\rangle$ is the ensemble mean (uniform) gradient and $\mathrm{j}^{\prime}{ }_{1}(\mathbf{x})$ is the residual of the head gradient, $\mathrm{j}_{1}$, caused by aquifer heterogeneity at a given point $\mathbf{x}$. With such a definition, $\mathrm{K}_{\mathrm{b}}(\mathbf{x})$ is an SRF, characterized by its mean, $\left\langle\mathrm{K}_{\mathrm{b}}\right\rangle$, and variance, $\sigma_{\mathrm{K}_{\mathrm{b}}}{ }^{2}$,

$$
\begin{gathered}
\left\langle\mathrm{K}_{\mathrm{b}}\right\rangle=\mathrm{K}_{\mathrm{G}}\left[1+\frac{\sigma_{\mathrm{Y}}^{2}}{2}+\frac{1}{\left\langle\mathrm{~J}_{1}\right\rangle}\left(\mathrm{C}_{\mathrm{Yj}_{1}}(0)-\overline{\mathrm{C}_{\mathrm{Yj}_{1}}}\right)\right] \\
\sigma_{\mathrm{K}_{\mathrm{b}}}^{2}=\mathrm{K}_{\mathrm{G}}^{2} \overline{\mathrm{C}_{\mathrm{Y}}},
\end{gathered}
$$

where $\mathrm{C}_{\mathrm{Yj}_{1}}\left(\mathbf{x}-\mathbf{x}^{\prime}\right)$ is the cross covariance of $\mathrm{Y}$ and $\mathrm{j}_{1}$ and

$$
\begin{aligned}
\overline{\mathrm{C}_{\mathrm{Yj}_{1}}} & =\frac{1}{\mathrm{~V}^{2}} \int_{\mathrm{V}} \int_{\mathrm{V}} \mathrm{C}_{\mathrm{Yj}_{1}}\left(\mathbf{x}-\mathbf{x}^{\prime}\right) \mathrm{d} \mathbf{x} \mathrm{d} \mathbf{x}^{\prime} \\
\overline{\mathrm{C}_{\mathrm{Y}}} & =\frac{1}{\mathrm{~V}^{2}} \int_{\mathrm{V}} \int_{\mathrm{V}} \mathrm{C}_{\mathrm{Y}}\left(\mathbf{x}-\mathbf{x}^{\prime}\right) \mathrm{d} \mathbf{x} \mathrm{d} \mathbf{x}^{\prime} .
\end{aligned}
$$

[111] Gómez-Hernández [1991] expanded this work to simulate directly block conductivity values conditioned upon measurements at the local scale. A further extension allows providing the moments of the natural logarithm of the block conductivity, $\mathrm{Y}_{\mathrm{b}}(\mathbf{x})=\ln \mathrm{K}_{\mathrm{b}}(\mathbf{x})$, as [Sanchez-Vila et al., 1995]

$$
\begin{gathered}
\mathrm{Y}_{\mathrm{b}}(\mathbf{x})=\ln \mathrm{K}_{\mathrm{G}}+\overline{\mathrm{Y}^{\prime}(\mathbf{x})}+\frac{1}{2} \overline{\mathrm{Y}^{\prime 2}(\mathbf{x})}+\frac{1}{\left\langle\mathrm{~J}_{1}\right\rangle} \\
\cdot\left(\overline{\mathrm{Y}^{\prime}(\mathbf{x}) \mathrm{j}_{1}^{\prime}(\mathbf{x})}-\overline{\mathrm{Y}^{\prime}(\mathbf{x})} \overline{\mathrm{j}_{1}^{\prime}(\mathbf{x})}\right)-\frac{1}{2}\left(\overline{\mathrm{Y}^{\prime}(\mathbf{x})}\right)^{2}, \\
\left\langle\mathrm{Y}_{\mathrm{b}}\right\rangle=\langle\mathrm{Y}\rangle+\frac{\sigma_{\mathrm{Y}}^{2}-\overline{\mathrm{C}_{\mathrm{Y}}}}{2}+\frac{1}{\left\langle\mathrm{~J}_{1}\right\rangle}\left(\mathrm{C}_{\mathrm{Yj}_{1}}(0)-\overline{\mathrm{C}_{\mathrm{Y}_{\mathrm{j}}}}\right), \\
\sigma_{\mathrm{Y}_{\mathrm{b}}}^{2}=\overline{\mathrm{C}_{\mathrm{Y}}} .
\end{gathered}
$$


[112] These analytical results can be further simplified when the correlation function of $\mathrm{Y}$ is isotropic and the blocks are either squares (in two dimensions) or cubes (in three dimensions). Under these simplifying conditions,

$$
\begin{gathered}
\left\langle\mathrm{Y}_{\mathrm{b}}\right\rangle=\langle\mathrm{Y}\rangle+\left(\frac{1}{2}-\frac{1}{\mathrm{n}}\right)(1-\alpha) \sigma_{\mathrm{Y}}^{2} \\
\sigma_{\mathrm{Y}_{\mathrm{b}}}^{2}=\alpha \sigma_{\mathrm{Y}}^{2},
\end{gathered}
$$

where $\alpha$ depends on the normalized block size (with respect to the integral distance) and the variogram model, as illustrated in Figure 9.

[113] A complete analysis of a three-dimensional block conductivity tensor was performed by Zijl and Stam [1992] for a thin grid block using a perturbations expansion. Their main result was that the zero-order term is symmetric and positive definite, with principal components equal to the arithmetic and harmonic depth averages of the local conductivities along the horizontal and vertical directions, respectively. The first-order correction (to be superimposed to this zero-order term) is, in general, a nonsymmetric tensor, and so principal directions do not exist. Stam and Zijl [1992] further provided guidelines to discern situations where higher-order terms can be neglected.

[114] Equivalent conductivity is extremely influenced by boundary conditions. Wessel-Berg and Bergmo [2002] derived analytical expressions for $\mathrm{K}_{\mathrm{b}}$ in the case of a block where part of the boundary is impervious and flow only takes place through a few gaps.

\subsection{Bounds and Combinations}

[115] The most restrictive bounds to equivalent conductivities descend from an extension of equation (8):

$$
\mathrm{K}_{\mathrm{H}} \leq \mathrm{K}_{\mathrm{eq}, \mathrm{ii}} \leq \mathrm{K}_{\mathrm{A}} \quad(\mathrm{i}=1,2,3) .
$$

This implies that the equivalent value for any direction is bounded between the harmonic and the arithmetic mean of the local $\mathrm{K}$ values within the block. The difference between this equation and (8) is that now averages are taken in the physical rather than in the probability space. These bounds are quite restrictive, and a number of authors have attempted to find less restrictive bounds. A comprehensive summary of the different bounds available in the literature is given by Renard and de Marsily [1997].

[116] A combination of the limits of (100) can be used to obtain a back-of-the-envelope calculation of $\mathrm{K}_{\mathrm{eq}}$ in a given block. The idea is based on an extension of Matheron's equation (20a) to the real space, thus writing

$$
\mathrm{K}_{\mathrm{eq}}=\mathrm{K}_{\mathrm{A}}^{\frac{\mathrm{n}-1}{\mathrm{n}}} \mathrm{K}_{\mathrm{H}}^{\frac{1}{\mathrm{n}}} .
$$

[117] While this equation is formally equal to (20a), the conceptual difference is, again, that averages are now taken within the block. On the basis of the idea of flow parallel or perpendicular to layering, Cardwell and Parsons [1945]

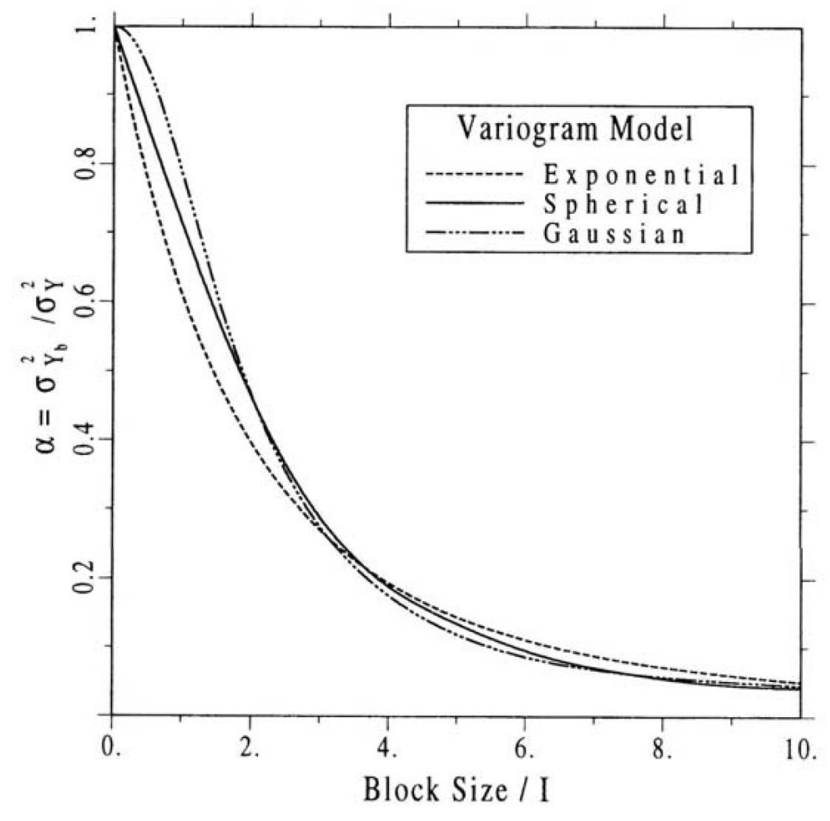

Figure 9. Parameter $\alpha$ (defined in (99)) as a function of normalized block size and correlation model [from SanchezVila et al., 1995].

derived improved bounds for $\mathrm{K}_{\mathrm{eq}}$ by progressively averaging along the three coordinate directions within a block composed by $\mathrm{N} \times \mathrm{N} \times \mathrm{N}$ elements,

$\frac{1}{\mathrm{~N}^{2}} \sum_{\mathrm{m}} \sum_{\mathrm{n}} \frac{\mathrm{N}}{\sum_{1} \frac{1}{\mathrm{~K}_{\mathrm{lmn}}}}=\mathrm{K}_{\mathrm{min}} \leq \mathrm{K}_{\mathrm{eq}} \leq \mathrm{K}_{\max }=\mathrm{N}\left[\sum_{1} \frac{\mathrm{N}^{2}}{\sum_{\mathrm{m}} \sum_{\mathrm{n}} \mathrm{K}_{\mathrm{lmn}}}\right]^{-1}$,

where $1, \mathrm{~m}$, and $\mathrm{n}$ stand for the block numbering in the $\mathrm{x}_{1}$, $\mathrm{x}_{2}$, and $\mathrm{x}_{3}$ directions, respectively. The left-hand-side member of (102) corresponds to the arithmetic average along $x_{2}$ and $x_{3}$ of the harmonic average along $x_{1}$. The righthand-side term is the harmonic average along $\mathrm{x}_{1}$ of the arithmetic averages along $\mathrm{x}_{2}$ and $\mathrm{x}_{3}$.

[118] As these bounds are narrower than those provided by (100), averaging them could be a good approximation for $\mathrm{K}_{\mathrm{eq}}$ in certain cases. This idea was suggested by Guérillot et al. [1990], who proposed using a simple combination of the $\mathrm{K}_{\min }$ and $\mathrm{K}_{\max }$ values defined in (102) for $\mathrm{K}_{\mathrm{eq}}$

$$
\mathrm{K}_{\mathrm{eq}}=\sqrt{\mathrm{K}_{\min } \cdot \mathrm{K}_{\max }} .
$$

Extensions of the Carwell-Parsons-Guérillot methodology to account for rather different bounds and combinations of these were presented by Romeu [1994].

\subsection{Power Averaging}

[119] Since the work of Journel et al. [1986] many authors have used a combined analytical-numerical method to estimate equivalent parameters based on a power-averaging formula. Gómez-Hernández and Gorelick [1989] present the discrete version of a spatial average $\mathrm{p}$ norm as 
an estimator of $\mathrm{K}_{\mathrm{b}}$ for very large blocks (large enough so that the equivalent value can also be viewed as a pseudoeffective value) according to

$$
\mathrm{K}_{\mathrm{b}}=\left[\frac{1}{\mathrm{~N}} \sum_{\mathrm{i}=1}^{\mathrm{N}} \mathrm{K}_{\mathrm{i}}^{\mathrm{p}}\right]^{1 / \mathrm{p}}
$$

[120] The immediate extension of (104) to derive block conductivity values was presented by Desbarats [1992] by defining

$$
\mathrm{K}_{\mathrm{b}}=\left[\frac{1}{\mathrm{~V}} \int_{\mathrm{V}} \mathrm{K}(\mathbf{x})^{\mathrm{p}} \mathrm{dV}\right]^{1 / \mathrm{p}}
$$

and further providing the statistics of $\mathrm{Y}_{\mathrm{b}}$ as

$$
\begin{gathered}
\left\langle\mathrm{Y}_{\mathrm{b}}\right\rangle=\ln \mathrm{K}_{\mathrm{G}}+\frac{\mathrm{p}}{2}\left(\sigma_{\mathrm{Y}}^{2}-\overline{\mathrm{C}_{\mathrm{Y}}}\right) \\
\sigma_{\mathrm{Y}_{\mathrm{b}}}^{2}=\overline{\mathrm{C}_{\mathrm{Y}}}
\end{gathered}
$$

[121] Simple average values can be derived directly from (105). When $\mathrm{p}=1,0$, and -1 , we would obtain $\mathrm{K}_{\mathrm{b}}=\mathrm{K}_{\mathrm{A}}$, $\mathrm{K}_{\mathrm{G}}$, and $\mathrm{K}_{\mathrm{H}}$, respectively. These are exact results for some particular cases (e.g., for a perfectly stratified block) but can be used as fast simple values to obtain adequate results in real examples when only a rough approximation is needed [e.g., Phillips and Belitz, 1991]. Sarris and Paleologos [2004] suggested a directional $\mathrm{p}$ value for the directional equivalent value in anisotropic fields. They found that the $\mathrm{p}_{1}$ value (in the direction of flow) that best fit their numerical results is given as a bilinear relationship in terms of the anisotropy ratio, e, and normalized block size.

[122] An important point to stress is that this type of averaging procedure relies on an a priori knowledge of the different averages. These are generally estimated from available data, and therefore estimation errors can cause relevant bias of the averaged values. An example is the work of Jensen [1991], who analyzed several alternative methods of estimating the geometric mean of the point $\mathrm{K}$ values by properly weighting the extreme values.

[123] An alternative use of power-averaged upscaled values is proposed by Desbarats and Bachu [1994]. They presented a real case where local transmissivity values obtained directly from vertical averaging over the aquifer depth, B, of local hydraulic conductivity values clearly overestimate the transmissivity values needed to fit their data. They proposed the following definition for upscaled transmissivity, $\mathrm{T}_{\mathrm{b}}$, obtained empirically:

$$
\mathrm{T}(\mathbf{x})=\mathrm{BK}_{\mathrm{B}}(\mathbf{x}) ; \mathrm{K}_{\mathrm{B}}(\mathbf{x})=\left[\frac{1}{\mathrm{~B}} \int_{0}^{\mathrm{B}} \mathrm{K}^{\mathrm{p}}(\mathbf{x}) \mathrm{dx}\right]^{1 / \mathrm{p}} .
$$

\subsection{Numerical ad hoc Methods}

[124] Fenton and Griffiths [1993] adopted a numerical Monte Carlo approach to study block conductivity statistics within a bounded rectangular domain. They concluded that the block conductivity was reasonably modeled by a lognormal distribution and that its mean and variance were closely approximated by using the statistics of local averages conductivities. Thus they confirmed numerically the validity of (98) and (99) in two dimensions.

[125] In cross-bedded sediments the core-scale anisotropic nature can influence the components of the three-dimensional $\mathbf{K}_{\mathrm{b}}$ tensor so that its off-diagonal terms cannot be neglected in general [Bierkens and Weerts, 1994; Pickup et al., 1994]. An accurate, though highly intensive, computational method for upscaling is that developed by Chen et al. [2003]. It is based on a coupled local-global upscaling approach that employs global coarse-scale simulations to determine the boundary conditions for the fine-scale evaluation of $\mathrm{K}_{\mathrm{b}}$ within an iterative process.

[126] Most of the analyses already presented deal with blocks that are considered as rectangles or parallelepipeds. An exception is offered by He et al. [2002], who introduced an approach for the calculation of the $\mathrm{K}_{\mathrm{b}}$ values in a general quadrilateral grid cell. The method consists of triangulating the cell so that the internal heterogeneity is accurately resolved and then solving flow within the block numerically.

[127] Numerical upscaling has the disadvantage of selecting a priori the shape and size of the blocks. Sometimes this results in inconsistencies at the boundaries, as blocks do not adapt properly to some particular features. This problem can by avoided by selective gridding, where the grid is not defined a priori but rather is obtained by an iterative procedure [Wen and Gómez-Hernández, 1998]. The geometry of the coarse grid is built in order to have minimum variances of flow velocities within the coarse blocks. Selective upscaling is capable of handling complex geological formations and flow patterns and provides a full equivalent hydraulic conductivity tensor for each block. These ideas were then extended by Wen et al. [2003], who presented a numerical method based on the concept of border regions (defined as a group of fine-scale cells surrounding the coarse block).

[128] Numerical approaches for obtaining equivalent conductivity values have several advantages. One of them is their possibility of handling sensitivity analysis to different conditions. As an example, Flodin et al. [2004] analyzed the impact of several different local boundary conditions (constant head, no flow, periodic, and mirror periodic) on the calculated equivalent permeability values.

[129] A general combined analytical-numerical method is that of homogenization. This method assumes that the spatial function $K(\mathbf{x})$ is periodic, with a periodicity cell, $\Omega$, which is much smaller that the aquifer size. The differential equations are then solved with periodic boundary conditions. This formulation was used by Dykaar and Kitanidis [1992] and Lough et al. [1997] to obtain upscaled values on a coarse grid. By using a homogenization method, a number of authors have provided upper and lower bounds for the principal values of $\mathrm{K}_{\mathrm{b}}$ [Rubinstein and Torquato, 1989; Trykozko and Zijl, 2002; Telega and Bielski, 2003]. A comprehensive summary of homogenization methods and 
variational principles applied to obtaining bounds for $\mathrm{K}_{\mathrm{b}}$ was given by Torquato [2002]. The errors in the homogenization upscaling associated with the boundary conditions and the size of the blocks were analyzed by $W u$ et al. [2002].

[130] Another approach is based on wavelet theory. The function $\mathrm{Y}(\mathbf{x})$ is treated as a noise signal, and wavelet theory is used to filter out the higher frequencies (denoising process), leading to a representation at the coarse scale [Panda et al., 2000]. The final result depends on the wavelet functions selected. As an example, Nilsen and Espedal [1996] used a simple piecewise bilinear function, thus producing a highly competitive algorithm, in terms of computational speed.

\subsection{Renormalization}

[131] A highly efficient numerical method for obtaining equivalent conductivities is that of renormalization [Le Loc'h, 1987; King, 1988]. The method consists of upscaling progressively, so that in each iteration a group of four elements (in two dimensions), arranged as in a $2 \times 2$ mesh, is replaced by a single upscaled value. This is done for the full domain, so that the net result is a new mesh where the elements have a double size in each direction, and the total number of elements is a quarter of the initial value. If more upscaling is needed, a new iteration of the process is performed. The main point is to find the upscaled value for the individual $2 \times 2$ (in two dimensions) or $2 \times 2 \times 2$ (in three dimensions) elements. This is generally done numerically. One should notice that in this type of configuration the selection of the numerical method is a crucial step, as finite differences and finite elements could provide quite different results. Another relevant aspect is related to the boundary conditions used in the evaluation of the upscaled value. Usually, simple Dirichlet and Neumann boundaries are used in the evaluation. Alternative boundary conditions consist of applying periodic boundary conditions to the block, forcing mean flow in two different directions, in order to obtain the full $\mathrm{K}_{\mathrm{b}}$ symmetric tensor, as suggested by Gautier and Noetinger [1997].

[132] A very good summary of the methodology of renormalization and some results were presented at about the same time by Renard and de Marsily [1997] and Gautier and Noetinger [1997]. One of the advantages of renormalization is its ability to capture high-permeability well-connected paths. Nevertheless, when upscaling is performed over very large blocks, a large degree of regularization is included in the problem, and the local details are smoothed out rapidly [Tegnander and Gimse, 1998]. Renormalization is used to directly obtain estimates of the uncertainty associated with the equivalent coarse grid [Hastings and Muggeridge, 2001].

[133] A variation of the method was presented by Renard et al. [2000]. The new method is called simplified renormalization and consists of successive grouping of meshes of size $2 \times 1(2 \times 1 \times 1$ in three dimensions $)$. Depending on their relative disposition with respect to the mean flow direction, each group of two elements is replaced by a single value that is either the arithmetic or the harmonic mean (depending on whether the two elements are placed in parallel or in series with respect to the mean flow direction). At each iteration the order of the grouping is changed. Depending on the disposition used in the first iteration, a different equivalent parameter value is obtained in a prespecified block. All possibilities are tested, and the final $\mathrm{K}_{\mathrm{b}}$ is provided as an average of the individual upscaled values.

\subsection{Energy Dissipation Methods}

[134] Indelman and Dagan [1993b, 1993c], and at about the same time Bøe [1994], developed a method for upscaling based on the concept of energy dissipation. They define $\mathbf{K}_{\mathrm{b}}$ as the tensor that maintains energy dissipation, E, within a block. Energy dissipation is defined as the rate of dissipation of mechanical energy per unit weight of fluid and is a stochastic process locally defined as

$$
\mathrm{E}(\mathbf{x})=\nabla^{\mathrm{T}} \mathrm{hK} \nabla \mathrm{h}
$$

where the superscript $\mathrm{T}$ indicates transpose. The concept of energy dissipation is very attractive, as it displays several convenient properties: first, its positive definiteness and additivity, and second, whenever energy dissipation is conserved within each block, global conservation of energy is insured. Hersvik and Espedal [1998] concluded that if and only if this condition is preserved, then the mean velocity in the full domain is also conserved.

[135] Exact upscaled values would then stem from the following equality that should be applied to each given block in the coarse discretization

$$
\nabla^{\mathrm{T}} \mathrm{hK} \nabla \mathrm{h}=\nabla^{\mathrm{T}} \widetilde{\mathrm{h}} \mathbf{K}_{\mathrm{b}} \nabla \widetilde{\mathrm{h}}
$$

Here $\widetilde{h}$ is the head computed at the coarse scale. A proper application of the method would require the solution of the fine-scale problem. Therefore it is necessary to cast the problem in statistical form and derive statistics for $\mathrm{K}_{\mathrm{b}}$. In isotropic domains with regular size blocks, Indelman and Dagan [1993a] find

$$
\begin{gathered}
\mathrm{K}_{\mathrm{b}, \mathrm{G}}=\mathrm{K}_{\mathrm{G}}\left[1+\left(\frac{1}{2}-\frac{1}{\mathrm{n}}\right) \sigma_{\mathrm{Y}}^{2}\right] /\left[1+\left(\frac{1}{2}-\frac{1}{\mathrm{n}}\right) \sigma_{\mathrm{Y}_{\mathrm{b}}}^{2}\right] \\
\sigma_{\mathrm{Y}_{\mathrm{b}}}^{2}=\frac{1}{\Omega^{2}} \int \mathrm{H}(\mathrm{r}) \mathrm{C}_{\mathrm{Y}}(\mathrm{r}) \mathrm{d} \mathbf{r}
\end{gathered}
$$

Here $\mathrm{K}_{\mathrm{b}, \mathrm{G}}$ is the geometric mean of the $\mathrm{K}_{\mathrm{b}}$ values, and $\mathrm{H}(\mathrm{r})$ is defined as the joint area of overlap of two elements of the partition $\Omega$ whose centers are located at a distance $r$. In the case of an axisymmetric partition the expression becomes [Indelman and Dagan, 1993c]

$$
\begin{gathered}
\mathrm{K}_{\mathrm{b}, \mathrm{ii}}=\tilde{\mathrm{K}}_{\mathrm{G}, \mathrm{ii}} \exp \left(\mathrm{Y}_{\mathrm{b}}^{\prime}\right) \\
\mathrm{Y}_{\mathrm{b}}^{\prime}(\mathbf{x})=\frac{1}{\Omega} \int_{\Omega} \mathrm{Y}^{\prime}\left(\mathbf{x}+\mathbf{x}^{\prime}\right) \mathrm{d} \mathbf{x}^{\prime},
\end{gathered}
$$


and $\tilde{\mathrm{K}}_{\mathrm{G}, \mathrm{ii}}$ are the upscaled hydraulic conductivity geometric means. The latter is an expression that involves $\mathrm{K}_{\mathrm{G}}$, the dimensionality of the problem, $\sigma_{\mathrm{Y}}^{2}, \sigma_{\mathrm{Y}_{\mathrm{b}}}{ }^{2}$, and a number of integrals in Fourier space. This upscaling methodology was successfully used by Bierkens [1996] to analyze a complex confining layer at various spatial scales.

\subsection{Other Methods}

[136] A simple and fast upscaling method was presented by Aasum et al. [1993]. The idea is to subdivide the block into four equally sized subblocks. In each subblock the full tensor is obtained by, first, finding the principal directions through a simple search system and then obtaining the principal values of the tensor by a combination of arithmetic and harmonic means. Then, an analytical method is used to obtain the full block tensor, based on an extension of the method presented by Kasap and Lake [1990] for two adjacent blocks.

[137] The pseudoempirical approach of Pozdniakov and Tsang [1999] is conducive to the following expressions for the statistics of $\mathrm{K}_{\mathrm{b}}$ :

$$
\begin{gathered}
\mathrm{K}_{\mathrm{b}, \mathrm{G}}=\mathrm{K}_{\mathrm{G}} \exp \left[\sigma_{\mathrm{Y}}^{2}\left(\frac{1}{2}-\mathrm{g}_{\mathrm{s}}\right)\right] \\
\sigma_{\mathrm{Y}_{\mathrm{b}}}^{2}=\varsigma_{\mathrm{s}} \sigma_{\mathrm{Y}}^{2},
\end{gathered}
$$

where $\varsigma_{\mathrm{s}}$ and $g_{\mathrm{s}}$ are upscaling functions. Pozdniakov and Tsang showed that by a proper choice of these upscaling functions it is possible to recover the results of Desbarats [1992].

[138] Another approach is the weighted output least squares (WOLS) approach [Nielsen and Tveito, 1998], which casts the issue of finding upscaled parameters in the framework of optimization problems. A norm is defined that corresponds to the distance between the velocities computed at the coarse and the fine scales. By using optimization methods they find that it is possible to write the problem in terms of minimization of a functional that only incorporates the coarse scale, so that one does not need to solve the fine scale. The applicability of this method is questionable for low-permeability blocks. Holden and Nielsen [2000] proposed an alternative WOLS method, which is based on the coupled minimization of the velocity norm and an energy norm (similar to the problem defined in section 3.5). They called this method "global upscaling." Their method needs the computation at the fine scale, so its applicability for large real problems is questionable.

[139] The analysis of transient equivalent conductivities has also been addressed through the study of continuous time random walks (CTRW). While most of the applications of CTRW to the computation of equivalent conductivity values have been carried out in fractured media, the initial application was performed in porous media [McCarthy, 1993]. Actually, the methodology allows obtaining diffusivity values, and therefore some hypotheses must be made regarding an effective storativity value. The work was then extended by Noetinger and Estebenet [2000] and complemented mathematically by Telega and Bielski [2003].

[140] Upscaling can also be carried out by means of renormalization group analysis. The methodology has already been presented in section 2 , as some of the methods included in this group also allow obtaining effective parameters. Depending on the filtering methods used to truncate the series in the spectral space, different methods are available in the literature, all of them highly demanding in numerical terms [Hristopulos and Christakos, 1999; Eberhard et al., 2004].

[141] An analytical approach based on the analysis of variance decomposition of $\mathrm{Y}(\mathbf{x})$ inside the block was proposed by Veneziano and Tabaei [2001]. Using this approach, they find an expression for $\mathrm{K}_{\mathrm{b}}$ written as

$$
\mathrm{K}_{\mathrm{b}}=\mathrm{K}_{\mathrm{G}} \exp \left[\mathrm{f}\left(\widehat{\sigma}_{\mathrm{i}}^{2}\right)\right]
$$

where $f$ stands for a linear combination of the $\widehat{\sigma}_{\mathrm{i}}^{2}$ terms, which are some of the components that are obtained from the application of the analysis of variance method.

\subsection{On the Practical Use of Equivalent Parameters}

[142] Durlofsky [1992] compared methods to define upscaled parameters in order to represent properly average flow values. He compares the performance of (1) a local upscaled method [Durlofsky, 1991], which consists of assigning block permeability values in two-dimensional formations by numerically averaging the velocity fields calculated on fine-scale grids over periodic boundary conditions; (2) a sampling method, assigning an individual sampled local value to the full block; and (3) a global upscaled value assigned to the full domain. He concludes that, in general, the local upscaled values perform best in this comparison.

[143] The conceptual and technical difficulties of upscaling have led some researchers [e.g., Cushman, 1986] to stress the need for measurement devices that could acquire data at a predefined scale in order to avoid upscaling issues. Following this idea, some work has been devoted to answer the specific question, How does a measurement device integrate the local spatial data? A general approach would be that proposed by Tidwell and Wilson [1999a], who interpreted the measured value as an already upscaled value,

$$
\mathrm{K}_{\mathrm{b}}^{\mathrm{p}}(\mathbf{x})=\int_{\mathrm{V}} \beta\left(\mathbf{x}-\mathbf{x}^{\prime}\right) \mathrm{K}^{\mathrm{p}}\left(\mathbf{x}^{\prime}\right) \mathrm{d} \mathbf{x}^{\prime}, \quad \int_{\mathrm{V}} \beta\left(\mathbf{x}^{\prime}\right) \mathrm{d} \mathbf{x}^{\prime}=1,
$$

where $\mathrm{V}$ is the volume sampled by the measuring device and $\beta$ is a weighting function that is unique to the instrument. A similar formula was proposed by Tidwell et al. [1999] (i.e., they set $p=1$ in (118)). From equation (118) it is possible to obtain an approximate implicit expression for $\mathrm{Y}_{\mathrm{b}}$

$$
\begin{aligned}
& \mathrm{Y}_{\mathrm{b}}(\mathbf{x}) \approx \int_{\mathrm{V}} \beta\left(\mathbf{x}-\mathbf{x}^{\prime}\right) \mathrm{Y}\left(\mathbf{x}^{\prime}\right) \mathrm{d} \mathbf{x}^{\prime}+\frac{\mathrm{p}}{2} \int_{\mathrm{V}} \beta\left(\mathbf{x}-\mathbf{x}^{\prime}\right) \\
& \cdot\left(\mathrm{Y}^{2}\left(\mathbf{x}^{\prime}\right)-\mathrm{Y}_{\mathrm{b}}^{2}(\mathbf{x})\right) \mathrm{d} \mathbf{x}^{\prime} .
\end{aligned}
$$


The effects of having different weighting functions for upscaling in the measurement devices have been analyzed by Zlotnik and Zurbuchen [2003].

[144] While in most cases upscaled hydraulic conductivities are used in groundwater flow and/or solute transport simulations, in some cases they are interesting by themselves. One such example is the work of Benson et al. [1994] on quality control of compacted soil liners. They performed a numerical analysis and obtained liners with an equivalent $\mathrm{K}_{\mathrm{b}}$ value associated with a high probability of being below a given threshold.

\section{CONVERGENT FLOW}

[145] Nonuniform flow in saturated porous media has been addressed in the stochastic subsurface hydrology literature much less frequently than uniform flow. This is so despite the fact that most field methods used to obtain conductivity or transmissivity values rely on convergent or divergent flow scenarios. Therefore problems associated with multidimensional nonuniform flows, of the kind encountered around extraction or injection wells, remain largely unsolved, particularly those of solute transport. A significant body of literature on representative parameters under convergent flow conditions has emerged during the last few years. We emphasize that all the results we review here start from the premise that a formation can be modeled in terms of a single geologic unit (or facies) with mild heterogeneity. As such they might not be appropriate to describe the type of structural heterogeneity discussed in sections 2.7 and 2.8, where bimodal properties and multiple materials are considered, respectively. This is a crucial point that has not yet been addressed in the stochastic literature when dealing with representative parameters of generally nonuniform flows and that might have a profound impact on the way one describes fluid and contaminant movement in a generally statistically heterogeneous unit.

\subsection{Pseudoeffective Parameters}

[146] Starting from the work of Shvidler [1962] and Matheron [1967], the search for effective medium hydraulic parameters under convergent flow conditions has been the subject of a plethora of publications. As will be demonstrated in the following, the common finding of all these works is that the constitutive equation (average Darcy's law) for nonuniform flow has a nonlocal structure and that it is generally not possible to capture all relevant aspects of convergent flow in a randomly nonuniform medium by a single effective hydraulic conductivity or transmissivity. Instead, a pseudoeffective parameter (hydraulic conductivity/ transmissivity), rendered by a convolution operator over space, can be defined in some cases. This property depends on the distance from the pumping well. In the following we will present the main analytical and numerical results by separately considering two- and three-dimensional scenarios.

\subsubsection{Two-Dimensional Analysis}

[147] Most existing work deals with two-dimensional flow, where heads and fluxes are averaged over the vertical.
A common approach has been to model the natural logarithm $\mathrm{Y}=\ln \mathrm{T}$ of aquifer transmissivity ( $\mathrm{T}$ ) as a statistically homogeneous, multivariate Gaussian random field with a given variance and spatial correlation function. There are many cases when a two-dimensional approach can be considered a proper modeling choice. First, many aquifers are of limited thickness, particularly when compared to the potentially large planar distances involved in a flow analysis. This might change when dealing with solute transport, where plume characteristic lengths are sometimes comparable with the domain extension along the third dimension. Second, in fractures the nature of flow is mainly twodimensional. Last, in most real applications, all available data regarding heads or conductivities are already obtained as vertical spatial averages (wells and piezometers are typically screened through the aquifer thickness).

[148] From the assumption that $\mathrm{T}$ is an SRF, it follows that hydraulic heads and fluxes are also random. As an immediate extension of the mean parallel flow scenario a pseudoeffective transmissivity, $T_{\text {eff }}$, is defined as the negative ratio between expected flux and head gradient at any radial distance from a well.

[149] Matheron [1967] used a Green's function approach to investigate two-dimensional flow through an annulus surrounding a well of zero radius and assumed fixed head drawdown at the well and zero drawdown at some large distance. He then provided an analysis of the first two statistical moments of specific discharge in the limiting situations when this annulus is very close or very far from the singular extraction point. His main result is that the pseudoeffective transmissivity is equal to the arithmetic mean, $T_{A}$, of the local $T$ values near the well and equal to the harmonic mean, $\mathrm{T}_{\mathrm{H}}$, at points far away from the well. These findings appear to be independent of the choice of correlation function of T. Matheron actually quotes M. I. Shvidler as the first to state that a single effective value independent of location could not be defined in such a flow configuration. The near- and far-field limits obtained by Matheron [1967] were later recovered by Naff [1991] by means of a perturbative analysis in an unbounded domain.

[150] Dagan [1989] considered a prescribed flow rate boundary condition at the well and by simple asymptotic considerations concluded that if a well in an unbounded aquifer pumps at a constant deterministic rate, then $\mathrm{T}_{\text {eff }}$ near the well is equal to $T_{H}$, while $T_{\text {eff }}$ far from the well is equal to its mean uniform flow counterpart, i.e., the geometric mean, $\mathrm{T}_{\mathrm{G}}$. Contrariwise, if hydraulic head is prescribed at the well, the pseudoeffective transmissivity at the well should become equal to $T_{A}$, thus recovering the near-well limit of Matheron [1967]. These limits have been verified by subsequent studies and were complemented by closedform solutions for the space-dependent pseudoeffective conductivity for unbounded [Indelman and Abramovich, 1994b] and bounded [Sanchez-Vila, 1997; Riva et al., 2001] domains.

[151] Ababou and Wood [1990] conjectured that the unconditional pseudoeffective conductivity for a Gaussian 


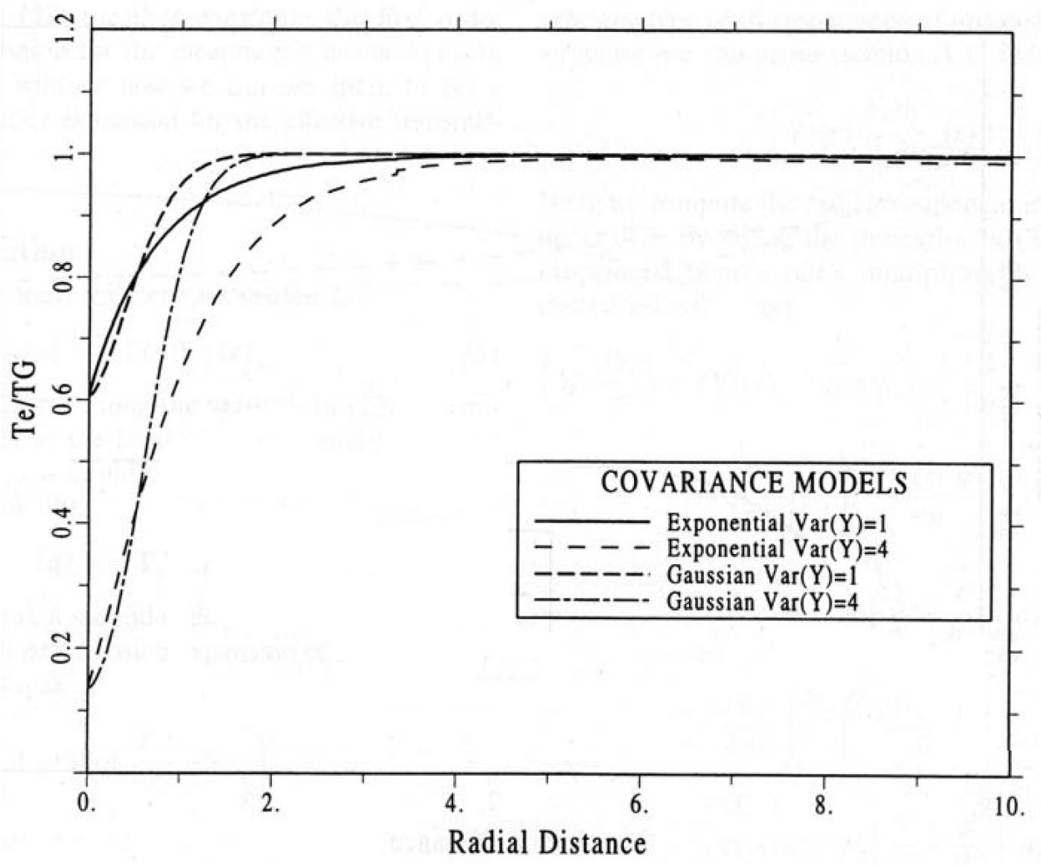

Figure 10. Dependence of normalized pseudoeffective transmissivity (122) on radial distance $\mathrm{r} / \mathrm{d}$ for the isotropic Gaussian and exponential covariance models and two different values of $\sigma_{\mathrm{Y}}^{2}\left(\sigma_{\mathrm{Y}}^{2}=1.0\right.$ and 4.0) [from Sanchez-Vila, 1997].

isotropic correlation function can be expressed as the Taylor expansion of

$$
\begin{aligned}
& \mathrm{T}_{\mathrm{eff}} \approx \mathrm{T}_{\mathrm{G}} \exp \left\{\frac{\sigma_{\mathrm{Y}}^{2}}{2}\left[\frac{\left[\ln \left(\mathrm{r}_{\mathrm{G}} / \mathrm{r}_{\mathrm{w}}\right)\right]^{2}}{\left[\ln \left(\mathrm{r}_{1} / \mathrm{r}_{\mathrm{w}}\right)\right]^{2}}-1\right]\right\} \\
& \mathrm{r}_{\mathrm{G}}=\mathrm{r}_{\mathrm{w}} \exp \left\{\sqrt{2\left[1+\left(\ln \left(\mathrm{r}_{\mathrm{w}} / \lambda\right)\right)^{2}\right]}\right\},
\end{aligned}
$$

where $r_{w}$ is the well radius, $r_{1}$ is the radius of the depression cone, and $r_{G}$ is some critical radial distance at which the pseudoeffective conductivity is equal to the geometric mean. Therefore $T_{\text {eff }}$ is larger or smaller than $T_{G}$ at distances smaller or larger than $r_{G}$, respectively. This conjecture is in contrast with later results of Indelman and Abramovich [1994a, 1994b], Indelman et al. [1996], and Riva et al. [2001], and it has the additional drawback of involving a quantity such as the radius of the depression cone, which depends on pumping duration and does not have a clear practical meaning.

[152] Shrestha and Loganathan [1994] observed that mean hydraulic heads evaluated by numerical Monte Carlo simulations were correctly interpreted using an effective medium approximation, which relies on the use of a constant effective value, equal to $\mathrm{T}_{\mathrm{H}}$. Shrestha and Loganathan did not incorporate spatial correlation in their analysis, and no details are provided on the computational grid. It can be argued that given the large flow rate and the limited radius of influence assumed by Shrestha and Loganathan, the whole domain is under the direct influence of the well, thus explaining the result that is apparently in contrast with other findings.
[153] Sanchez-Vila [1997] obtained an analytical solution for $\mathrm{T}_{\mathrm{eff}}$ in terms of perturbations and Green's functions for steady state mean radial flow within an infinite domain. He first proved that a sufficient condition for the tangential component of the expected flux to vanish $\left(\left\langle q_{\theta}\right\rangle=0\right)$ was that the correlation function of $\mathrm{Y}$ be isotropic and second-order stationary. Then, he defined $\mathrm{T}_{\text {eff }}$ in terms of the ensemble values of the radial components of flux and head gradient:

$$
\mathrm{T}_{\mathrm{eff}}=-\frac{\left\langle\mathrm{q}_{\mathrm{r}}\right\rangle}{\partial\langle\mathrm{h}\rangle / \partial \mathrm{r}}
$$

The resulting expression for $\mathrm{T}_{\text {eff }}$ to second order in $\sigma_{\mathrm{Y}}$ is

$$
\mathrm{T}_{\mathrm{eff}}=\mathrm{T}_{\mathrm{G}}\left[1+(1 / 2+\mathrm{M}) \sigma_{\mathrm{Y}}^{2}\right]
$$

where $\mathrm{M}$ is a sum of domain integrals [see Sanchez-Vila, 1997, equation (32)] of the log transmissivity correlation function and Bessel functions and varies with radial distance, r, from the pumping well. In particular, for both the exponential and the Gaussian correlation model, $\mathrm{M}$ is a monotonic increasing function of $r$, rising from $M=-1$ at the well to $\mathrm{M}=-0.5$ for infinite radial distance from the well.

[154] The actual shape of the solution can be seen in Figure 10, which shows that $\mathrm{T}_{\text {eff }}$ increases monotonically from $T_{H}$ at the well to $T_{G}$ far from the well in agreement with Dagan's [1989] observations. Indelman and Abramovich [1994b] and Sanchez-Vila [1997] noted that $T_{\text {eff }}$ reaches the asymptotic $\mathrm{T}_{\mathrm{G}}$ values at distances of about 1.5-2 integral scales from the well for the Gaussian isotropic correlation 
model and 3-5 integral scales for the exponential one. These results are consistent with numerical Monte Carlo simulations of Neuman and Orr [1993].

[155] Apart from the work of Neuman and Orr [1993] the inclusion of domain boundaries on the solution of the stochastic well problem is quite recent. Riva et al. [2001] considered the flow to a well within a randomly heterogeneous transmissivity field, where $\mathrm{Y}=\ln \mathrm{T}$ is modeled as a statistically homogeneous random field with an isotropic Gaussian correlation function. In this setting they prescribe deterministically a constant pumping rate, $\mathrm{Q}$, at the well and a constant hydraulic head at a circular outer boundary at a radius $\mathrm{L}$ from the well. Riva et al. based their analysis on the nonlocal moment equations of Guadagnini and Neuman [1999a] and developed a second-order (in $\sigma_{\mathrm{Y}}$ ) analytical solution that yields expected values of vertically averaged hydraulic head and flux and, consequently, pseudoeffective transmissivities as function of distance from the well. Their main result is encapsulated in the following expression for $\mathrm{T}_{\text {eff }}$

$$
\mathrm{T}_{\text {eff }}(\xi)=\mathrm{T}_{\mathrm{G}}\left\{1+\sigma_{\mathrm{Y}}^{2}\left[-\frac{1}{2}+\exp \left(-\frac{\pi}{4} \frac{\mathrm{L}^{2}}{\lambda^{2}} \xi^{2}\right)+\frac{\xi}{8 \pi} \frac{\mathrm{L}^{2}}{\lambda^{2}} \mathrm{I}_{\mathrm{K}}\right]\right\}^{-1}
$$

where $\xi$ is radial distance from the well, normalized to the domain radius, $\mathrm{L}$, and $\mathrm{I}_{\mathrm{K}}$ is a four-dimensional integral (given by (H15) of Riva et al. [2001]). It can be shown from (123) that $\mathrm{T}_{\text {eff }}$ tends to the harmonic mean of the local transmissivities at the well, thus recovering the limiting result presented before. This result does not depend on a predefined pdf of the log transmissivity.

[156] Figure 11 depicts the variation of $\mathrm{T}_{\text {eff }}(\xi) / \mathrm{T}_{\mathrm{G}}$ (there is a difference in notation with respect to that of Riva et al.) with normalized radial distance $\xi$ for $\sigma_{\mathrm{Y}}^{2}=0.1,1$, and 4 when $L / \lambda=5$ and 10 , respectively. Figure 11 suggests that pseudoeffective transmissivity is equal to the harmonic mean, $\mathrm{T}_{\mathrm{H}}$, at the well, tends toward the geometric mean, $\mathrm{T}_{\mathrm{G}}$, at intermediate distances, peaks above $\mathrm{T}_{\mathrm{G}}$, and then declines as one approaches the external Dirichlet boundary. At the boundary, $\mathrm{T}_{\text {eff }}$ remains somewhat larger than $\mathrm{T}_{\mathrm{G}}$. It can also be recognized that the normalized pseudoeffective transmissivity becomes approximately 1 at about $r / \lambda \approx 2$ for sufficiently large domains. From (123) it is possible to derive some limiting values for $\mathrm{T}_{\text {eff }}$ for different relative sizes of the domain:

$$
\begin{gathered}
\mathrm{T}_{\text {eff }}=\frac{\mathrm{T}_{\mathrm{G}}}{1+\sigma_{\mathrm{Y}}^{2} / 2} \cong \frac{\mathrm{T}_{\mathrm{G}}}{\exp \left[\sigma_{\mathrm{Y}}^{2} / 2\right]}=\mathrm{T}_{\mathrm{H}}, \quad \mathrm{L} / \lambda \rightarrow 0 \\
\mathrm{~T}_{\text {eff }}=\frac{\mathrm{T}_{\mathrm{G}}}{1-\sigma_{\mathrm{Y}}^{2} / 2} \cong \frac{\mathrm{T}_{\mathrm{G}}}{\exp \left[-\sigma_{\mathrm{Y}}^{2} / 2\right]}=\mathrm{T}_{\mathrm{A}}, \quad \mathrm{L} / \lambda \rightarrow \infty .
\end{gathered}
$$

[157] In a subsequent development, Neuman et al. [2004] noticed that from the theoretical and numerical results of Neuman and Orr [1993] and Riva et al. [2001], close to the well, that is in the region $\mathrm{r}_{\mathrm{w}} \leq r \leq 2 \lambda, \mathrm{T}_{\text {eff }}(\mathrm{r})$ behaves
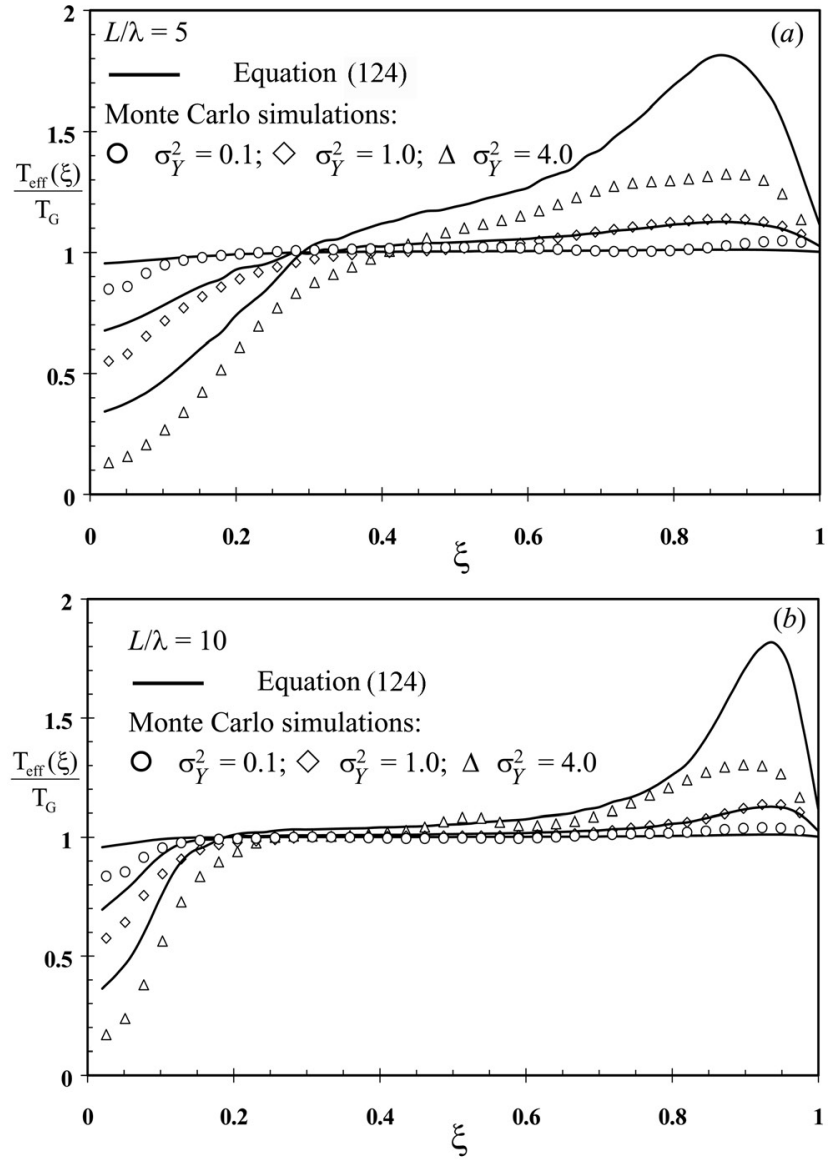

Figure 11. Normalized $\mathrm{T}_{\text {eff }}(\xi) / \mathrm{T}_{\mathrm{G}}$ versus normalized distance, $\xi$, for (a) $L / \lambda=5$ (b) $L / \lambda=10$, and various values of $\sigma_{\mathrm{Y}}^{2}$, as obtained by (123) and numerical simulations of Riva et al. [2001].

approximately as a cubic polynomial having zero derivatives at $r_{w}$ and $2 \lambda$. Then, Neuman et al. [2004] approximated $T_{\text {eff }}(r)$ in this range via

$$
\begin{gathered}
\mathrm{T}_{\text {eff }}(\mathrm{r})=\varphi_{\mathrm{H}}\left(\alpha^{\prime}\right) \mathrm{T}_{\mathrm{H}}+\varphi_{\mathrm{G}}\left(\alpha^{\prime}\right) \mathrm{T}_{\mathrm{G}}, \\
\alpha^{\prime}=\mathrm{r} /(2 \lambda), \varphi_{\mathrm{H}}\left(\alpha^{\prime}\right)=1-3 \alpha^{\prime 2}+2 \alpha^{\prime 3}, \\
\varphi_{\mathrm{G}}\left(\alpha^{\prime}\right)=3 \alpha^{\prime 2}-2 \alpha^{\prime 3} .
\end{gathered}
$$

These results can be used in the framework of geostatistical interpretation of pumping tests by means of type-curve analysis (see section 4.3).

[158] A different approach to the problem of computing $\mathrm{T}_{\text {eff }}$ in convergent flow conditions was employed by Noetinger and Gautier [1998]. They analyzed transient converging flow using a perturbations expansion combined with the diagrammatic method in order to sum up the successive terms. For a second-order expansion they were able to prove that the pseudoeffective transmissivity value obtained coincides with the steady state value for a sufficiently long test (while temporal convergence was very slow).

\subsubsection{Three-Dimensional Analysis}

[159] One of the earliest works related to the analysis of the effective hydraulic conductivity under convergent flow 
conditions was that of Warren and Price [1961], who studied the impact of a three-dimensional structure of heterogeneous and uncorrelated hydraulic conductivity on radial flow. Their key conclusion was that using the geometric mean of the lognormally distributed hydraulic conductivity provides an approximate and reasonable estimate of the behavior of the aquifer.

[160] Shvidler [1966, 1985] has subsequently developed a perturbation approach to solve a well-type flow and calculated the large time asymptotic behavior of the first-order mean head in isotropic media for distances from the source much smaller or larger than the scale of heterogeneity. He found that the inverse ratio of the first-order mean head to the homogeneous one tends to $\mathrm{K}_{\mathrm{H}} / \mathrm{K}_{\mathrm{A}}$ in the proximity of the source, while it tends to the ratio between the effective conductivity typical of uniform mean flow and $\mathrm{K}_{\mathrm{A}}$ far from the well.

[161] In statistically anisotropic heterogeneous unbounded domains, with flow driven by a constant discharge pumping well, an approximation to the pseudoeffective conductivity is provided by Naff [1991]

$$
\mathrm{K}_{\mathrm{eff}}=\mathrm{K}_{\mathrm{G}}\left[\exp \left(\sigma_{\mathrm{Y}}^{2} / 2\right)+\beta(\rho)\right],
$$

where $\rho=\mathrm{r} / \lambda$ is radial distance normalized to the horizontal (isotropic) correlation scale of log conductivity and $\beta(\rho)$ is provided at first order by equation (28) of Naff [1991]. As such, it is important to note that (128) is a mixture of firstand higher-order expressions. Naff concluded that the pseudoeffective conductivity is a first-order approximation of the arithmetic mean close to the well. It then decreases with radial distance toward a value that depends on the anisotropy ratio, e. Specifically, when e $\approx 0.01$ (horizontal correlation scale, $\lambda_{1}=\lambda_{2}$, much smaller than the vertical one, $\lambda_{3}$ ) and the flow approximates a two-dimensional scenario, the harmonic mean is nearly recovered at distances larger than $10 \lambda_{1}$. For the same distances the geometric mean would be produced when the anisotropy ratio is slightly larger than one, while the arithmetic mean would be recovered for large e values. These results are in contradiction with most of the findings available in the literature, thus questioning the validity of this mixed-order approach.

[162] From the results and approaches presented so far, it is clear that the choice of the boundary conditions (particularly the condition imposed at the well) is of the utmost importance in the analysis of flow processes around a pumping well. The relevant choices are prescribed head or prescribed flow. In three dimensions it is necessary either to state a boundary condition at any point along the vertical of the well or to use an integrated value. Thus it is not surprising that different results can be found in the literature, depending on the choice of boundary conditions used by the authors.

[163] Within this framework, early analyses of steady state flow toward a fully penetrating well were performed using a prescribed head at the wellbore. Indelman et al. [1996] used the methodology developed by Indelman and Abramovich [1994b] to model this problem as a singular line with influx proportional to the conductivity for the case of thick formations (vertical length much larger than log conductivity integral scale) and for small well radius compared to the horizontal integral scale of log conductivity. Indelman et al. [1996] derived second-order expressions for a pseudoeffective hydraulic conductivity defined as that of a fictitious homogeneous medium that conveys the same ensemble mean discharge as the heterogeneous formation for the prescribed head at the well and a given vertically averaged head at a piezometer located at a certain radial distance from the well. This definition relies on the assumption that hydraulic head is ergodic along the vertical. They arrived at the following expression

$$
\mathrm{K}_{\mathrm{eff}}=\mathrm{K}_{\mathrm{A}}\left[1-\lambda\left(\mathrm{r}, \mathrm{r}_{\mathrm{w}}, \mathrm{e}\right)\right]+\mathrm{K}_{\mathrm{efu}} \lambda\left(\mathrm{r}, \mathrm{r}_{\mathrm{w}}, \mathrm{e}\right),
$$

where $\mathrm{K}_{\text {efu }}$ is the effective conductivity for mean uniform flow in the horizontal direction in the same aquifer and $\lambda$ is a weighting coefficient that depends on location, anisotropy ratio, and the well radius. A similar approach was used by Dagan [2001], who obtained a similar result for a pseudoeffective conductivity defined in accordance with (121). On the basis of (129) it can be seen that $\mathrm{K}_{\text {eff }}$ approaches the arithmetic mean at the source (as opposed to the harmonic mean in the case of prescribed flow rate at the well). At large distances, $\mathrm{K}_{\text {eff }}$ approaches the value typical of uniform flow, as obtained previously by Indelman and Abramovich [1994b] and Sanchez-Vila [1997]. Moreover, $\mathrm{K}_{\text {eff }}$ is sensitive to the anisotropy ratio and tends slowly to its asymptotic value.

[164] Indelman [2001, 2003a] further investigated the problem of averaging transient flows driven by sources with a prescribed head boundary condition, derived an average flow equation, and defined an effective conductivity tensor for an arbitrary heterogeneous formation in the special case of uniformly distributed initial head. The relevant point of his analysis is the derivation of a quite general expression for the pseudoeffective conductivity tensor in the Fourier-Laplace space that is completely determined by the medium structure (as was also shown by Tartakovsky and Neuman [1998a] for generally nonuniform flow conditions) and accounts for either given flux or given head boundary condition at the well. Indelman then particularizes this expression for the pseudoeffective conductivity tensor in the case of an isotropic three-dimensional medium.

[165] The other group of relevant results is based on prescribing discharge at the well. Indelman [2000] analyzed the average mean head distribution for flow toward a source of instantaneous and constant discharge in unbounded randomly heterogeneous two- and three-dimensional media. He first applied perturbation and derived analytical expressions for the mean Green's function, which is the fundamental solution of the average flow equation. This allows him to show that the mean head distribution coincides asymptotically with that resulting from the application of an effective medium flow model at large distances from the instantaneous and constant rate source. These results are 


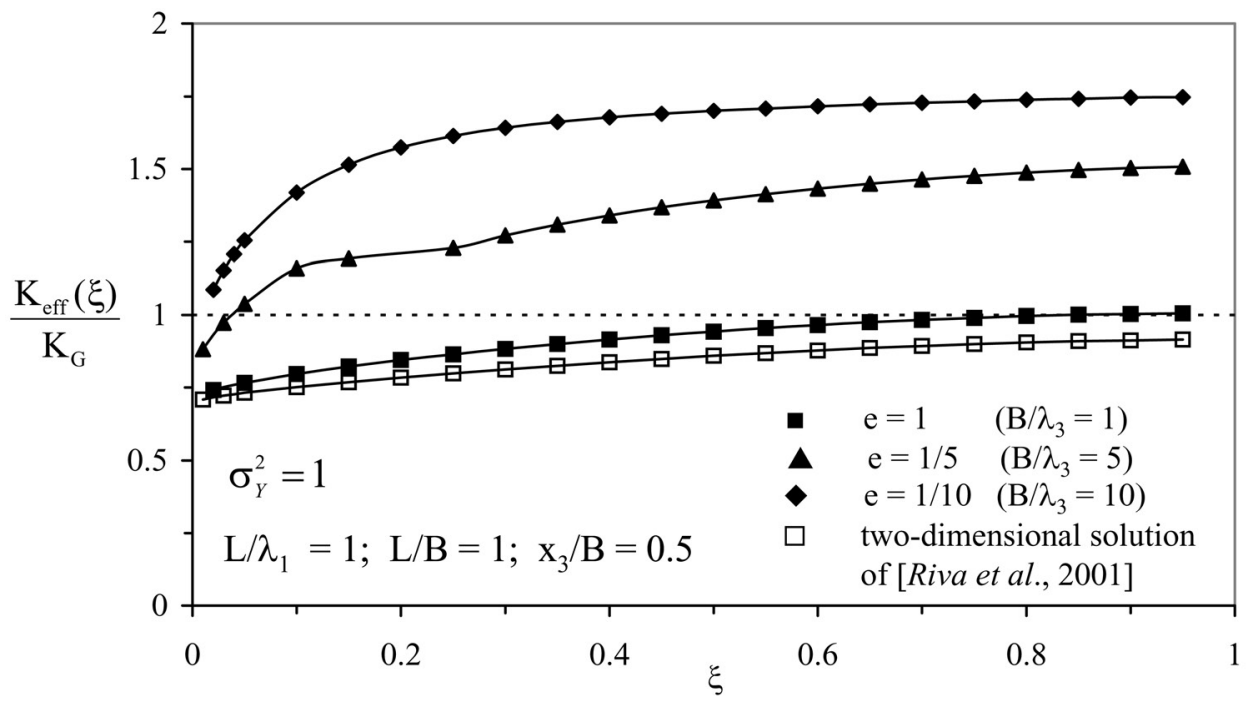

Figure 12. Normalized pseudoeffective conductivity versus normalized distance, $\xi$, for various values of the anisotropy ratio, e, at $\mathrm{x}_{3} / \mathrm{B}=0.5$ when $\mathrm{L} / \mathrm{B}=\mathrm{L} / \lambda_{1}=1 ; \sigma_{\mathrm{Y}}^{2}=1$. Here $\mathrm{L}$ is the radial distance to the external constant head boundary, $\mathrm{B}$ is aquifer thickness, $\sigma_{\mathrm{Y}}^{2}$ is variance of (natural) $\log$ conductivity. Adapted from Guadagnini et al. [2003].

then used by Indelman [2001] within the context of convergent well flow driven by a point source in an infinite medium to show that the pseudoeffective conductivity is equal to the harmonic mean at the source and then increases with distance. The value typical of uniform mean flow is reached within 2-3 integral scales from the source.

[166] The effects of a finite boundary were analyzed by Guadagnini et al. [2003]. They provide an analytical solution for pseudoeffective conductivity in a convergent flow due to a fully penetrating well, discharging at a given rate within a randomly heterogeneous, statistically anisotropic aquifer delimited by two horizontal no-flow boundaries (separated by a vertical distance, B) and a vertical boundary of cylindrical shape along which head is maintained at a prescribed value. They used two not exactly equal (though quite similar) definitions of pseudoeffective conductivity depending on whether it is defined from the ensemble average extension of Darcy's law or Thiem's solution. In the first case they used the second-order approximation of the mean flux, while in the second they used the specified total flow rate. In their work the logarithm of hydraulic conductivity forms a statistically homogeneous random field characterized by an anisotropic Gaussian spatial covariance function. Their key results are synthesized in Figures 12 and 13, illustrating the effect of statistical anisotropy ratio, e, on the variation of the nor-

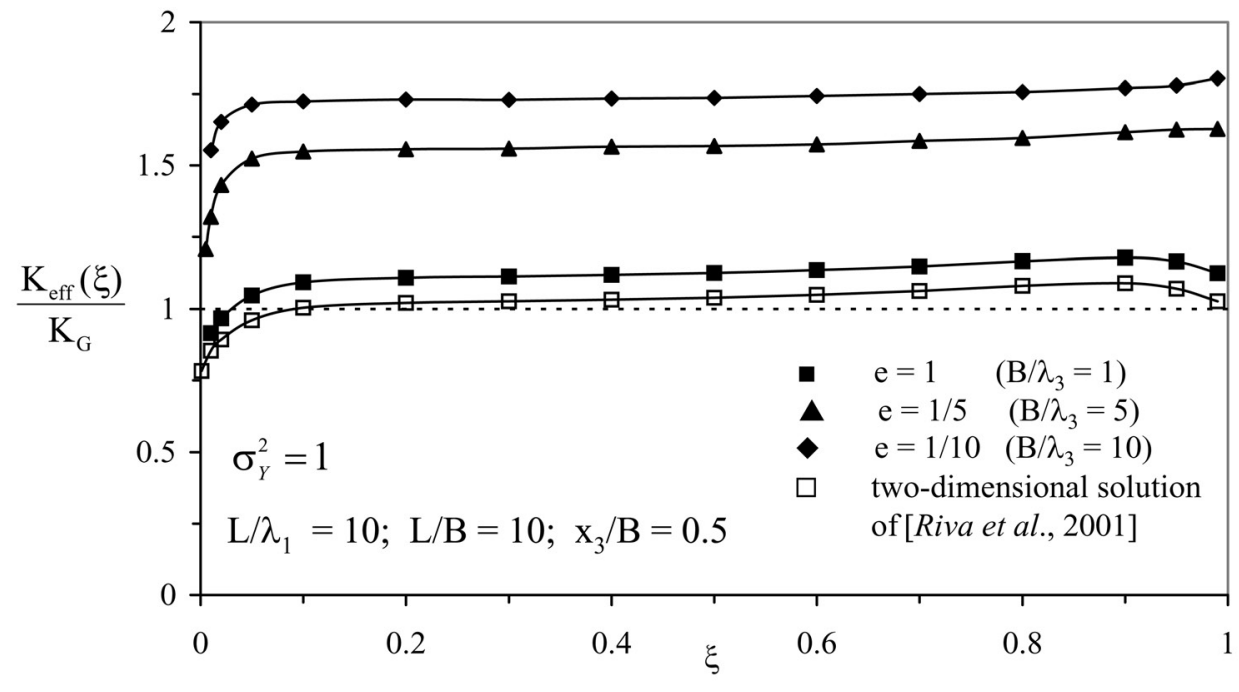

Figure 13. Normalized pseudoeffective conductivity versus normalized distance, $\xi$, for various values of the anisotropy ratio, e, at $\mathrm{x}_{3} / \mathrm{B}=0.5$ when $\mathrm{L} / \mathrm{B}=\mathrm{L} / \lambda_{1}=10 ; \sigma_{\mathrm{Y}}^{2}=1$. Here $\mathrm{L}$ is radial distance to the external constant head boundary, and B aquifer thickness. Adapted from Guadagnini et al. [2003]. 
malized pseudoeffective conductivity with dimensionless radial distance $\xi=r / L$ midway between the horizontal noflow boundaries (at $\mathrm{x}_{3} / \mathrm{B}=0.5$ ) when $L / B=L / \lambda_{1}=1$ and 10 , respectively. One of the main conclusions is that in all cases, rendering the aquifer more stratified (by decreasing e from 1 to 0.1 ) causes the pseudoeffective hydraulic conductivities to increase. This is because resistance to horizontal flow becomes weaker and the aquifer is able to deliver a prescribed discharge rate to the well under a reduced mean hydraulic gradient.

[167] The pseudoeffective hydraulic conductivity values obtained from an average extension of Thiem's formula are always larger than those obtained from an average extension of Darcy's law, though the two do not differ significantly from each other. At distances that exceed two horizontal correlation scales from the well and the outer boundaries, both definitions stabilize.

[168] Effective conductivities under steady state conditions can also be seen as the limiting values for transient effective values at large times. This was the approach used by Indelman [2003b], who derived closed-form (moderate and) large-time approximations of the mean hydraulic head in three-dimensional transient random flow driven by a fully penetrating well pumping at a constant rate. On the basis of these approximations he then offered a generalized form of the Cooper and Jacob [1946] solution by deriving a largetime asymptotic for the expected value of the drawdown. The solution reveals that the pseudoeffective transmissivity derived from the late time behavior of Indelman's solution coincides with the effective conductivity for uniform horizontal flow (see his equation (30)). Another result of interest that can be extracted from Indelman's work is related to the asymptotic expression for the first-order correction of the mean drawdown, which includes a slightly different format for the weighting function $\lambda$ of (129), which simplifies the way of calculating it. This result asymptotically recovers the steady state behavior of Indelman et al. [1996], which was derived by direct averaging of the steady state flow. Raghavan [2004, p. 13] commented on the significance of these results by stressing that if one was "to use the pressure-time curve to estimate permeability with time, then the permeability decreases with distance." This allows us to qualitatively (albeit not quantitatively) recover the behavior suggested by the analysis of the time derivative of hydraulic head observed in a number of field tests [see, e.g., Raghavan, 2004, Figure 4].

\subsection{Equivalent Parameters}

[169] As defined in section 1, the concept of an equivalent hydraulic conductivity/transmissivity is closely related to that of block support values. The underlying idea is to find a single value (scalar or tensor) that could replace a heterogeneous distribution within a domain (block) in some given sense. Under convergent flow conditions this usually means that an area surrounding the extracted well is selected, some boundary conditions are imposed, and the total outflow is measured. The equivalent conductivity would then be defined as the single value that should be assigned to the area to obtain the same total outflow observed in the heterogeneous scenario under the same boundary conditions. In this sense, equivalent parameters can be viewed as global quantities that characterize the ability of the medium to convey fluid, and they are of direct use in numerical computations of flow, when some upscaling is required. Like the pseudoeffective parameters these are generally influenced by the type of boundary conditions. While early analyses of these topics tend to offer empirical solutions, modern works try to propose analytical expressions, mainly based on moment equations and perturbation approaches.

[170] To illustrate the concept and its practical application, we consider the simple setting corresponding to an annular domain where the inner annulus is the perimeter of a pumping well. We specify prescribed deterministic heads, $\mathrm{h}_{\mathrm{w}}$ and $\mathrm{h}_{\mathrm{e}}$, at the inner and outer boundaries, respectively. These boundaries are located at radial distances $r_{w}$ and $r_{e}$ from the center of the domain. For a homogeneous medium and under steady state conditions, $\mathrm{T}$ is related the total flow rate, Q, by

$$
\mathrm{T}=\frac{\mathrm{Q}}{2 \pi\left(\mathrm{h}_{\mathrm{e}}-\mathrm{h}_{\mathrm{w}}\right)} \ln \frac{\mathrm{r}_{\mathrm{e}}}{\mathrm{r}_{\mathrm{w}}} .
$$

Equation (130) is usually known as Thiem's formula [Thiem, 1906], even though it was initially presented by Dupuit [1863]. Equation (130) is strictly valid only in confined aquifers, although it can also be used in phreatic aquifers under the restriction of small drawdown. Obviously, if the medium was not homogeneous, but rather the transmissivity displayed some variations in space, one could still apply the same boundary conditions and, for a computed or observed outflow, compute a value of block or equivalent transmissivity, $\mathrm{T}_{\mathrm{eq}}$, which is then rigorously defined as

$$
\mathrm{T}_{\mathrm{eq}}=\frac{\mathrm{Q}}{2 \pi\left(\mathrm{h}_{\mathrm{e}}-\mathrm{h}_{\mathrm{w}}\right)} \ln \frac{\mathrm{r}_{\mathrm{e}}}{\mathrm{r}_{\mathrm{w}}} .
$$

On the basis of this definition, Cardwell and Parsons [1945] proved that in a heterogeneous aquifer, $T_{\text {eq }}$ is bounded by the weighted harmonic and the weighted arithmetic averages of $\mathrm{T}(\mathbf{x})$ over the whole domain, that is,

$$
\mathrm{W}\left(\int_{\mathrm{V}} \frac{\mathrm{dV}}{\mathrm{r}^{2} \mathrm{~T}(\mathbf{x})}\right)^{-1} \leq \mathrm{T}_{\mathrm{eq}} \leq \frac{1}{\mathrm{~W}} \int_{\mathrm{V}} \frac{\mathrm{T}(\mathbf{x})}{\mathrm{r}^{2}} \mathrm{dV}, \quad \mathrm{W}=\int_{\mathrm{V}} \frac{\mathrm{dV}}{\mathrm{r}^{2}(\mathbf{x})} .
$$

With reference to this particular geometry, only a few simplistic cases of heterogeneity are amenable to producing an exact formula for $T_{e q}$. One is the case where the transmissivity values are only a function of the radial distance from the well (e.g., when they are distributed within circular, radially symmetric annuli). This configuration yields

$$
\mathrm{T}_{\mathrm{eq}}=\ln \left(\mathrm{r}_{\mathrm{e}} / \mathrm{r}_{\mathrm{w}}\right)\left(\int_{\mathrm{r}_{\mathrm{w}}}^{\mathrm{r}_{\mathrm{e}}} \frac{\mathrm{dr}}{\mathrm{rT}(\mathrm{r})}\right)^{-1}
$$


which coincides with the lower bound in (132). In contrast, when $\mathrm{T}$ is constant within sectors extending from the inner to the outer radius,

$$
\mathrm{T}_{\mathrm{eq}}=\frac{1}{2 \pi} \int_{0}^{2 \pi} \mathrm{T}(\theta) \mathrm{d} \theta
$$

which corresponds to the arithmetic mean.

[171] The first paper that we are aware of dealing with the topic of equivalent conductivities with nonuniform mean flow is that of Gómez-Hernández and Gorelick [1989]. They first discretized the domain into elements and then assigned the block $\mathrm{T}$ values by means of a discrete version of a power-averaging formulation

$$
\mathrm{T}_{\mathrm{eq}}=\left(\frac{1}{\mathrm{~V}} \int_{\mathrm{V}} \mathrm{T}(\mathbf{x})^{\mathrm{p}} \mathrm{dV}\right)^{1 / \mathrm{p}}
$$

The authors then propose the values to be assigned to the power $p$ by means of an empirical approach. In cases where the degree of heterogeneity is mild (low variance of the conductivity or $\log$ conductivity), the simple averaging methods compete favorably with more sophisticated methods as shown by numerical simulations performed by Vidstrand [2001].

[172] An alternative to upscaling is to use an individual T value drawn at random from those found within the block. This simplified approach provides reasonable results for mean uniform flow conditions but not in the case of flows driven by source terms (such as convergent flow), as shown numerically by Durlofsky [1992].

[173] Perhaps the most relevant empirical approach is that of Desbarats [1992], who employed an analogy with the mean uniform flow case and proposes an expression for $\mathrm{T}_{\text {eq }}$, which would apply in two-dimensional domains of different geometries:

$$
\mathrm{T}_{\mathrm{eq}}=\exp \left(\frac{1}{\mathrm{~W}} \int_{\mathrm{V}} \frac{\mathrm{Y}(\mathbf{x})}{\mathrm{r}^{2}} \mathrm{dV}\right), \quad \mathrm{W}=2 \pi \ln \mathrm{C} \frac{\mathrm{r}_{\mathrm{e}}}{\mathrm{r}_{\mathrm{W}}}
$$

where $\mathrm{C}$ is a shape factor which is equal to $1.0000,0.9560$, and 0.7938 for circular, square, and rectangular (aspect ratio 2:1) field geometries, respectively, and $\mathrm{r}_{\mathrm{e}}=\sqrt{A / \pi}$, A being the domain area. In his paper, Desbarats [1992] found equation (136) to work very well for multivariate logGaussian square $\mathrm{T}$ fields in which the transmissivity at the well is not very different from the expected value of T. This was somehow confirmed by Gómez-Hernández et al. [1995] by means of numerical simulations performed in a synthetically generated heterogeneous aquifer.

[174] Moreover, an upscaled parameter provided by the weighted average of the local Y values proportional to $1 / \mathrm{r}^{2}$ was found by Beckie and Harvey [2002] to provide quite good results in the context of the interpretation of slug tests in heterogeneous media.
[175] Desbarats [1994] then extended the work to three dimensions. In such a case, and again on the basis of empirical arguments, he proposed the following equation for $\mathrm{K}_{\mathrm{eq}}$ :

$$
\mathrm{K}_{\mathrm{eq}}^{\mathrm{p}}=\frac{1}{\mathrm{~W}} \int_{\mathrm{V}} \frac{\mathrm{K}(\mathbf{x})^{\mathrm{p}}}{\mathrm{r}^{2}} \mathrm{dV}
$$

Notice that (137) converges to (136) for $p=0$. From (137) it is possible to compute mean and variance of $\mathrm{K}_{\mathrm{eq}}$ [Desbarats, 1993]

$$
\begin{gathered}
\left\langle\mathrm{K}_{\mathrm{eq}}\right\rangle=\exp \left(\langle\mathrm{Y}\rangle+\frac{\mathrm{p}}{2}\left(\sigma_{\mathrm{Y}}^{2}-\bar{\sigma}\right)+\frac{\bar{\sigma}}{2}\right) \\
\operatorname{Var}\left(\mathrm{K}_{\mathrm{eq}}\right)=\left\langle\mathrm{K}_{\mathrm{eq}}\right\rangle^{2}(\exp (\bar{\sigma})-1),
\end{gathered}
$$

where $\bar{\sigma}$ is the weighted average of the covariance function of $\mathrm{Y}$, given by

$$
\bar{\sigma}=\frac{1}{\mathrm{~W}^{2}} \int_{\mathrm{V}} \int_{\mathrm{V}} \frac{\mathrm{C}_{\mathrm{Y}}\left(\mathbf{x}, \mathbf{x}^{\prime}\right)}{\mathrm{r}^{2} \mathrm{r}^{\prime 2}} \mathrm{dVdV}^{\prime}
$$

[176] Skin effects are then incorporated in this empirical methodology by Durlofsky [2000]. He considers that the bulk of the domain is well characterized by a certain $\mathrm{K}_{\text {eq }}$, which would be that corresponding to uniform in the mean flow (e.g., the geometric mean in an infinite two-dimensional domain). The effects of the heterogeneity on the nearwell flow are captured through an effective skin $\mathrm{K}$ value, obtained through (136) using a domain that is the one affected by skin effects.

[177] An approximated, perturbation-based, analytical solution to the problem of upscaling transmissivity under convergent flow conditions was performed by Sanchez-Vila et al. [1999a]. The authors found that expansion of (136) to zero and first order provided the correct solution. Instead, the expansion to second order turns out not to be correct. The complete expression of Sanchez-Vila et al. [1999a] reads

$$
\begin{aligned}
\mathrm{T}_{\mathrm{eq}}= & \mathrm{T}_{\mathrm{w}}\left\{1+\frac{1}{\mathrm{~W}} \int_{\mathrm{V}} \frac{\mathrm{Y}^{\prime}(\mathbf{x})}{\mathrm{r}^{2}} \mathrm{dV}-\frac{1}{2 \mathrm{~W}} \int_{\mathrm{V}} \frac{\mathrm{Y}^{\prime 2}(\mathbf{x})}{\mathrm{r}^{2}} \mathrm{dV}\right. \\
& +\frac{1}{\mathrm{~W}^{2}}\left[\int_{\mathrm{V}} \frac{\mathrm{Y}^{\prime}(\mathbf{x})}{\mathrm{r}^{2}} \mathrm{dV}\right]^{2} \\
& \left.+\frac{1}{\mathrm{~W}} \sum_{\mathrm{n}=1}^{\infty} \int_{\mathrm{V}} \int_{\mathrm{V}} \frac{\mathrm{Y}^{\prime}(\mathbf{x})}{\mathrm{r}^{2}} \frac{\mathrm{Y}^{\prime}\left(\mathbf{x}^{\prime}\right)}{\mathrm{r}^{\prime 2}} \mathrm{H}_{\mathrm{n}}\left(\mathbf{x}, \mathbf{x}^{\prime}\right) \mathrm{dVdV}^{\prime}\right\},
\end{aligned}
$$

where in this formula $\mathrm{Y}^{\prime}=\mathrm{Y}-\mathrm{Y}_{\mathrm{w}}, \mathrm{Y}_{\mathrm{w}}=\ln \mathrm{T}_{\mathrm{w}}$, and $\mathrm{T}_{\mathrm{w}}$ is transmissivity at the well. $\mathrm{H}_{\mathrm{n}}$ is provided by Sanchez-Vila et al. [1999a, equation (31)].

\subsection{Interpreted Parameters}

[178] A traditional way to obtain hydraulic conductivity values in field applications is by means of hydraulic testing. 
When a pump test is performed in a real aquifer, some drawdown curve is obtained. Then a method of interpretation is used to obtain a value of hydraulic conductivity (actually in most methods the value obtained is a transmissivity). The resulting parameter is somehow an integrated value of the conductivities around the pumping and observation wells (which could coincide or not). In some sense it is an equivalent value, but we chose to term it "interpreted hydraulic conductivity" (short for parameters estimated from test interpretations), as the value would not only depend on the hydraulic conductivity spatial distribution plus the boundary conditions but also on the interpretation method used. A recent review of the procedures adopted to evaluate pumping test or pressure-response curves in heterogeneous porous media was offered by Raghavan [2004]. Here we are mainly concerned with the review of results about the application of different types of hydraulic tests that are conducive to direct evaluation of aquifer effective (or pseudoeffective) parameters.

[179] Early works are those of Vandenberg [1977] and Barker and Herbert [1982], which were concerned with the interpretation of pumping tests performed within randomly heterogeneous aquifers. The single, mildly heterogeneous realization analyzed by Vandenberg [1977] did not allow discerning clearly the impact of heterogeneity. The work of Barker and Herbert [1982] was concerned with a highconductivity inclusion embedded in a regional aquifer, and the main conclusion was that the effect of the inclusion was negligible in determining the regional transmissivity, provided that there was sufficient time for the cone of depression to expand beyond the inclusion itself.

[180] Most of the analytical work has been focused on units composed of two bounded regions of different (fixed and deterministic) $\mathrm{T}$ values, separated by some sharp discontinuity (inclusions). Butler [1988] analyzed the case of a fully penetrating well (in two dimensions) at the center of a circular disk, embedded in an infinite matrix of different transmissivity. In his work the interpreted transmissivity, $\mathrm{T}_{\text {int }}$, is obtained through the method of Cooper and Jacob [1946]. The method consists of plotting the drawdowns, $s$, versus time, $t$, in a semilogarithmic plot. If the curve displays a straight line with slope $\mathrm{m}$, a value for $\mathrm{T}_{\mathrm{int}}$ is obtained from

$$
\mathrm{T}_{\mathrm{int}}=0.183 \frac{\mathrm{Q}}{\mathrm{m}}
$$

[181] Butler found that the contribution of the inner disk is negligible when analyzing the slope of the curve for very large times. Later, Butler and Liu [1993] obtained the same result for a disk arbitrarily located with respect to the pumping well. Heterogeneities at the local scale, close to the well, appear as variations in the slope of the semilogarithmic s versus $t$ plot for very short times. This effect was discussed in detail by Schad and Teutsch [1994]. Tiedeman et al. [1995] extended the work of Butler and Liu [1993] to elliptical (rather than circular) inclusions. Their work is steady state; therefore they actually used Thiem's formula instead of Cooper-Jacob's formula. By taking the shape of the ellipse to the limit, Tiedeman et al. [1995] analyzed the case of a vertical fracture of finite extension.

[182] A different method of obtaining interpreted transmissivity is based on Theis' curve-matching procedure [Theis, 1935]. Butler [1990] was the first to prove numerically that while Cooper-Jacob's method provides information about some average hydraulic conductivity at a global (large) scale, Theis' method preferably averaged the local values around the extraction point. This result was also pointed out by Serrano [1997].

[183] Some attempts have been made to derive analytical solutions for interpreted transmissivities in heterogeneous media. The first one was that of Oliver [1990], who noticed how the slope in the s versus t semilog plot changed with time and analyzed the relative contribution of the individual $\mathrm{T}(\mathbf{x})$ values to the apparent transmissivity for any given time. He found $T_{\text {int }}$ to be a weighted average of the transmissivity within a region that is a moving annular domain.

[184] Interpretation of long-time pump tests in heterogeneous media has been the subject of intensive work since the late 1990s. Meier et al. [1998] recalled a number of real cases where the interpretation of long-time pump tests led to similar values of interpreted transmissivities, while rendering very different values of interpreted storativity. Their suite of numerical simulations has led to the following key results: (1) For a given test the interpreted transmissivities derived from several observation points coincide; (2) this value was found to be equal to the equivalent parameter (independently evaluated) for all the cases analyzed; and (3) the interpreted storativity value provided a general idea about how well connected the pumping and the observation points were.

[185] The first and third conclusions have been corroborated by Sanchez-Vila et al. [1999b], who provided a perturbation-based analytical random solution for the convergent flow taking place within a single realization of a heterogeneous two-dimensional aquifer. Their analyses lead to the following second-order (in $\mathrm{Y}^{\prime}$ ) expression for $\mathrm{T}_{\text {int }}$ :

$$
\begin{aligned}
\mathrm{T}_{\mathrm{int}}= & \mathrm{T}_{\mathrm{G}} \exp \left(\frac{1}{4 \pi} \lim _{\rho \rightarrow \infty} \frac{1}{\pi \rho^{2}} \int_{0}^{\infty} \int_{0}^{2 \pi} \int_{0}^{2 \pi} \mathrm{Y}^{\prime}(\boldsymbol{\rho}) \mathrm{Y}^{\prime}\left(\boldsymbol{\rho}^{\prime}\right)\right. \\
& \left.\cdot \frac{\left(\rho^{2}+\rho^{\prime 2}\right) \cos \left(\phi-\phi^{\prime}\right)-2 \rho \rho^{\prime}}{\left[\rho^{2}+\rho^{\prime 2}+2 \rho \rho^{\prime} \cos \left(\phi-\phi^{\prime}\right)\right]^{2}} \rho^{3} \mathrm{~d} \phi \mathrm{d} \phi^{\prime} \mathrm{d} \rho^{\prime}\right)
\end{aligned}
$$

One of the key points in (143) is that $\mathrm{T}_{\mathrm{int}}$ is independent of the location, and thus the same value would eventually develop at all observation points. The interpreted storativity, $\mathrm{S}_{\text {int }}$, which is obtained from the intercept of the line developed in the Cooper-Jacob plot is provided by the following first-order (in $\mathrm{Y}^{\prime}$ ) expression:

$$
\ln \mathrm{S}_{\mathrm{int}}=\ln \mathrm{S}+\frac{1}{\pi} \int_{\mathrm{V}} \mathrm{Y}^{\prime}(\rho, \phi) \frac{\rho-\mathrm{r} \cos (\theta-\phi)}{\rho^{2}+\mathrm{r}^{2}-2 \rho \mathrm{r} \cos (\theta-\phi)} \frac{1}{\rho} \mathrm{dV}
$$


which is location-dependent. In (144), $\mathrm{S}$ is the actual storativity value, which is assumed to be constant throughout the domain. This is a self-evident case where the values of interpreted parameters are basically driven by the interpretation method selected.

[186] Copty and Findikakis [2004] challenged the second conclusion of Meier et al. [1998]. They stated that for a multi-Gaussian field the expected value of the interpreted transmissivity is not the geometric mean of $\mathrm{T}$ (as would be concluded from Meier et al.'s work) but is rather a smaller value depending on the amount of variability. Copty and Findikakis as well as Neuman et al. [2004] developed a method to obtain information about different statistical parameters characterizing the heterogeneity of the domain from individual tests. In particular, the work of Neuman et al. [2004] is conducive to the determination of the actual values of the integral distance, $\mathrm{I}_{\mathrm{Y}}$, the variance of $\mathrm{Y}$, and $\mathrm{T}_{\mathrm{G}}$, thus allowing obtaining (pseudo)effective parameters by means of the different equations presented in section 4.2.

[187] Interpreted transmissivity values can also be obtained from other types of hydraulic tests. One of them is the recovery test, where residual drawdown is recorded after ceasing pumping. The classical interpretation method is to plot residual drawdown versus equivalent time in a semilog plot. Equivalent time is defined as 1 plus the ratio between the duration of pumping and elapsed time since pumping has ended (assuming pumping rate was constant). From the slope of this plot and by means of (142) a value of interpreted transmissivity is obtained. Willmann et al. (On the meaning of the transmissivity values obtained from recovery tests, submitted to Hydrogeology Journal, 2006) found that in most cases two different slopes can be drawn from the plot, with a transition between them. From the slope developed for early times, we can obtain information about the local values around the well. The late time slope provides information about some regional value which resembles the equivalent value for mean uniform flow conditions.

[188] The last hydraulic test we will address is that of specific capacity, which consists of fixing a pumping time and computing the drawdown at the end of the period. Specific capacity is defined as the ratio between the pumping rate, Q (assumed constant), and the drawdown at the well at the end of the period, $\mathrm{s}_{\mathrm{w}}$. Interpreted transmissivity is obtained by a very simple expression,

$$
\mathrm{T}_{\text {int }}=\mathrm{A} \frac{\mathrm{Q}}{\mathrm{s}_{\mathrm{w}}},
$$

where $\mathrm{A}$ is a constant value which depends on the radius of the well.

[189] Interesting results were presented by Meier et al. [1999] in the context of a numerical study on interpreted transmissivity values derived in heterogeneous synthetic aquifers from specific capacities plotted versus (1) transmissivity values calculated from a Cooper-Jacob approach (using (142)) and (2) $\mathrm{T}$ values at the well. Their main conclusion is that the values obtained from (145) are highly dominated by the value of transmissivity at the well value. The plots relating transmissivity to specific capacity data compiled by the authors by using (142) and (145) display a convex deviation from linearity. This can be explained on the grounds that tests performed in high-conductivity areas quickly attain their large time behavior, thus tending to rapid stabilization. The opposite holds for tests carried out in low-conductivity areas. The main conclusion that can be extracted from this work is that using the geometric mean of the $T_{\text {int }}$ values obtained from short pump tests would lead to an underestimation of the effective $T$ value.

\subsection{Scale Effects}

[190] When discussing the problem of scales in section 1, we mentioned that changing scales requires answering a few questions: First, is the formal structure of the flow equation maintained after a change in the scale? Second, what are the conditions for the existence of and, in case they do exist, the values of the parameters to be used at each scale? Third, how can one relate these values at different scales? The first question has not been completely analyzed in the stochastic framework. This does not mean that it is not relevant or that there is not room for it. We conjecture that the large-scale flow equation may display delayed yield effects even if water storage is released instantaneously upon a head change. The fact that this question has not been addressed so far reflects that research has concentrated on hydraulic conductivity upscaling rather than the relevance of this issue.

[191] Therefore, when speaking of scale effects in flow processes, practitioners mean the apparent increase in representative hydraulic conductivity in response to an increase in the scale of observation. This increase has been observed in all kinds of geologic media [Neuman, 1994] but most markedly in fractured media [Brace, 1984; Bradbury and Muldoon, 1990; Clauser, 1992; Schulze-Makuch et al., 1999; Tidwell and Wilson, 2000; Meier et al., 1998; Carrera et al., 1990; Martinez-Landa and Carrera, 2005]. It must be clear that these observations stem from model interpretations or hydraulic tests. Therefore they always refer to interpreted hydraulic conductivities or transmissivities.

[192] Because of its empirical nature the cause and even the existence of scale effects in hydraulic conductivity are controversial. Many alternative explanations have been provided in an attempt to justify the field observations. Some authors attribute scale effects to factors such as a skin effect around the borehole or incomplete well development [Butler and Healey, 1998; Rovey, 1998; Rovey and Niemann, 2001] or turbulence [Lee and Lee, 1999]. These would lead to low interpreted local transmissivity values, consistently smaller than the average properties. However, well losses only affect the estimate of hydraulic conductivity in short-range tests (such as pulse and injection tests), where transmissivity is derived from total drawdown. Longrange tests, which are interpreted using the rate of drawdown change, are not sensitive to well losses because these are filtered out during interpretation. As a result they would tend to yield transmissivity values larger than short-range 
tests. Other authors attribute the scale effect to the method applied for interpreting the tests [Zlotnik and Zurbuchen, 2003]. Meier et al. [1999] attributed scale effects to the fact that interpreted transmissivities derived from pumping tests reach their equivalent value faster in high-transmissive zones than in low-transmissive zones. As a result, for a given test duration, more transmissivity estimates will be below than above the average. Guimerà et al [1995], on the other hand, attributed scale effects to the fact that long-term hydraulic tests tend to be performed in high-permeability zones. Biased sampling may be even more acute in loose material, where unaltered core samples are hard to obtain from the most permeable gravel zones.

[193] Despite the above reservations the evidence for scale effects is overwhelming. It has been observed on data collected at a variety of sites and under diverse fluid flow regimes [Carrera et al., 1990; Schad and Teutsch, 1994; Samper-Calvete and García-Vera, 1998; Rovey and Cherkauer, 1995]. Illman and Neuman [2001, 2003] and Illman [2004] interpreted single-hole and cross-hole tests performed in tuff by means of type curves and concluded that the conductivity scale effect is real. The same conclusion was obtained by Vesselinov et al. [2001], who interpreted the same tests using three-dimensional numerical inversion, while treating the medium as a stochastic, randomly heterogeneous continuum. Carrera et al. [1990] found the transmissivity of a fracture in gneiss to be 20 times larger than the geometric average of point estimates of transmissivity obtained in the fracture. Bredehoeft et al. [1983] found the effective hydraulic conductivity of the Pierre Shale, derived from regional mass balance, to be 1000 times larger than that derived from core tests. Martinez-Landa and Carrera [2005] found the interpreted hydraulic conductivity of a granite block to increase by almost 2 orders of magnitude from local values $(10 \mathrm{~cm}$ scale) derived by pulse tests to large-scale values (about $100 \mathrm{~m}$ ) derived by model calibration of cross-hole tests. Despite of all these site-specific evidences the most compelling argument is probably that of Clauser [1992]. He collected data from different sites and arranged them by scale. The resulting data increased by several orders of magnitude from the laboratory to the regional scales.

[194] The fact that scale effects are found under such a wide set of testing conditions and flow regimes suggests that the effect is real and cannot be solely attributed to conditions around the well. The practitioner's view is that increasing the scale leads to increasing the chances of encountering highly permeable regions. This type of argument is behind much of the work of Neuman and $\mathrm{Di}$ Federico and coworkers [Neuman and Di Federico, 2003, and references therein]. They argued that increasing the scale causes an increase of the log conductivity variance, so that the effective (actually pseudoeffective) hydraulic conductivity also increases.

[195] Another line of thought consists of attributing scale effects to the high connectivity of conductive zones. Sanchez-Vila et al. [1996] found that the large-scale equivalent value of transmissivity can be much larger than the geometric average, whenever points with a hightransmissivity value are better connected than points with low-transmissivity value. These findings are entirely consistent with the philosophy and results of Di Federico and Neuman [1998], Illman [2004], and Martinez-Landa and Carrera [2005]. In all these cases the full variability was sampled by the small-scale tests. High large-scale hydraulic conductivity was a result of the way the high-conductivity zones were arranged. In fact, Martinez-Landa and Carrera [2005] were able to reproduce the large-scale behavior using local values but assigning the high local conductivity values to fractures. Under this view a large-scale conductivity emerges from the medium structure. Since only a few high-conductivity zones carry most of the water, only a few local tests sample them. Yet they control the large-scale behavior of the system. Since connectivity appears to control large-scale conductivity, it is natural to try and define it, which was attempted by Knudby and Carrera [2005]. These views of scale effects bear important implications for the way the medium ought to be treated, a subject we will revisit in the concluding section of this paper.

\section{OTHER NONUNIFORM FLOW CONDITIONS}

[196] When the (average) flow pattern is not simple, the relevant question is, "Is it possible to describe a complex flow configuration by means of a single, effective, equivalent parameter"? Even though from sections $2-4$ it should be clear by now that this is generally not possible, we note that the problem has attracted some attention in the literature, starting from the late 1970s.

[197] Smith and Freeze [1979] evaluated, by means of numerical Monte Carlo simulations, the effective conductivity for a nonuniform two-dimensional flow within a rectangular domain. For their particular flow configuration they calculated an effective conductivity that was about 10 to 20 percent larger than the geometric mean. An attempt to explain this finding was proposed by Gelhar [1993], who argued that this situation of nonuniform hydraulic gradient was analogous to a scenario with a trend in the mean hydraulic conductivity, but did not provide further insight.

[198] A mild form of flow nonuniformity, consisting in a two-dimensional isotropic aquifer in the presence of a uniformly distributed recharge was then considered by Dagan [1981] in the framework of the self-consistent approach. The author concluded that the effective conductivity could properly be rendered by the geometric mean of the local conductivity values without providing a quantitative indication of the limitations of this result.

[199] A relatively complex unconfined aquifer was analyzed by Gómez-Hernández and Gorelick [1989], who simulated by means of Monte Carlo analysis the effects of the spatial variability of a variety of parameters, including hydraulic conductivity, riverbed leakance, and recharge, on the mean behavior of the system. Their key findings are that (1) the use of the geometric mean as an effective conductivity appears to be appropriate to represent mean flow far away from pumping wells and (2) contrariwise, it was not 
satisfactory near the well locations, where the equivalent conductivity was found to lie between the geometric and the harmonic mean. While we note that the first observation is consistent with findings reported in the previous sections, it is worthwhile mentioning that in the cases investigated the extent of the area around a pumping well where (ensemble) mean heads deviate from those predicted by using the geometric mean of the conductivity as a representative parameter is practically insensitive to the correlation scale. These results, as acknowledged by the authors, might have been influenced by the local flow patterns established around the modeled pumping wells.

[200] The flow regime which develops between an injection and a pumping well has been addressed by Desbarats [1993] using an empirical approach based on numerical simulations. He analyzes the concept of interwell equivalent (note that Desbarats refers to it as an effective parameter) transmissivity, defined as the transmissivity of a fictitious homogeneous medium in which one observes the same hydraulic head difference between the two wells in the doublet as in the heterogeneous aquifer. By combining the power-averaging model of Desbarats [1992] and a geostatistical model describing the local heterogeneity of transmissivity he proposes conditional and unconditional expressions for the moments of interwell transmissivity. In the unconditional case, he found that the expected value of the interwell equivalent transmissivity is bounded by the geometric and the arithmetic mean and that the lower bound is attained for interwell distances larger than ten times the correlation scale of the transmissivity. The same flow configuration was later addressed by Durlofsky [2000] with a similar approach.

[201] Another type of nonuniform flow which is relevant for practical applications is that produced by a minipermeameter. Tidwell and Wilson [1999a, 1999b] reported permeability upscaling experiments on blocks of volcanic tuff and Berea Sandstone. They compare their measurements with existing theoretical models of conductivity upscaling and found that, even though the general behavior of their data is consistent with volume averaging techniques, the nonuniform flow induced by the minipermeameter induces a nonlinear behavior of the volume averaging process, which is not predicted by available theories. It is our feeling that the development of either volume or stochastic averaging techniques including this flow configuration should be a main concern to further explore issues associated with the interpretation of minipermeameter measures.

[202] We close this section by recalling that one of the most important categories of generally nonuniform in the mean flows is that arising from conditioning of predictions (and measures of associated uncertainty) on information. Moment equations-based methods have been used to numerically analyze the nature of pseudoeffective properties in randomly heterogeneous aquifers, in the presence of hydraulic conductivity and/or hydraulic head data. In particular, Neuman and Guadagnini [2000] investigated the conceptual meaning of the parameters which are obtained by the standard practice of calibrating a deterministic flow model against (otherwise randomly) varying data. Starting from a localized version of the nonlocal moment equation of steady state flow of Guadagnini and Neuman [1999a] the mean (conditional) flow predictor can be expressed in the familiar looking Darcian form

$$
\langle\mathbf{q}(\mathbf{x})\rangle_{\mathrm{c}} \approx-\mathbf{K}_{\mathrm{c}}(\mathbf{x}) \nabla\langle\mathrm{h}(\mathbf{x})\rangle_{\mathrm{c}} \quad \mathbf{K}_{\mathrm{c}}(\mathbf{x})=\langle\mathrm{K}(\mathbf{x})\rangle_{\mathrm{c}} \mathbf{I}+\kappa_{\mathrm{c}}(\mathbf{x}) .
$$

Here the subscript $\mathrm{c}$ indicates conditioning on hydraulic conductivity and/or head data, $\boldsymbol{\kappa}_{\mathrm{c}}(\mathbf{x})$ is the conditional counterpart of (34), and $\mathbf{K}_{\mathrm{c}}(\mathbf{x})$ is the spatially varying conditional pseudoeffective hydraulic conductivity tensor. The most intriguing contribution given by Neuman and Guadagnini is that $\mathbf{K}_{\mathrm{c}}(\mathbf{x})$ constitutes a biased estimate of the actual hydraulic conductivity, $\mathrm{K}(\mathbf{x})$. While the latter is represented more faithfully by its unbiased conditional mean value, $\langle\mathrm{K}(\mathbf{x})\rangle_{\mathrm{c}}$, it is $\mathbf{K}_{\mathrm{c}}(\mathbf{x})$ rather than $\langle\mathrm{K}(\mathbf{x})\rangle_{\mathrm{c}}$ that renders (more or less) unbiased predictions of head and flux, provided that the assumptions of local uniformity of mean gradient and residual flux are approximately satisfied. The key conclusions that can be extracted from the work of Neuman and Guadagnini are that (1) whereas the hydraulic conductivity in traditional deterministic flow models was considered to be a unique material property, in reality it is a nonunique, data-dependent, spatially varying pseudoeffective quantity; and (2) calibrating a traditional deterministic flow model against measured values of head and flux is tantamount to conditioning it on such measurements. Thus pseudoeffective conductivity can be in some sense embedded in an inverse model along the lines of Rubin and Dagan [1987a, 1987b] or Hernandez et al. [2003].

\section{SYNTHESIS, CONCLUSIONS, AND ADDED VIEW}

[203] Heterogeneity is the singular most salient feature in hydrogeology. Addressing heterogeneity has been the focus of much of the most exciting and intense research of the last 30 years. The work has been so extensive that it can hardly be summarized, despite our somewhat lengthy review. Therefore this concluding section is devoted to revisiting the motivation, nomenclature, relevance, and main findings, ending with some considerations about the future.

[204] The ultimate motivation of all the work discussed here is to find representative values of hydraulic conductivity and transmissivity. The problem is posed, in ideal terms, as that of finding the value of hydraulic conductivity that ought to be used given that one knows the statistical properties of the point values of conductivity/transmissivity. This statement is ambiguous in several senses but most particularly in what is meant by "representative."

\subsection{On the Concepts of Effective, Equivalent, and Interpreted Hydraulic Conductivity}

[205] A number of definitions have been used to select the appropriate representative value. In an attempt to harmonize the different existing notation, we have classified them as effective, equivalent, and interpreted values. Effective hydraulic conductivity is the one that relates the expected 
value of flux and head gradient. Here expectation is meant as averaging in the probability space. The resulting effective value can be either a global property (i.e., constant) or a local one (i.e., varying in space). In the latter case we adopted the term pseudoeffective to account for spatial variability of the representative parameter. Equivalent hydraulic conductivity is the one that relates the spatial averages of flux and head gradient. That is, the concept of equivalent parameter refers to a single specific aquifer. Finally, interpreted parameters are those derived from interpretation of field data, typically hydraulic tests.

[206] All three definitions are relevant. The effective conductivity is, mathematically speaking, the easiest to find, and thus a large number of analytical results are available on this topic (see sections 2 and 4). The equivalent conductivity is the one that represents a specific (portion of the) aquifer and is therefore the one we would be interested in finding (see sections 3 and 4). This last statement might lead to the wrong conclusion that effective parameters lack practical interest. However, on the grounds of ergodic behavior of the system (ensemble averages equal to spatial averages) one hopes both definitions to be identical (for very large blocks). Finally, interpreted parameters are the ones that are obtained in field applications by direct measurements. Therefore it is important to find how these three representative quantities are interrelated.

[207] Our review poses several distressing questions regarding effective parameters. First, they do not always exist. When they do, a number of literature results are based on the multi-Gaussianity assumption, which has never been proven. In fact, scale effects and geological observations suggest that in actual aquifers high-conductivity zones tend to display an enhanced connection with respect to average or low-conductivity zones. Variability in connectedness is inconsistent with the multi-Gaussianity assumption. Moreover, location-independent effective parameters only exist under relatively strict conditions (infinite domain, uniform in the mean flow, etc.). Despite of these limitations, effective parameters remain the reference values for representative hydraulic conductivity and transmissivity.

[208] Equivalent parameters are not much better off. Results are often presented in terms of perturbation expansions, including truncation, without any explicit statement about the spatial distribution of the individual local conductivity values. However, actual evaluations rely on the assumption that one aquifer is an individual realization of a spatial random function, which is assumed to be fully known. Unfortunately, statistical characterization is extremely difficult. For one thing, point values of hydraulic conductivity are rarely, if ever, available. Cores are expensive and often provide biased results. Even if exact measurements of hydraulic conductivity were available, the multivariate distribution is impossible to characterize.

[209] The previous paragraphs are not as negative as they sound. They simply imply that the purported objective of effective and equivalent parameters cannot be met in practice. That is, existing approaches are not appropriate to find "representative" values of hydraulic conductivity in real aquifers. Instead, they must be considered as a framework for analyzing flow through heterogeneous media and for properly interpreting field data. It is from this perspective that the work of the last 30 years plus must be viewed.

\subsection{Results Related to Effective Parameters}

[210] 1. Regarding methods, perturbation techniques often combined with Green's function approaches remain the most widely used approach.

[211] 2. From this perspective most methods are identical up to second order in $\sigma_{\mathrm{Y}}$. That is, if $\sigma_{\mathrm{Y}}$ is small (say, significantly smaller than 1), any averaging will do.

[212] 3. The reference values of effective conductivity up to second order remain those of Gutjahr et al. [1978]: $\mathrm{K}_{\mathrm{eff}}=$ $\mathrm{K}_{\mathrm{G}}\left[1+\left(\frac{1}{2}-\frac{1}{n}\right) \sigma_{\mathrm{Y}}^{2}\right]$, which are strictly valid only for mean uniform flow in infinite aquifers.

[213] 4. Results are available for mean uniform flow in the mean flow up to sixth order in $\sigma_{\mathrm{Y}}$. The resulting expression is approximately consistent with $\mathrm{K}_{\mathrm{eff}}=\mathrm{K}_{\mathrm{G}}$ $\exp \left[\left(\frac{1}{2}-\frac{1}{n}\right) \sigma_{\mathrm{Y}}^{2}\right]$ in isotropic media.

[214] 5. Anisotropy in $\mathrm{K}_{\text {eff }}$ emerges as a natural consequence of anisotropic spatial correlation even when $\mathrm{K}$ is locally isotropic. The ratio of anisotropy in directional $\mathrm{K}_{\mathrm{eff}}$ values is a function of the variance of $(\log )$ conductivity and the correlation scales anisotropy ratio.

[215] 6. Results are also available for nonstationary log conductivity fields or bounded domains. However, in these cases an effective $\mathrm{K}$ does not exist. Instead, pseudoeffective (i.e., spatially variable) $\mathrm{K}$ must be defined. Pseudoeffective parameters must also be sought when the flow field is nonuniform. This can be the case when the expected value of $\mathrm{K}$ is variable in space or when flow is conditioned to local conductivity values.

[216] 7. Convergent flow is the most interesting case of nonuniform flow because it is the one created by a pumping well. Here the pseudoeffective $\mathrm{K}$ depends on radial distance from the well and on the boundary conditions. If flow is prescribed at the well, the $\mathrm{K}_{\mathrm{eff}}$ at the well is the harmonic mean of the hydraulic conductivity distribution, $\mathrm{K}_{\mathrm{H}}$, and grows to the value corresponding to mean uniform flow conditions (e.g., the geometric mean, $\mathrm{K}_{\mathrm{G}}$, in two dimensions) at large distances.

[217] 8. A particularly appealing approach is the residual flux method of Neuman and coworkers. The relevance of the method comes from the fact that, first, pseudoeffective parameters can be derived under general flow conditions and, second, it yields not only (pseudo)effective conductivities but also an effective equation for the moments of the dependent variables. Unfortunately, the resulting equation is difficult to work with in analytical terms. Still, in simple cases it yields the same results as other methods, and in difficult cases (bounded domains and conditioned log conductivity fields) it is amenable to numerical solution.

\subsection{Results Related to Equivalent Parameters}

[218] 1. Whenever ergodicity is valid or reasonable to invoke (e.g., uniform in the mean flow and large domain), 
equivalent $\mathrm{K}$ is identical to effective $\mathrm{K}$. In fact, the two terms can be used interchangeably.

[219] 2. However, the concept of equivalent parameters is better suited for solving the problem of upscaling. That is, given the distribution of conductivity over a fine grid, find the value that should be used over larger blocks.

[220] 3. A number of formulae are available for upscaling provided that one solves the flow equation over the fine grid. Since this is precisely what upscaling tries to avoid, the practical use of these equations is limited. However, they make it apparent that the equivalent $\mathrm{K}$ value of a block is not solely a function of the values of conductivity within such block. That is, equivalent $\mathrm{K}$ is nonlocal.

[221] 4. Nonlocality means that equivalent $K$ must be expressed as a spatial convolution spanning both point values of conductivity within the block and surrounding the block.

[222] 5. Nonlocality reflects that equivalent $\mathrm{K}$ depends on the actual boundary conditions applied to the block, which, in turn, depend on the hydraulic conductivity values surrounding the block. Still, quite good values can be obtained using simplified boundary conditions, notably using periodic boundary conditions.

[223] 6. The formal similarity between nonlocal equivalent $\mathrm{K}$ values and residual flux theory suggests that a strong link exists between these two concepts. However, this link remains to be explored.

[224] 7. Equivalent hydraulic conductivity can be viewed as a spatial random function. Its mean value and covariance function can be characterized, so that it can be conditioned to local measurements.

[225] 8. Equivalent $\mathrm{K}$, when existing, can have tensorial properties, not only because the covariance of the point conductivity values is anisotropic or because point values are tensorial but also because of the geometry of the block. In this case, equivalent $\mathrm{K}$ will tend to be smallest in the direction along which the block is largest. Equations are available for obtaining the equivalent hydraulic conductivity tensor using simplified methods such as power averaging or renormalization techniques. This result highlights the need to treat block equivalent $\mathrm{K}$ as a spatial random function. For example, working with a stack of elongated blocks with (mean) anisotropic equivalent $\mathrm{K}$ would be wrong if one is not willing to acknowledge in the model the ensemble of (variable) values of $\mathrm{K}$ in each block. This can be very complex.

[226] 9. The above may lead one to question the actual need for upscaling. One reason is related to the increasing power of available computational resources. This dramatically reduces the need for coarse-grid parameters obtained from direct numerical upscaling methods. At a more fundamental level it is recognized that stochastic averages are valid at the same scale as the original equations being averaged. This is naturally embedded in approaches based on nonlocal conditional stochastic moment equations, which provide equivalent $\mathrm{K}$ values that vary smoothly in space. This reduces dramatically the need for upscaling and space averaging.

\subsection{Results Related to Interpreted $K$ and Scale Effects}

[227] 1 . Most results related to interpreted $\mathrm{K}$ are restricted to two-dimensional scenarios because most hydraulic tests are interpreted assuming two-dimensional flow. That is, it is usual to work with transmissivity rather than hydraulic conductivity. Still, significant work has been performed recognizing that, most often, flow is indeed three-dimensional.

[228] 2. Hydraulic test interpretations that are based on total drawdown (such as variations of the Thiem equation, pulse tests, early time Theis curves, or specific capacity) yield interpreted $\mathrm{T}$ values which characterize the distribution of the transmissivity values around the well.

[229] 3. Hydraulic test interpretations that are based on the rate of growth of drawdown (such as Cooper-Jacob or recovery test interpretations) yield large-scale equivalent $T$ values. The size of the region characterized by the test depends on the duration of pumping.

[230] 4. These findings might appear to close the circle by showing that these latter interpretations indeed lead to the kind of equivalent parameters that are needed for building models. However, it is emerging that these parameters are often larger than what existing formulae for effective and equivalent parameters imply. That is, transmissivity values derived form long-time pumping tests are larger, often much larger, than the geometric average of point transmissivity values predicted by theory.

[231] 5. An important point is the existing relationship between the ensemble values of interpreted parameters in order to allow direct comparison with the actual statistics of the spatial random function (assumed known). Thus much information could be derived from geostatistical interpretations of hydraulic tests. This topic is still in a very preliminary stage.

[232] 6. The topic of scale effects in conductivity and transmissivity is not settled. In fact, some authors attribute these effects to the flow geometry around the well. Yet we feel that the body of evidence is large enough to consider these effects real and frequent. We attribute them to high connectivity of high conductivity (or transmissivity) values.

\subsection{In Conclusion}

[233] The number of results related to representative hydraulic conductivities is impressive. The last 30 years have indeed been fruitful. Still, a number of challenges remain. These include the following: (1) the need for further insights on when and under what conditions pseudoeffective, equivalent, and interpreted parameters are interchangeable; (2) an analysis of the relationship between representative parameters and connectivity; and (3) the need for increased attention to generating realistic conductivity fields, conditioned by geological information. Generation of geologically based conductivity fields has been a fertile field. However, it has grown relatively independent of the stochastically based literature, except 
for some preliminary results offered by the composite media approach. The time is probably ripe for the two fields to march together.

\section{NOTATION}

B domain thickness [L].

$\mathrm{C}_{\mathrm{a}}$ covariance function of SRF a.

e anisotropy ratio $\left(e=\lambda_{3} / \lambda_{1}\right.$ or $\left.\lambda_{2} / \lambda_{1}\right)$.

erf, erfc error function and complementary error function.

$\mathrm{E}(\mathbf{x})$ energy dissipation function.

$\mathrm{E}_{1}$ (L) Exponential integral function.

$f(\mathbf{x})$ source term.

$\mathrm{G}\left(\mathbf{x}, \mathbf{x}^{\prime}\right)$ deterministic Green's function.

$\mathrm{G}_{\mathrm{r}}\left(\mathbf{x}, \mathbf{x}^{\prime}\right)$ random Green's function.

$\underset{\widetilde{h}}{h}$ hydraulic head [L].

$\widetilde{h}$ hydraulic heads computed at the coarse-scale problem.

$\mathrm{H}$ Hurst coefficient.

$\mathrm{I}_{\mathrm{Y}}$ integral scale of log hydraulic conductivity [L].

$\mathrm{I}_{\mathrm{i}}$ directional integral scale of $\log$ hydraulic conductivity [L].

I identity tensor.

J mean hydraulic gradient [ ].

$\mathbf{k}$ wave vector $\left(\mathrm{k}_{1}, \mathrm{k}_{2}, \mathrm{k}_{3}\right)$ in spectral space.

$\mathrm{K}, \mathbf{K}$ hydraulic conductivity (either scalar or tensor) $\left[\mathrm{L} \mathrm{T}^{-1}\right]$.

$\mathrm{K}_{\mathrm{A}}$ arithmetic mean of the hydraulic conductivity values $\left[\mathrm{L} \mathrm{T}^{-1}\right]$.

$\mathrm{K}_{\mathrm{G}}$ geometric mean of the hydraulic conductivity values $\left[\mathrm{L} \mathrm{T}^{-1}\right]$.

$\mathrm{K}_{\mathrm{H}}$ harmonic mean of the hydraulic conductivity values $\left[\mathrm{L} \mathrm{T}^{-1}\right]$.

$\mathbf{K}_{\mathrm{b}}$ tensor of block-scale (or upscaled) hydraulic conductivity $\left[\mathrm{L} \mathrm{T}^{-1}\right]$.

$\mathbf{K}_{\text {eff }}$ tensor of effective (or pseudoeffective) hydraulic conductivity $\left[\mathrm{L} \mathrm{T}^{-1}\right]$.

$\mathrm{K}_{\text {eff,i }}$ effective (or pseudoeffective) hydraulic conductivity along direction $\mathrm{i}(\mathrm{i}=1,2,3)\left[\mathrm{L} \mathrm{T}^{-1}\right]$.

$\mathrm{K}_{\text {eff,ss }}$ effective (or pseudoeffective) hydraulic conductivity under steady state $\left[\mathrm{L} \mathrm{T}^{-1}\right]$.

$\mathrm{K}_{\mathrm{eq}}$ equivalent hydraulic conductivity [ $\left.\mathrm{L} \mathrm{T}^{-1}\right]$.

$\mathrm{K}_{\text {int }}$ interpreted hydraulic conductivity $\left[\mathrm{L} \mathrm{T}^{-1}\right]$.

$\mathrm{K}_{\mathrm{w}}$ hydraulic conductivity at the well location [L $\mathrm{T}^{-1}$ ].

$\mathbf{K}_{\mathbf{c}}(\mathbf{x})$ spatially varying conditional pseudoeffective

hydraulic conductivity tensor $\left[\mathrm{L} \mathrm{T}^{-1}\right]$.

$\tilde{\mathrm{K}}_{\mathrm{G}, \mathrm{ii}}$ upscaled hydraulic conductivity geometric mean $\left[\mathrm{L} \mathrm{T}^{-1}\right]$.

$\mathrm{L}$ finite size of domain [L].

$\mathrm{m}=\langle\mathrm{Y}\rangle$.

$\mathrm{n}$ problem dimensionality.

$p($ ) probability density function (pdf).

q vector of Darcy's velocity $\left[\mathrm{L} \mathrm{T}^{-1}\right]$.

Q pumping rate $\left[\mathrm{L}^{3} \mathrm{~T}^{-1}\right]$.

$\mathbf{r}$ residual flux vector $\left(\mathbf{r}=-\left\langle\mathrm{K}^{\prime} \nabla \mathrm{h}^{\prime}\right\rangle\right)$.

$r$ radial distance.

$\mathrm{r}_{\mathrm{w}}$ well radius [L].

$\mathrm{S}$ storativity $\left[\mathrm{L}^{-1}\right]$.

$\mathrm{S}_{\text {int }}$ interpreted storativity $\left[\mathrm{L}^{-1}\right]$.

$\mathrm{t}$ time [T].

$t^{*}$ dimensionless time.
$\mathrm{T}$ transmissivity $\left[\mathrm{L}^{2} \mathrm{~T}^{-1}\right]$.

$\mathrm{T}_{\mathrm{A}}$ arithmetic mean of the transmissivity values $\left[\mathrm{L}^{2}\right.$ $\left.\mathrm{T}^{-1}\right]$.

$\mathrm{T}_{\mathrm{G}}$ geometric mean of the transmissivity values $\left[\mathrm{L}^{2}\right.$ $\left.\mathrm{T}^{-1}\right]$.

$\mathrm{T}_{\mathrm{H}}$ harmonic mean of the transmissivity values $\left[\mathrm{L}^{2}\right.$ $\mathrm{T}^{-1}$ ].

$\mathrm{T}_{\text {eff }}$ effective (or pseudoeffective) transmissivity $\left[\mathrm{L}^{2}\right.$ $\mathrm{T}^{-1}$ ].

$\mathrm{T}_{\mathrm{eq}}, \mathrm{T}_{\mathrm{b}}$ equivalent transmissivity $\left[\mathrm{L}^{2} \mathrm{~T}^{-1}\right]$.

$\mathrm{T}_{\text {int }}$ interpreted transmissivity $\left[\mathrm{L}^{2} \mathrm{~T}^{-1}\right]$.

$\mathrm{T}_{\mathrm{w}}$ transmissivity at the well location $\left[\mathrm{L}^{2} \mathrm{~T}^{-1}\right]$.

$\mathrm{V}$ averaging volume $\left[\mathrm{L}^{3}\right]$.

$\mathbf{x}, \mathbf{x}_{\mathbf{i}}$ vector location [L].

$Z(\mathbf{x}, \omega)$ spatial random function.

Y natural logarithm of hydraulic conductivity or transmissivity.

$\gamma(\mathrm{s})$ semivariogram (function of separation distance, $s$ ).

$\delta()$ Dirac's delta function.

$\theta$ angle.

$\lambda$ isotropic correlation scale of $\mathrm{Y}[\mathrm{L}]$.

$\lambda_{\mathrm{i}}$ correlation scale along horizontal direction $\mathrm{x}_{\mathrm{i}}$ [L].

$\lambda_{\mathrm{H}} \quad$ harmonic average of correlation scales [L].

$\rho$ dimensionless flow domain size (measured in the flow direction); alternatively, normalized radial distance $(\rho=\mathrm{r} / \lambda)$.

$\hat{\rho}_{\mathrm{Y}}$ Fourier transform of the correlation function of Y.

$\sigma_{\mathrm{b}}^{2} \quad$ variance of random variable $\mathrm{b}$.

$\bar{\sigma}$ weighted average of the covariance function of Y.

$\xi$ dimensionless distance.

$\omega$ coordinate in probability space.

$\nabla$ gradient operator.

$\bar{a}$ spatial average of a.

$a^{\prime}$ random fluctuation (perturbation) of stochastic variable a.

$\mathrm{a}^{(\mathrm{i})} i$ th-order term in asymptotic expansion of a.

c subscript, indicating conditioning on measurements.

$\mathrm{T}$ superscript, transpose operator.

A large number of auxiliary variables and functions are used throughout the text. In such cases, definitions follow the first appearance. Angle brackets indicate expected value. Vertical bar indicates conditioning in probability space.

[234] ACKNOWLEDGMENTS. The Editor responsible for this paper was Daniel Tartakovsky. He thanks Shlomo P. Neuman, an anonymous technical reviewer, and an anonymous crossdisciplinary reviewer.

\section{REFERENCES}

Aasum, Y., E. Kasap, and M. Kelkar (1993), Analytical upscaling of small-scale permeability using a full tensor, SPE Pap. 25913, Soc. of Pet. Eng., Richardson, Tex.

Ababou, R. (1990), Identification of effective conductivity tensor in randomly heterogeneous and stratified aquifers, in Parameter Identification and Estimation for Aquifers and Reservoirs, 
edited by S. Bachu, pp. 155-157, Water Well J. Publ., Dublin, Ohio.

Ababou, R., and E. F. Wood (1990), Comment on "Effective groundwater model parameter values: Influence of spatial variability of hydraulic conductivity, leakance, and recharge" by J. J. Gomez-Hernandez and S. M. Gorelick, Water Resour. Res., 26(8), 1843-1846.

Abramovich, B., and P. Indelman (1995), Effective permittivity of log-normal isotropic random media, J. Phys. A Math. Gen., 28, 693-700.

Adler, P. M., and V. Mityushev (2003), Effective medium approximation and exact formulae for electrokinetic phenomena in porous media, J. Phys. A Math. Gen., 36, 391-404.

Alonso, E. E., and R. J. Krizek (1974), Randomness of settlement rate under stochastic load, J. Eng. Mech. Div. Am. Soc. Civ. Eng., 100(NEM6), 1211-1226.

Bakr, A. A., L. W. Gelhar, A. L. Gutjahr, and J. R. MacMillan (1978), Stochastic analysis of spatial variability in subsurface flows: 1. Comparison of one-dimensional and three-dimensional flows, Water Resour. Res., 14(2), 263-271.

Barker, J. A., and R. Herbert (1982), Pumping tests in patchy aquifers, Ground Water, 20(2), 150-155.

Batchelor, G. K. (1974), Transport properties of two-phase materials with random structure, Annu. Rev. Fluid Mech., 6, 227-255.

Beckie, R., and C. F. Harvey (2002), What does a slug test measure: An investigation of instrument response and the effects of heterogeneity, Water Resour. Res., 38(12), 1290, doi:10.1029/ 2001WR001072.

Beckie, R., A. A. Aldama, and E. F. Wood (1994), The universal structure of the groundwater flow equation, Water Resour. Res., 30(5), 1407-1419.

Begg, S. H., and P. R. King (1985), Modeling of the effects of shales on reservoir performance: Calculation of effective vertical permeability, paper presented at 8th Symposium on Reservoir Simulation, Soc. of Pet. Eng., Dallas, Tex.

Begg, S. H., D. M. Chang, and H. H. Haldorsen (1985), A simple statistical method of calculating the effective vertical permeability of a reservoir containing discontinuous shales, paper presented at 60th Annual Technical Conference, Soc. of Pet. Eng., Las Vegas, Nev.

Benson, C. H., H. M. Zhai, and S. M. Rashad (1994), Statistical sample-size for construction of soil liners, J. Geotech. Eng., 120(10), 1704-1724.

Bergman, D. J., and D. G. Stroud (2000), High-field magnetotransport in composite conductors: Effective-medium approximation, Phys. Rev. B, 62(10), 6603-6613.

Bertin, H., M. Panfilov, and M. Quintard (2000), Two types of transient phenomena and full relaxation macroscale model for single phase flow through double porosity media, Transp. Porous Media, 39, 73-96.

Bierkens, M. F. P. (1996), Modeling hydraulic conductivity of a complex confining layer at various spatial scales, Water Resour. Res., 32(8), 2369-2382.

Bierkens, M. F. P., and H. J. T. Weerts (1994), Block hydraulic conductivity of cross-bedded fluvial sediments, Water Resour. Res., 30(10), 2665-2678.

Bøe, Ø. (1994), Analysis of an upscaling method based on conservation of dissipation, Transp. Porous Media, 17, 77-86.

Boomsma, K., and D. Poulikakos (2001), On the effective thermal conductivity of a three dimensional structured fluid saturated metal foam, Int. J. Heat Mass Transfer, 44(4), 827-836.

Brace, W. F. (1984), Permeability of crystalline rocks: New in situ measurements, J. Geophys. Res., 89(B6), 4327-4330.

Bradbury, K. R., and M. A. Muldoon (1990), Hydraulic conductivity determinations in unlithified glacial and fluvial materials, in Ground Water and Vadose Zone Monitoring, pp. 138-151, Am. Soc. for Test. Mater., Philadelphia, Pa.

Bredehoeft, J. D., C. E. Neuzil, and P. C. D. Milly (1983), Regional flow in the Dakota aquifer: A study of the role of confining layers, U.S. Geol. Surv. Water Supply Pap., 2237, 1-45.
Bulgadaev, S. A. (2003), Duality and effective conductivity of random two-phase flat systems, Phys. Lett. A, 313(1-2), 106111

Bumgardner, J. D. (1990), Characterization of effective hydraulic conductivity in sand-clay mixtures, Master's thesis, Univ. of Calif., Davis.

Butler, J. J., Jr. (1988), Pumping tests in nonuniform aquifers-The radially symmetric case, J. Hydrol., 101, 15-30.

Butler, J. J., Jr. (1990), The role of pumping tests in site characterization: Some theoretical considerations, Ground Water, 28(3), 394-402.

Butler, J. J., Jr., and J. M. Healey (1998), Authors' reply, Ground Water, 36(6), 867-868, doi:10.1111/j.1745-6584.1998. tb02,092.x.

Butler, J. J., Jr., and W. Liu (1993), Pumping tests in nonuniform aquifers: The radially asymmetric case, Water Resour. Res., 29(2), 259-269.

Caldimi, V. V., and R. L. Mahajan (1999), The effective thermal conductivity of high porosity fibrous metal foams, J. Heat Transfer, 121, 466-471.

Cardwell, W. T., and R. L. Parsons (1945), Averaging permeability of heterogeneous oil sands, Trans. Am. Inst. Min. Metall. Pet. Eng., 160, 34-42.

Carrera, J., J. Heredia, S. Vomvoris, and P. Hufschmied (1990), Modeling of a flow on a small fractured monzonitic gneiss block, in Hydrogeology of Low Permeability Environments, Hydrol. Selec. Pap., vol. 2, pp. 115-169, H. Heise, Hannover, Germany. Chen, Y., L. J. Durlofsky, M. Gerritsen, and X. H. Wen (2003), A coupled local-global upscaling approach for simulating flow in highly heterogeneous formations, Adv. Water Resour., 26, 10411060 .

Christakos, G., D. T. Hristopulos, and C. Miller (1995), Stochastic diagrammatic analysis of groundwater flow in heterogeneous porous media, Water Resour. Res., 31(7), 1687-1703.

Clauser, C. (1992), Permeability of crystalline rocks, Eos Trans. $A G U, 73(21), 233,237-238$.

Clifton, P. M., and S. P. Neuman (1982), Effects of kriging and inverse modeling on conditional simulation of the Avra Valley aquifer in southern Arizona, Water Resour. Res., 18(4), 12151234.

Cooper, H. H., Jr., and C. E. Jacob (1946), A generalized graphical method for evaluating formation constants and summarizing well-field history, Eos Trans. AGU, 27(4), 526-534.

Copty, N. K., and A. N. Findikakis (2004), Stochastic analysis of pumping test drawdown data in heterogeneous geologic formations, J. Hydraul. Res., 42, 59-67.

Cushman, J. H. (1986), On measurement, scale, and scaling, Water Resour. Res., 22(2), 129-134.

Cushman, J. H., L. S. Bennethum, and B. X. Hu (2002), A primer on upscaling tools for porous media, Adv. Water Resour., 25(812), $1043-1067$.

Dagan, G. (1976), Comment on “A stochastic-conceptual analysis of one-dimensional groundwater flow in nonuniform homogeneous media" by R. A. Freeze, Water Resour. Res., 12(3), 567. Dagan, G. (1979), Models of groundwater flow in statistically homogeneous porous formations, Water Resour. Res., 15(1), $47-63$.

Dagan, G. (1981), Analysis of flow through heterogeneous random aquifers by the method of embedding matrix: 1. Steady flow, Water Resour. Res., 17(1), 107-121.

Dagan, G. (1982), Analysis of flow through heterogeneous random aquifers: 2 . Unsteady flow in confined formations, Water Resour. Res., 18(5), 1571-1585.

Dagan, G. (1986), Statistical theory of groundwater flow and transport: Pore to laboratory, laboratory to formation, and formation to regional scale, Water Resour. Res., 22(9), 120S-134S.

Dagan, G. (1987), Theory of solute transport by groundwater, Annu. Rev. Fluid Mech., 19, 183-215.

Dagan, G. (1989), Flow and Transport in Porous Formations, 465 pp., Springer, New York. 
Dagan, G. (1993), Higher-order correction of effective permeability of heterogeneous isotropic formations of lognormal conductivity distribution, Transp. Porous Media, 12, 279-290.

Dagan, G. (1997), Stochastic modeling of flow and transport: The broad perspective, in Subsurface Flow and Transport, edited by G. Dagan and S. P. Neuman, pp. 3-19, Cambridge Univ. Press, New York.

Dagan, G. (2001), Effective, equivalent and apparent properties of heterogeneous media, in Mechanics for a New Millennium, edited by H. Aref and J. W. Phillips, pp. 473-485, Springer, New York.

Danquigny, C., P. Ackerer, and J. P. Carlier (2004), Laboratory tracer tests on three-dimensional reconstructed heterogeneous porous media, J. Hydrol., 294, 196-212.

Darcy, H. (1856), Les fontaines publiques de la ville de Dijon, 647 pp., Dalmont, Paris.

Delhomme, J. P. (1979), Spatial variability and uncertainty in groundwater flow parameters: A geostatistical approach, Water Resour. Res., 15(2), 269-280.

Desbarats, A. J. (1987), Numerical estimation of effective permeability in sand-shale formations, Water Resour. Res., 23(2), 273-286.

Desbarats, A. J. (1990), Macrodispersion in sand-shale sequences, Water Resour. Res., 26(1), 153-163.

Desbarats, A. (1992), Spatial averaging of hydraulic conductivity in three-dimensional heterogeneous porous media, Math. Geol., 24(3), 249-267.

Desbarats, A. J. (1993), Geostatistical analysis of interwell transmissivity in heterogeneous aquifers, Water Resour. Res., 29(4), 1239-1246.

Desbarats, A. J. (1994), Spatial averaging of hydraulic conductivity under radial flow conditions, Math. Geol., 26(1), 1-21.

Desbarats, A. J., and S. Bachu (1994), Geostatistical analysis of aquifer heterogeneity from the core scale to the basin scale: A case study, Water Resour. Res., 30(3), 673-684.

Desbarats, A. J., and R. M. Srivastava (1991), Geostatistical characterization of groundwater-flow parameters in a simulated aquifer, Water Resour. Res., 27(5), 687-698.

Deutsch, C. V., and P. W. Cockerham (1994), Practical considerations in the application of simulated annealing to stochastic simulation, Math. Geol., 26(1), 67-82.

Deutsch, C. V., and A. Journel (1992), GSLIB: Geostatistical Software Library, Oxford Univ. Press, New York.

Deutsch, C. V., and T. T. Tran (2002), FLUVSIM: A program for object-based stochastic modeling of fluvial depositional systems, Comput. Geosci., 28, 525-535.

De Wit, A. (1995), Correlation structure dependence of the effective permeability of heterogeneous porous-media, Phys. Fluids, $7(11), 2553-2562$.

Di Federico, V., and S. P. Neuman (1997), Scaling of random fields by means of truncated power variograms and associated spectra, Water Resour. Res., 33(5), 1075-1085.

Di Federico, V., and S. P. Neuman (1998), Flow in multiscale log conductivity fields with truncated power variograms, Water Resour. Res., 34(5), 975-987.

Di Federico, V., S. P. Neuman, and D. M. Tartakovsky (1999), Anisotropy, lacunarity, upscaled conductivity and its covariance in multiscale fields with truncated power variograms, Water Resour. Res., 35(10), 2891-2908.

Du, C. Y., P. F. Shi, X. Q. Cheng, and G. P. Yin (2004), Effective protonic and electronic conductivity of the catalyst layers in proton exchange membrane fuel cells, Electrochem. Commun., $6(5), 435-440$.

Dupuit, J. (1863), Études théoretiques et pratiques sur le mouvement des eaux dans les canaux découverts et à travers les terrains perméables, Dunod, Paris.

Durlofsky, L. J. (1991), Numerical calculation of equivalent grid block permeability tensors for heterogeneous porous media, Water Resour. Res., 27(5), 699-708.

Durlofsky, L. J. (1992), Representation of grid block permeability in coarse scale models of randomly heterogeneous porous media, Water Resour. Res., 28(7), 1791-1800.
Durlofsky, L. J. (2000), An approximate model for well productivity in heterogeneous porous media, Math. Geol., 32(4), 421438.

Dykaar, B., and P. K. Kitanidis (1992), Determination of the effective hydraulic conductivity for heterogeneous porous media using a numerical spectral approach: 2. Results, Water Resour. Res., 28(4), 1167-1178.

Dykaar, B., and P. K. Kitanidis (1993), Transmissivity of a heterogeneous formation, Water Resour. Res., 29(4), 985-1001.

Eberhard, J., S. Attinger, and G. Wittum (2004), Coarse graining for upscaling of flow in heterogeneous porous media, Multiscale Model. Simul., 2(2), 269-301.

El-Kadi, A. I., and W. Brutsaert (1985), Applicability of effective parameters for unsteady flow in nonuniform aquifers, Water Resour. Res., 21(2), 183-198.

Farmer, C. L. (2002), Upscaling: A review, Int. J. Numer. Methods Fluids, 40, 63-78, doi:10.1002/fld.267.

Fenton, G. A., and D. V. Griffiths (1993), Statistics of block conductivity through a simple bounded stochastic medium, Water Resour. Res., 29(6), 1825-1830.

Flodin, E. A., L. J. Durlofsky, and A. Aydin (2004), Upscaled models of flow and transport in faulted sandstone: Boundary condition effects and explicit fracture modelling, Pet. Geosci., 10(2), $173-181$.

Fokin, A. G. (1996), Macroscopic conductivity of random inhomogeneous media: Calculation methods (in Russian), Usp. Fiz. Nauk, 166(10), 1069-1093.

Fokker, P. A. (2001), General anisotropic effective medium theory for the effective permeability of heterogeneous reservoirs, Transp. Porous Media, 44, 205-218.

Follin, S. (1992), Numerical calculations of heterogeneity of groundwater flow, Ph.D. dissertation, Dep. of Land and Water Resour., R. Inst. of Technol., Stockholm.

Fourier, J. B. J. (1822), Theorie Analytique de la Chaleur, F. Didot, Paris.

Freeze, R. A. (1975), A stochastic-conceptual analysis of onedimensional groundwater flow in nonuniform homogeneous media, Water Resour. Res., 11(5), 725-741.

Froidevaux, R. (1993), Probability field simulation, in Geostatistics Troia'92, edited by A. Soares, pp. 73-84, Springer, New York.

Gautier, Y., and B. Noetinger (1997), Preferential flow-paths detection for heterogeneous reservoirs using a new renormalization technique, Transp. Porous Media, 26, 1-23.

Gelhar, L. W. (1986), Stochastic subsurface hydrology: From theory to applications, Water Resour. Res., 22(9), 135S-145S.

Gelhar, L. W. (1993), Stochastic Subsurface Hydrology, 390 pp., Prentice-Hall, Upper Saddle River, N. J.

Gelhar, L. W., and C. L. Axness (1983), Three-dimensional stochastic-analysis of macrodispersion in aquifers, Water Resour. Res., 19(1), 161-180.

Gelhar, L. W., A. A. Bakr, A. L. Gutjahr, and J. R. MacMillan (1977), Comments on "A stochastic-conceptual analysis of onedimensional groundwater flow in nonuniform homogeneous media" by R. Allan Freeze, Water Resour. Res., 13(2), 477479.

Gómez-Hernández, J. J. (1991), A stochastic approach to the simulation of conductivity fields conditioned upon data measured at a smaller scale, Ph.D. dissertation, Dep. Appl. Sci., Stanford Univ., Stanford, Calif.

Gómez-Hernández, J. J., and S. M. Gorelick (1989), Effective groundwater model parameter values: Influence of spatial variability of hydraulic conductivity, leakance, and recharge, Water Resour. Res., 25(3), 405-419.

Gómez-Hernández, J. J., and R. M. Srivastava (1990), ISIM3D: An ANSI-C three-dimensional multiple indicator conditional simulation program, Comput. Geosci., 16, 395-440.

Gómez-Hernández, J. J., and X.-H. Wen (1998), To be or not to be multiGaussian? A reflection on stochastic hydrogeology, $A d v$. Water Resour., 21, 47-61. 
Gómez-Hernández, J. J., H. Sovero, and A. Sahuquillo (1995), Some issues on the analysis of pumping tests in heterogeneous aquifers, Hydrogeologie, 3, 13-18.

Greenberg, M. D. (1971), Application of Green's Functions in Science and Engineering, Prentice-Hall, Upper Saddle River, N. J.

Guadagnini, A., and S. P. Neuman (1999a), Nonlocal and localized analyses of conditional mean steady state flow in bounded, randomly nonuniform domains: 1 . Theory and computational approach, Water Resour. Res., 35(10), 2999-3018.

Guadagnini, A., and S. P. Neuman (1999b), Nonlocal and localized analyses of conditional mean steady state flow in bounded, randomly nonuniform domains: 2. Computational examples, Water Resour. Res., 35(10), 3019-3039.

Guadagnini, A., M. Riva, and S. P. Neuman (2003), Threedimensional steady state flow to a well in a randomly heterogeneous bounded aquifer, Water Resour. Res., 39(3), 1048, doi:10.1029/2002WR001443.

Guérillot, D., J. L. Rudkiewicz, C. Ravenne, G. Renard, and A. Galli (1990), An integrated model for computer-aided reservoir description: From outcrop study to fluid-flow simulations, Rev. Inst. Fr. Pet., 45(1), 71-77.

Guimerà, J., L. Vives, and J. Carrera (1995), A discussion of scale effects on hydraulic conductivity at a granitic site (El Berrocal, Spain), Geophys. Res. Lett., 22(11), 1449-1452.

Gutjahr, A. L., L. W. Gelhar, A. A. Bakr, and J. R. MacMillan (1978), Stochastic analysis of spatial variability in subsurface flows: 2. Evaluation and application, Water Resour. Res., 14(5), 953-959.

Hastings, J. J., and A. H. Muggeridge (2001), Upscaling uncertain permeability using small cell renormalization, Math. Geol., 33(4), 491-505.

He, C. P., M. G. Edwards, and L. J. Durlofsky (2002), Numerical calculation of equivalent cell permeability tensors for general quadrilateral control volumes, Comput. Geosci., 6, 28-47.

Hernandez, A. F., S. P. Neuman, A. Guadagnini, and J. Carrera (2003), Conditioning mean steady state flow on hydraulic head and conductivity through geostatistical inversion, Stochastic Environ. Res. Risk Assess., 17, 329-338, doi:10.1007/s00477-0030154-4.

Hersvik, K. J., and M. S. Espedal (1998), Adaptive hierarchical upscaling of flow in heterogeneous reservoirs based on an a posteriori error estimate, Comput. Geosci., 2, 311-336.

Hoeksema, R. J., and P. K. Kitanidis (1985), Analysis of the spatial structure of properties of selected aquifers, Water Resour. Res., 21(4), 563-572.

Holden, L., and B. F. Nielsen (2000), Global upscaling of permeability in heterogeneous reservoirs: The output least squares (OLS) method, Transp. Porous Media, 40, 115-143.

Hristopulos, D. T., and G. Christakos (1997), Variational calculation of the effective fluid permeability of heterogeneous media, Phys. Rev. E, 55(6), 7288-7298.

Hristopulos, D. T., and G. Christakos (1999), Renormalization group analysis of permeability upscaling, Stochastic Environ. Res. Risk Assess., 13(1-2), 131-160.

Illman, W. A. (2004), Analysis of permeability scaling within single boreholes, Geophys. Res. Lett., 31(5), L06503, doi:10.1029/ 2003GL019303.

Illman, W. A., and S. P. Neuman (2001), Type curve interpretation of a cross-hole pneumatic injection test in unsaturated fractured tuff, Water Resour. Res., 37(3), 583-603.

Illman, W. A., and S. P. Neuman (2003), Steady-state analysis of cross-hole pneumatic injection tests in unsaturated fractured tuff, J. Hydrol., 281, 36-54.

Indelman, P. (1996), Averaging of unsteady flows in heterogeneous media of stationary conductivity, J. Fluid Mech., 310, 39-60.

Indelman, P. (2000), Unsteady source flow in weakly heterogeneous porous media, Comput. Geosci., 4, 351-381.

Indelman, P. (2001), Steady-state source flow in heterogeneous porous media, Transp. Porous Media, 45, 105-127.
Indelman, P. (2003a), Transient well-type flows in heterogenous formations, Water Resour. Res., 39(3), 1064, doi:10.1029/ 2002WR001407.

Indelman, P. (2003b), Transient pumping well flow in weakly heterogeneous formations, Water Resour. Res., 39(10), 1287, doi:10.1029/2003WR002036.

Indelman, P., and B. Abramovich (1994a), A higher-order approximation to effective conductivity in media of anisotropic random structure, Water Resour. Res., 30(6), 1857-1864.

Indelman, P., and B. Abramovich (1994b), Nonlocal properties of nonuniform averaged flow in heterogeneous media, Water Resour. Res., 30(12), 3385-3393.

Indelman, P., and G. Dagan (1993a), Upscaling of conductivity of heterogeneous formations: General approach and application to isotropic media, Transp. Porous Media, 12, 161-183.

Indelman, P., and G. Dagan (1993b), Upscaling of permeability of anisotropic heterogeneous formations: 1 . The general framework, Water Resour. Res., 29(4), 917-923.

Indelman, P., and G. Dagan (1993c), Upscaling of permeability of anisotropic heterogeneous formations: 2. General structure and small perturbation analysis, Water Resour. Res., 29(4), 925-933.

Indelman, P., and Y. Rubin (1995), Flow in heterogeneous media displaying a linear trend in the log conductivity, Water Resour. Res., 31(5), 1257-1266.

Indelman, P., and Y. Rubin (1996), Average flow in heterogeneous media of trending hydraulic conductivity, J. Hydrol., 183, 5768.

Indelman, P., A. Fiori, and G. Dagan (1996), Steady flow toward wells in heterogeneous formations: Mean head and equivalent conductivity, Water Resour. Res., 32(7), 1975-1983.

Jankovic, I., A. Fiori, and G. Dagan (2003), Effective conductivity of an isotropic heterogeneous medium of lognormal conductivity distribution, Multiscale Model. Simul., 1(1), 40-56.

Jensen, J. L. (1991), Use of the geometric average for effective permeability estimation, Math. Geol., 23(6), 833-840.

Journel, A. G., and C. V. Deutsch (1993), Entropy and spatial disorder, Math. Geol., 25(3), 329-355.

Journel, A. G., and C. J. Huijbregts (1978), Mining Geostatistics, Elsevier, New York.

Journel, A. G., C. V. Deutsch, and A. J. Desbarats (1986), Power averaging for block effective permeability, paper presented at 56th California Regional Meeting, Soc. of Pet. Eng., Oakland, Calif.

Jussel, P., F. Stauffer, and T. Dracos (1994a), Transport modeling in heterogeneous aquifers: 1 . Statistical description and numerical generation of gravel deposits, Water Resour. Res., 30(6), $1803-1817$.

Jussel, P., F. Stauffer, and T. Dracos (1994b), Transport modeling in heterogeneous aquifers: 2 . Three-dimensional transport model and stochastic numerical tracer experiments, Water Resour. Res. 30(6), 1819-1831.

Kacimov, A. R., Y. V. Obnosov, and N. D. Yakimov (1999), Groundwater flow in a medium with a parquet-type conductivity distribution, J. Hydrol., 226, 242-249.

Kaganova, I. M. (2003), On calculation of effective conductivity of inhomogeneous metals, Phys. Lett. A, 312(1-2), 108-118.

Kaganova, I. M., and M. I. Kaganov (2004), Calculating effective galvanomagnetic characteristics of inhomogeneous metals: Exact solution for polycrystals of metals in high magnetic fields, Laser Phys., 14(3), 416-425.

Kasap, E. L., and L. W. Lake (1990), Calculating the effective permeability tensor of a gridblock, SPE Form. Eval., 5(2), $192-200$

King, P. R. (1988), The use of renormalization for calculating effective permeability, Transp. Porous Media, 4, 37-58.

Kitanidis, P. K. (1990), Effective conductivity for gradually varying flow, Water Resour. Res., 26(6), 1197-1208.

Knudby, C. J. (2004), On the concept of hydrogeologic connectivity, Ph.D. thesis, 104 pp., Univ. Politècnica de Catalunya, Barcelona. 
Knudby, C., and J. Carrera (2005), On the relationship between indicators of geostatistical, flow and transport connectivity, $A d v$. Water Resour., 28, 405-421.

Koltermann, C., and S. M. Gorelick (1996), Heterogeneity in sedimentary deposits: A review of structure-imitating, processimitating, and descriptive approaches, Water Resour. Res., 32(9), 2617-2658.

Kraichnan, R. H. (1987), Eddy viscosity and diffusivity: Exact formulas and approximations, Complex Syst., 1, 805-820.

Landau, L. D., and E. M. Lifshitz (1960), Electrodynamics of Continuous Media, Elsevier, New York.

Lee, J. Y., and K. K. Lee (1999), Analysis of the quality of parameter estimates from repeated pumping and slug tests in a fractured porous aquifer system in Wonju, Korea, Ground Water, 37(5), 692-700.

Le Loc'h, G. (1987), Étude de la composition des perméabilités par des methods variationelles, Ph.D. thesis, Paris Sch. of Mines, Paris.

Li, S.-G., and D. McLaughlin (1995), Using the nonstationary spectral method to analyze flow through heterogeneous trending media, Water Resour. Res., 31(3), 541-552.

Loaiciga, H. A., R. B. Leipnik, M. A. Mariño, and P. F. Hudak (1993), Stochastic groundwater flow analysis in the presence of trends in heterogeneous hydraulic conductivity fields, Math. Geol., 25(2), 161-176.

Loaiciga, H. A., R. B. Leipnik, P. F. Hudak, and M. A. Mariño (1994), Effective hydraulic conductivity of nonstationary aquifers, Stochastic Hydrol. Hydraul., 8(1), 1-17.

Loaiciga, H. A., R. B. Leipnik, P. F. Hudak, and M. A. Mariño (1996), 1-, 2-, and 3-dimensional effective conductivity of aquifers, Math. Geol., 28(5), 563-584.

Lough, M. F., S. H. Lee, and J. Kamath (1997), A new method to calculate effective permeability of gridblocks used in the simulation of naturally fractured reservoirs, SPE Reservoir Eng., 12(3), 219-224.

Lu, Z., and D. Zhang (2002), On stochastic modeling of flow in multimodal heterogeneous formations, Water Resour. Res., 38(10), 1190, doi:10.1029/2001WR001026.

Madden, T. R. (1976), Random networks and mixing laws, Geophysics, 41(6A), 1104-1125.

Mandelbrot, B. B., and J. W. Van Ness (1968), Fractional Brownian motions, fractional noises and applications, SIAM Rev., 10, $422-437$.

Mantoglou, A., and J. L. Wilson (1982), The turning bands method for simulation of random fields using line generation by a spectral method, Water Resour. Res., 18(5), 1379-1394.

Martinez-Landa, L., and J. Carrera (2005), An analysis of hydraulic conductivity scale effects in granite (Full-scale Engineered Barrier Experiment (FEBEX), Grimsel, Switzerland), Water Resour. Res., 41(3), W03006, doi:10.1029/2004WR003458.

Matheron, G. (1965), Les variables régionalisées et leur estimation, Masson, Paris.

Matheron, G. (1967), Eléments pour une Théorie des Milieux Poruex, Masson, Paris.

Matheron, G., H. Beucher, C. de Fouquet, A. Galli, D. Guerillot, and C. Ravenne (1987), Conditional simulation of the geometry of fluvio-deltaic reservoirs, SPE Pap. 16753, Soc. of Pet. Eng., Richardson, Tex.

McCarthy, J. F. (1990), Effective permeability of sandstone shale reservoirs by a random-walk method, J. Phys. A Math. Gen., 23, L445-L451.

McCarthy, J. F. (1991), Analytical models of the effective permeability of sand-shale reservoirs, Geophys. J. Int., 105(2), 513527.

McCarthy, J. F. (1993), Continuous-time random-walks on randommedia, J. Phys. A Math. Gen., 26, 2495-2503.

Meier, P. M., J. Carrera, and X. Sanchez-Vila (1998), An evaluation of Jacob's method for the interpretation of pumping tests in heterogeneous formations, Water Resour. Res., 34(5), 10111025 .
Meier, P. M., J. Carrera, and X. Sanchez-Vila (1999), A numerical study on the relationship between transmissivity and specific capacity in heterogeneous aquifers, Ground Water, 37(4), 611617.

Mizell, S. A., A. L. Gutjahr, and L. W. Gelhar (1982), Stochastic analysis of spatial variability in two-dimensional steady groundwater flow assuming stationary and nonstationary heads, Water Resour. Res., 18(4), 1053-1067.

Naff, R. L. (1991), Radial flow in heterogeneous porous media: An analysis of specific discharge, Water Resour. Res., 27(3), 307316.

Naff, R. L., D. F. Haley, and E. A. Sudicky (1998), High-resolution Monte Carlo simulation of flow and conservative transport in heterogeneous porous media: 1. Methodology and flow results, Water Resour. Res., 34(4), 663-677.

Narasimhan, T. N. (1999), Fourier's heat conduction equation: History, influence, and connections, Rev. Geophys., 37(1), $151-172$.

Neuman, S. P. (1990), Universal scaling of hydraulic conductivities and dispersivities in geologic media, Water Resour. Res., 26(8), $1749-1758$.

Neuman, S. P. (1994), Generalized scaling of permeabilities: Validation and effect of support scale, Geophys. Res. Lett., 21(5), $349-352$.

Neuman, S. P. (2003), Relationship between juxtaposed, overlapping, and fractal representations of multimodal spatial variability, Water Resour. Res., 39(8), 1205, doi:10.1029/2002WR001755.

Neuman, S. P., and V. Di Federico (2003), Multifaceted nature of hydrologic scaling and its interpretation, Rev. Geophys., 41(3), 1014, doi:10.1029/2003RG000130.

Neuman, S. P., and A. Guadagnini (2000), A new look at traditional deterministic flow models and their calibration in the context of randomly heterogeneous media, IAHS Publ., 265, $213-221$.

Neuman, S. P., and S. Orr (1993), Prediction of steady state flow in nonuniform geologic media by conditional moments: Exact nonlocal formalism, effective conductivities, and weak approximation, Water Resour. Res., 29(2), 341-364.

Neuman, S. P., D. M. Tartakovsky, T. C. Wallstrom, and C. L. Winter (1996), Correction to "Prediction of steady state flow in nonuniform geologic media by conditional moments: Exact nonlocal formalism, effective conductivities, and weak approximation" by Shlomo P. Neuman and Shloomo Orr, Water Resour. Res., 32(5), 1479-1480.

Neuman, S. P., A. Guadagnini, and M. Riva (2004), Type-curve estimation of statistical heterogeneity, Water Resour. Res., 40, W04201, doi:10.1029/2003WR002405.

Nielsen, B. F., and A. Tveito (1998), An upscaling method for onephase flow in heterogeneous reservoirs: A weighted output least squares (WOLS) approach, Comput. Geosci., 2, 93-123.

Nilsen, S., and M. S. Espedal (1996), Wavelet upscaling based on piecewise bilinear approximation of the permeability field, Transp. Porous Media, 23, 125-134.

Nobes, D. C. (1996), Troubled waters: Environmental applications of electrical and electromagnetic methods, Surv. Geophys., 17(4), $393-454$.

Noetinger, B. (1994), The effective permeability of a heterogeneous porous-medium, Transp. Porous Media, 15, 99-127.

Noetinger, B. (2000), Computing the effective permeability of lognormal permeability fields using renormalization methods, $C$. $R$. Acad. Sci., Ser. IIa Sci. Terre Planetes, 331(5), 353-357.

Noetinger, B., and T. Estebenet (2000), Up-scaling of double porosity fractured media using continuous-time random walks methods, Transp. Porous Media, 39, 315-337.

Noetinger, B., and Y. Gautier (1998), Use of the Fourier-Laplace transform and of diagrammatical methods to interpret pumping tests in heterogeneous reservoirs, Adv. Water Resour., 21, 581590.

Oliver, D. S. (1990), The averaging process in permeability estimation from well-test data, Trans. Soc. Pet. Eng., 289, 319-324. 
Oliver, L. D., and G. Christakos (1996), Boundary condition sensitivity analysis of the stochastic flow equation, $A d v$. Water Resour., 19(2), 109-120.

Owhadi, H. (2003), Approximation of the effective conductivity of ergodic media by periodization, Probab. Theory Related Fields, 125(2), 225-258.

Paleologos, E. K. (1994), Effective hydraulic conductivity of bounded, strongly heterogeneous porous media, Ph.D. dissertation, 198 pp., Univ. of Ariz., Tucson.

Paleologos, E. K., S. P. Neuman, and D. Tartakovsky (1996), Effective hydraulic conductivity of bounded, strongly heterogeneous porous media, Water Resour. Res., 32(5), 13331341.

Panda, M. N., C. C. Mosher, and A. K. Chopra (2000), Application of wavelet transforms to reservoir-data analysis and scaling, SPE $J ., 5(1), 92-101$.

Phillips, S. P., and K. Belitz (1991), Calibration of a texture-based model of a groundwater-flow system, western San Joaquin Valley, California, Ground Water, 29(5), 702-715.

Pickup, G. E., and C. Y. Hern (2002), The development of appropriate upscaling procedures, Transp. Porous Media, 46, 119138.

Pickup, G. E., P. S. Ringrose, J. L. Jensen, and K. S. Sorbie (1994), Permeability tensors for sedimentary structures, Math. Geol., 26(2), 227-250.

Poeter, E., and P. Townsend (1994), Assessment of critical flow path for improved remediation management, Ground Water, $32(3), 439-447$.

Poley, A. D. (1988), Effective permeability and dispersion in locally heterogeneous aquifers, Water Resour. Res., 24(11), 19211926.

Pozdniakov, S., and C.-F. Tsang (1999), A semianalytical approach to spatial averaging of hydraulic conductivity in heterogeneous aquifers, J. Hydrol., 216, 78-98.

Pozdniakov, S., and C.-F. Tsang (2004), A self-consistent approach for calculating the effective hydraulic conductivity of a binary, heterogeneous medium, Water Resour. Res., 40(5), W05105, doi:10.1029/2003WR002617.

Raghavan, R. (2004), A review of applications to constrain pumping test responses to improve on geological description and uncertainty, Rev. Geophys., 42, RG4001, doi:10.1029/ 2003RG000142.

Renard, P., and G. de Marsily (1997), Calculating equivalent permeability: A review, Adv. Water Resour., 20, 253-278.

Renard, P., G. Le Loc'h, E. Ledoux, G. de Marsily, and R. Mackay (2000), A fast algorithm for the estimation of the equivalent hydraulic conductivity of heterogeneous media, Water Resour. Res., 36(12), 3567-3580.

Revelli, R., and L. Ridolfi (2000), Influence of heterogeneity on the flow in unconfined aquifers, J. Hydrol., 228, 150-159.

Riva, M., A. Guadagnini, S. P. Neuman, and S. Franzetti (2001), Radial flow in a bounded randomly heterogeneous aquifer, Transp. Porous Media, 45, 139-193.

Romeu, R. K. (1994), Écoulement en milieux hétérogènes: Prise de moyenne de perméabilité en régimes permanent et transitoire, Ph.D. thesis, 286 pp., Univ. Paris VI, Paris.

Rovey, C. W. (1998), Digital simulation of the scale effect in hydraulic conductivity, Hydrogeol. J., 6(2), 216-225.

Rovey, C. W., and D. S. Cherkauer (1995), Scale dependency of hydraulic conductivity measurements, Ground Water, 33(5), $769-780$.

Rovey, C. W., and W. L. Niemann (2001), Wellskins and slug tests: Where's the bias?, J. Hydrol., 243, 120-132.

Rubin, Y. (1995), Flow and transport in bimodal heterogeneous formations, Water Resour. Res., 31(10), 2461-2468.

Rubin, Y. (2003), Applied Stochastic Hydrogeology, 391 pp., Oxford Univ. Press, New York.

Rubin, Y., and G. Dagan (1987a), Stochastic identification of transmissivity and effective recharge in steady groundwater flow: 1 . Theory, Water Resour. Res., 23(7), 1185-1192.
Rubin, Y., and G. Dagan (1987b), Stochastic identification of transmissivity and effective recharge in steady groundwater flow: 2 . Case study, Water Resour. Res., 23(7), 1193-1200.

Rubin, Y., and J. J. Gómez-Hernández (1990), A stochastic approach to the problem of upscaling of conductivity in disordered media: Theory and unconditional numerical simulations, Water Resour. Res., 26(4), 691-701.

Rubin, Y., and K. Seong (1994), Investigation of flow and transport in certain cases of nonstationary conductivity fields, Water Resour. Res., 30(11), 2901-2912.

Rubinstein, J., and S. Torquato (1989), Flow in random porous media: Mathematical formulation, variational principles and rigorous bounds, J. Fluid Mech., 206, 25-46.

Samper, J., and J. Carrera (1990), Geoestadistica: Aplicaciones a la hidrología subterranean, 484 pp., Int. Cent. for Numer. Methods in Eng., Barcelona.

Samper-Calvete, F. J., and M. A. García-Vera (1998), Inverse modeling of groundwater flow in the semiarid evaporitic closed basin of Los Monegros, Spain, Hydrogeol. J., 6(1), 33-49.

Sanchez-Vila, X. (1997), Radially convergent flow in heterogeneous porous media, Water Resour. Res., 33(7), 1633-1641.

Sanchez-Vila, X., and J. Carrera (1997), Directional effects on convergent flow tracer tests, Math. Geol., 29(4), 551-569.

Sanchez-Vila, X., J. P. Girardi, and J. Carrera (1995), A synthesis of approaches to upscaling of hydraulic conductivities, Water Resour. Res., 31(4), 867-882.

Sanchez-Vila, X., J. Carrera, and J. P. Girardi (1996), Scale effects in transmissivity, J. Hydrol., 183, 1-22.

Sanchez-Vila, X., C. L. Axness, and J. Carrera (1999a), Upscaling transmissivity under radially convergent flow in heterogeneous media, Water Resour. Res., 35(3), 613-621.

Sanchez-Vila, X., P. M. Meier, and J. Carrera (1999b), Pumping tests in heterogeneous aquifers: An analytical study of what can be obtained from their interpretation using Jacob's method, Water Resour. Res., 35(4), 943-952.

Sarris, T. S., and E. K. Paleologos (2004), Numerical investigation of the anisotropic hydraulic conductivity behavior in heterogeneous porous media, Stochastic Environ. Res. Risk Assess., 18(3), 188-197.

Schad, H., and G. Teutsch (1994), Effects of the investigation scale on pumping tests results in heterogeneous porous aquifers, J. Hydrol., 159, 61-77.

Schulze-Makuch, D., D. A. Carlson, D. S. Cherkauer, and P. Malik (1999), Scale dependency of hydraulic conductivity in heterogeneous media, Ground Water, 37(6), 904-919.

Serrano, S. E. (1997), The Theis solution in heterogeneous aquifers, Ground Water, 25(3), 463-467.

Shrestha, S. P., and G. V. Loganathan (1994), Monte Carlo simulation and effective medium approximation in subsurface flow modeling, Ground Water, 32(6), 929-936.

Shvidler, M. I. (1962), Filtration flows in heterogeneous media (in Russian), Izv. Akad. Nauk SSSR Mekh. I Mas., 3, 185-190.

Shvidler, M. I. (1966), The source-type solution in the problem of unsteady filtration in a medium with random nonuniformity (in Russian), Izv. Akad. Nauk SSSR Ser. Mek. Zhidk. Gaza, 4, 137141. (English translation Fluid Dyn., 1(4), 95-98, 1966).

Shvidler, M. I. (1985), Stochastic Hydrodynamics of Porous Media (in Russian), Nedra, Moscow.

Shvidler, M. I. (1986), Conditional averaging of unsteady percolation fields in random composite porous media, Fluid Dyn., 21(5), $735-740$

Shvidler, M. I. (1988), Multicontinuum description of percolating flow through periodic inhomogeneous porous media, Fluid Dyn., 23(6), 894-901.

Silliman, S. E., and A. L. Wright (1988), Stochastic analysis of paths of high hydraulic conductivity in porous media, Water Resour. Res., 24(11), 1901-1910.

Smith, L., and R. A. Freeze (1979), Stochastic analysis of steady state groundwater flow in a bounded domain: 2 . Two-dimensional simulations, Water Resour. Res., 15(6), 1543-1559. 
Spector, M., and P. Indelman (1998), On second-order correction to the effective conductivity of two-dimensional anisotropic media, Water Resour. Res., 34(5), 1357-1359.

Stam, J. M. T., and W. Zijl (1992), Modeling permeability in imperfectly layered porous-media. 2. A 2-dimensional application of block-scale permeability, Math. Geol., 24(8), 885-904.

Stepanyants, Y. A., and E. V. Teodorovich (2003), Effective hydraulic conductivity of a randomly heterogeneous porous medium, Water Resour. Res., 39(3), 1065, doi:10.1029/2001WR000366.

Tartakovsky, D. M., and A. Guadagnini (2004), Effective properties of random composites, SIAM J. Sci. Comp., 26(2), 625-635.

Tartakovsky, D. M., and S. P. Neuman (1998a), Transient flow in bounded randomly heterogeneous domains: 2 . Localization of conditional mean equations and temporal nonlocality effects, Water Resour. Res., 34(1), 13-20.

Tartakovsky, D. M., and S. P. Neuman (1998b), Transient effective hydraulic conductivities under slowly and rapidly varying mean gradients in bounded three-dimensional random media, Water Resour. Res., 34(1), 21-32.

Tartakovsky, D. M., A. Guadagnini, and L. Guadagnini (2000), Effective hydraulic conductivity and transmissivity for heterogeneous aquifers, Math. Geol., 32(6), 751-759.

Tartakovsky, D. M., A. Guadagnini, F. Ballio, and A. M. Tartakovsky (2002), Localization of mean flow and apparent transmissivity tensor for bounded randomly heterogeneous aquifers, Transp. Porous Media, 49, 41-58.

Tegnander, C., and T. Gimse (1998), Flow simulations to evaluate upscaling of permeability, Math. Geol., 30(6), 717-731.

Telega, J. J., and W. R. Bielski (2003), Flows in random porous media: Effective models, Comput. Geotech., 30, 271-288.

Teles, V., F. Delay, and G. de Marsily (2004), Comparison of genesis and geostatistical methods for characterizing the heterogeneity of alluvial media: Groundwater flow and transport simulations, J. Hydrol., 294, 103-121.

Teodorovich, E. V. (1997), Calculation of the effective permeability of a randomly inhomogeneous porous medium, Sov. Phys. JETP, Engl. Transl., 85(1), 173-178.

Teodorovich, E. V. (2000), The effective conductivity of a randomly inhomogeneous medium, J. Appl. Math. Mech., 64(6), 951-957.

Terzaghi, K., and R. B. Peck (1948), Soil Mechanics in Engineering Practice, 729 pp., John Wiley, Hoboken, N. J.

Theis, C. V. (1935), The relation between the lowering of the piezometric surface and the rate and duration of discharge of a well using ground-water storage, Eos Trans. AGU, 16, 519-524.

Thiem, G. (1906), Hydrologische Methoden, Gebhardt, Leipzig, Germany.

Tidwell, V. C., and J. L. Wilson (1999a), Upscaling experiments conducted on a block of volcanic tuff: Results for a bimodal permeability distribution, Water Resources Res., 35(11), 33753387.

Tidwell, V. C., and J. L. Wilson (1999b), Permeability upscaling measured on a block of berea sandstone: Results and interpretation, Math. Geol., 31(7), 749-769.

Tidwell, V. C., and J. L. Wilson (2000), Heterogeneity, permeability patterns, and permeability upscaling: Physical characterization of a block of Massillon sandstone exhibiting nested scales of heterogeneity, SPE Reservoir Eval. Eng., 3(4), 283-291.

Tidwell, V. C., A. L. Gutjahr, and J. L. Wilson (1999), What does an instrument measure? Empirical spatial weighting functions calculated from permeability data sets measured on multiple sample supports, Water Resour. Res., 35(1), 43-54.

Tiedeman, C. R., P. A. Hsieh, and S. B. Christian (1995), Characterization of a high-transmissivity zone by well test analysis: Steady state case, Water Resour. Res., 31(1), 27-37.

Torquato, S. (2002), Random Heterogeneous Materials, 701 pp., Springer, New York.

Trykozko, A., and W. Zijl (2002), Complementary finite element methods applied to the numerical homogeneization of 3D absolute permeability, Commun. Numer. Methods Eng., 18, 31-41.
Vandenberg, A. (1977), Pump testing in heterogeneous aquifers, J. Hydrol., 34, 45-62.

van den Berg, E. H., and J. J. de Vries (2003), Influence of grain fabric and lamination on the anisotropy of hydraulic conductivity in unconsolidated dune sands, J. Hydrol., 283, 244-266.

Veneziano, D., and A. Tabaei (2001), Analysis of variance method for the equivalent conductivity of rectangular blocks, Water Resour. Res., 37(12), 2919-2927.

Vesselinov, V. V., S. P. Neuman, and W. A. Illman (2001), Threedimensional numerical inversion of pneumatic cross-hole tests in unsaturated fractured tuff: 2. Equivalent parameters, highresolution stochastic imaging, and scale effects, Water Resour. Res., 37(12), 3019-3041.

Vidstrand, P. (2001), Comparison of upscaled methods to estimate hydraulic conductivity, Ground Water, 39(3), 401-407.

Warren, J. E., and H. S. Price (1961), Flow in heterogeneous porous media, Soc. Pet. Eng. J., 1, 153-169.

Webb, E. K., and M. P. Anderson (1996), Simulation of preferential flow in three-dimensional, heterogeneous, conductivity fields with realistic internal architecture, Water Resour. Res., 32(3), $533-545$.

Wen, X.-H., and J. J. Gómez-Hernández (1996), Upscaling hydraulic conductivities in heterogeneous media: An overview, J. Hydrol., 183, ix-xxxii.

Wen, X. H., and J. J. Gómez-Hernández (1998), Upscaling hydraulic conductivities in cross-bedded formations, Math. Geol., 30(2), $181-211$.

Wen, X. H., L. J. Durlofsky, and M. G. Edwards (2003), Use of border regions for improved permeability upscaling, Math. Geol., 35(5), 521-547.

Wessel-Berg, D., and P. E. S. Bergmo (2002), A new exact upscaling formula and investigations of numerical errors in simulation of single- and two-phase Darcy flow in barrier geometries, Transp. Porous Media, 46, 233-249.

Whitaker, F. F., and P. L. Smart (1997), Climatic control of hydraulic conductivity of Bahamian limestones, Ground Water, 35(5), 859-868.

Winter, C. L., and D. M. Tartakovsky (2000), Mean flow in composite porous media, Geophys. Res. Lett., 27(12), 1759-1762.

Winter, C. L., D. M. Tartakovsky, and A. Guadagnini (2002), Numerical solutions of moment equations for flow in heterogeneous composite aquifers, Water Resour. Res., 38(5), 1055, doi:10.1029/2001WR000222.

Winter, C. L., D. M. Tartakovsky, and A. Guadagnini (2003), Moment differential equations for flow in highly heterogeneous porous media, Surv. Geophys., 24(1), 81-106.

Wu, X. H., Y. Efendiev, and T. Y. Hou (2002), Analysis of upscaling absolute permeability, Discrete Contin. Dyn. Syst., Ser. B, 2(2), 185-204.

Zijl, W., and J. M. T. Stam (1992), Modeling permeability in imperfectly layered porous-media. 1. Derivation of block-scale permeability tensor for thin grid-blocks, Math. Geol., 24(8), $865-883$.

Zinn, B., and C. F. Harvey (2003), When good statistical models of aquifer heterogeneity go bad: A comparison of flow, dispersion, and mass transfer in connected and multivariate Gaussian hydraulic conductivity fields, Water Resour. Res., 39(3), 1051, doi:10.1029/2001WR001146.

Zlotnik, V. A., and B. R. Zurbuchen (2003), Field study of hydraulic conductivity in a heterogeneous aquifer: Comparison of single-borehole measurements using different instruments, Water Resour. Res., 39(4), 1101, doi:10.1029/2002WR001415.

J. Carrera and X. Sanchez-Vila, Department of Geotechnical Engineering and Geosciences, Technical University of Catalonia, Gran Capità S/N, 08034 Barcelona, Spain.

A. Guadagnini, Dipartimento di Ingegneria Idraulica, Ambientale, Infrastrutture Viarie, Rilevamento, Politecnico di Milano, Piazza L. Da Vinci 32, 20133 Milano, Italy. (alberto.guadagnini@polimi.it) 